Supporting Information for

\title{
Mapping the Reactivity of Dicobalt Bridging Nitrides in Constrained Geometries
}

\author{
Ariana Z. Spentzos and Neil C. Tomson
}

Roy and Diana Vagelos Laboratories, Department of Chemistry, University of Pennsylvania, Philadelphia, Pennsylvania, 19104, United States

\section{Table of Contents}



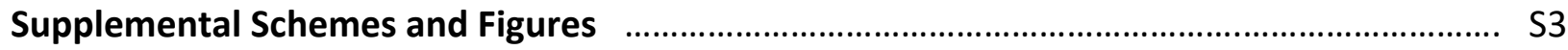

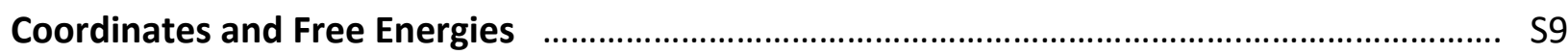




\section{General Considerations}

All density functional theory (DFT) calculations were performed with the ORCA program package, v3.0.3. ${ }^{1}$ Geometry optimizations were carried out at the B97-D3 level of DFT, ${ }^{2}$ using model compounds derived from crystallographic data and truncated at the para- ${ }^{t} \mathrm{Bu}$ positions. This functional was chosen due to its reliable ability to predict bond lengths and angles within this system to within $2 \%$ of their crystallographically determined values. The def2-TZVP basis sets and the def2-TZVP/J auxiliary basis sets (used to expand the electron density in the resolution-of-identity (RI) approach) were used for $\mathrm{Co}, \mathrm{Cl}, \mathrm{P}$, and $\mathrm{N}^{3}$ All other atoms were described using the def2-SV(P) basis sets and def2-SV/J auxiliary basis sets. The Conductor-like Screening Model (COSMO; dipole moment corresponding to acetonitrile) was implemented throughout.

The SCF calculations were tightly converged $\left(1 \times 10^{-8} \mathrm{E}_{\mathrm{h}}\right.$ in energy, $1 \times 10^{-7} \mathrm{E}_{\mathrm{h}}$ in the density change, and $5 \times 10^{-7}$ in the maximum element of the DIIS error vector). In all cases the geometries were considered converged after the energy change was less than $1 \times 10^{-6} \mathrm{E}_{\mathrm{h}}$, the gradient norm and maximum gradient element were smaller than $3 \times 10^{-4} \mathrm{E}_{\mathrm{h}}-\mathrm{Bohr}^{-1}$ and $1 \times 10^{-4} \mathrm{Eh}_{\mathrm{h}}-\mathrm{Bohr}^{-1}$, respectively, and the root-mean square and maximum displacements of all atoms were smaller than $6 \times 10^{-4}$ Bohr and $1 \times 10^{-3}$ Bohr, respectively. All singlets were found to be pure with respect to spin-state contamination under the unrestricted Kohn-Sham approach. Numerical frequency calculations were used to verify that the calculated structures represented either local minima (ground states) or saddle points (transition states) on the potential energy surface. The reported energies are Gibbs free energies at $298.15 \mathrm{~K}$ and $1.00 \mathrm{~atm}$, as obtained from numerical frequency calculations on the optimized geometries.

Transition state searches were performed by using relaxed coordinate scans, involving successive geometry optimization calculations on species related by a regular change to a bond distance of interest. Doing so provided suitable starting points for performing dedicated transition state optimization calculations.

1) F. Neese, WIREs Comput. Mol. Sci. 2012, 2, 73-78.

2) a) S. Grimme, J. Comput. Chem. 2006, 27, 1787-1799; b) S. Grimme, J. Antony, S. Ehrlich, H. Krieg, J. Chem. Phys. 2010, 132, 154104; c) S. Grimme, S. Ehrlich, L. Goerigk, J. Comput. Chem. 2011, 32, 1456-1465.

3) a) A. Schäfer, H. Horn, R. Ahlrichs, J. Chem. Phys. 1992, 97, 2571-2577; b) A. Schäfer, C. Huber, R. Ahlrichs, J. Chem. Phys. 1994, 100, 5829-5835; c) F. Weigend, R. Ahlrichs, Phys. Chem. Chem. Phys. 2005, 7, 3297-3305. 


\section{Supplemental Schemes and Figures}



Scheme S1. Conversion of ${ }^{\mathrm{n}}\left[\mathrm{CO}_{2} \mathrm{~N}_{3}\right]^{3+}$ to form ${ }^{\mathrm{n}}\left[\mathrm{CO}_{2} \mathrm{~N}\right]^{3+}$ with loss of $\mathrm{PMe}_{3}$ preceding $\mathrm{N}_{2}$ dissociation. 


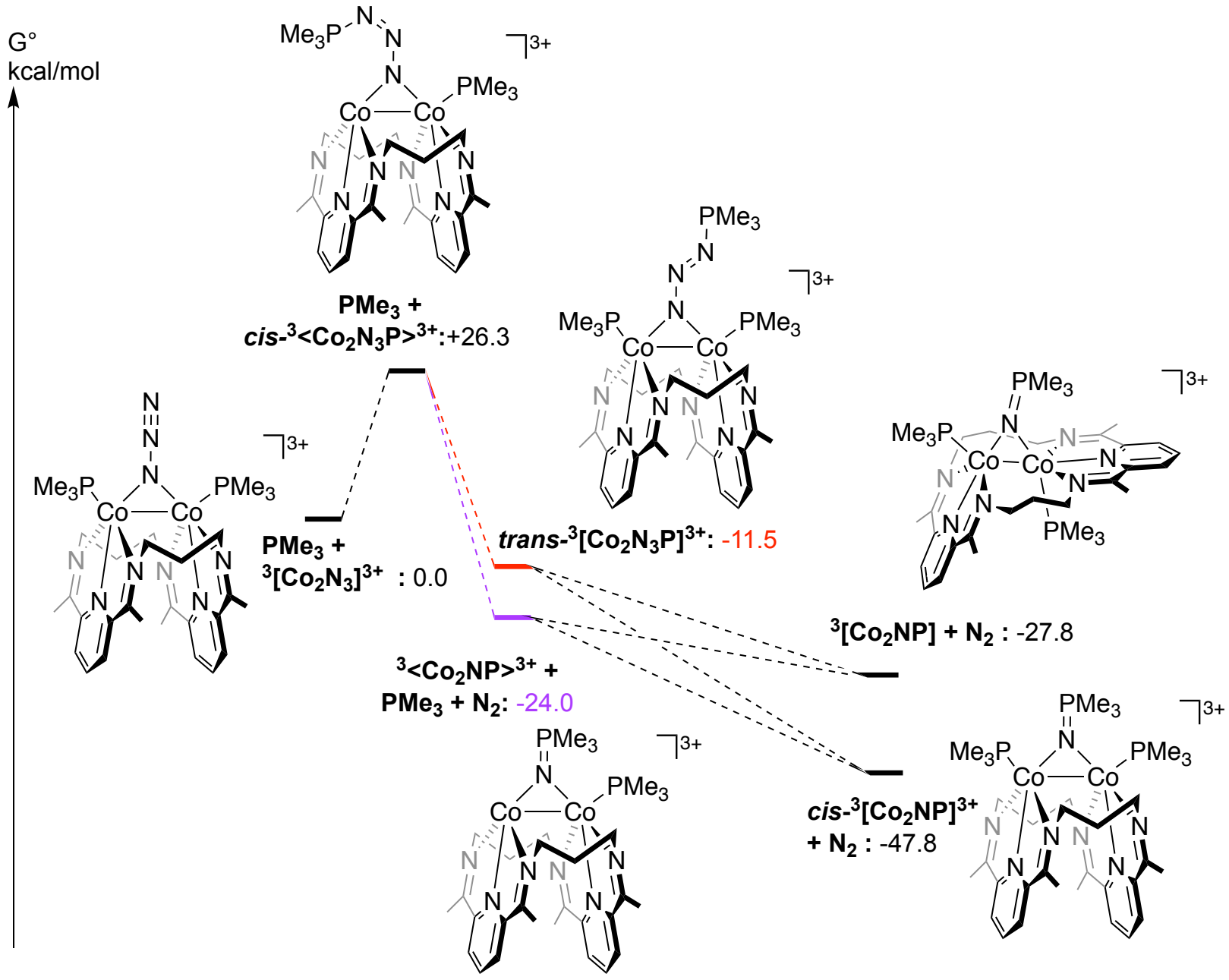

Scheme S2. Intermediates for a metallo-Staudinger pathway from ${ }^{3}\left[\mathrm{Co}_{2} \mathbf{N}_{3}\right]^{3+}$ to ${ }^{3}\left[\mathrm{Co}_{2} \mathbf{N P}\right]^{3+}$. 


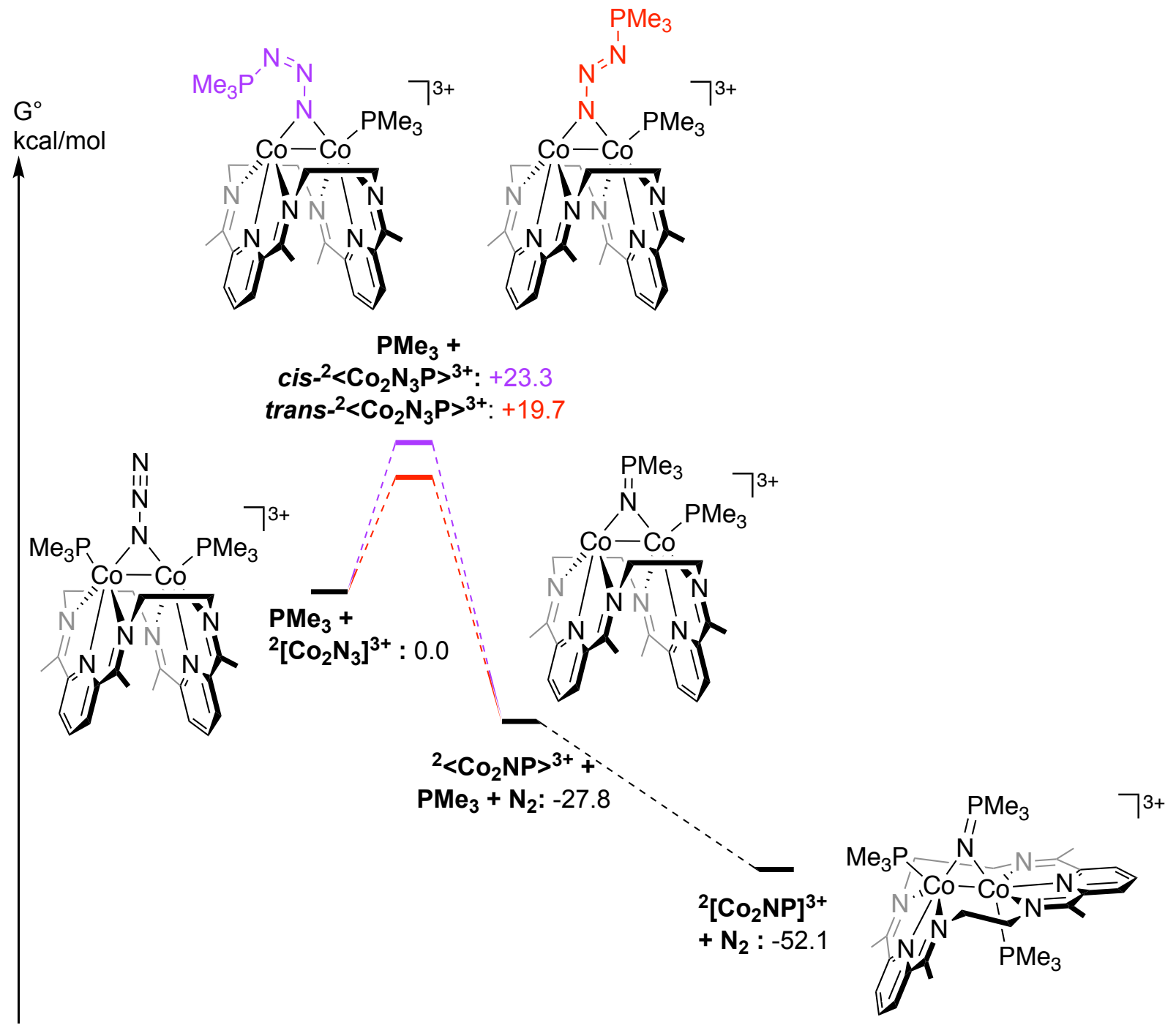

Scheme S3. Intermediates for a metallo-Staudinger pathway from ${ }^{2}\left[\mathrm{Co}_{2} \mathrm{~N}_{3}\right]^{3+}$ to ${ }^{2}\left[\mathrm{Co}_{2} \mathrm{NP}\right]^{3+}$. 

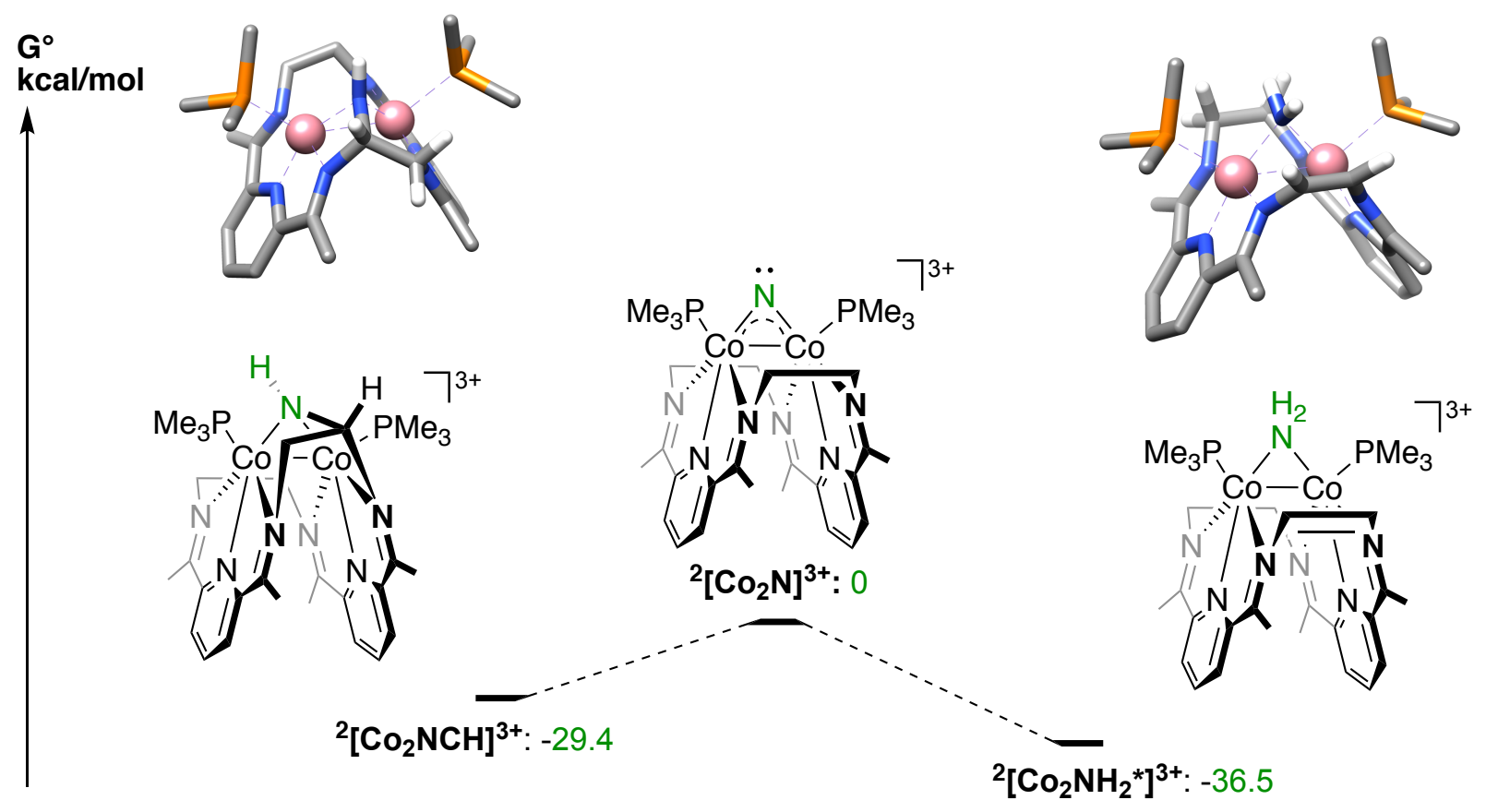

Scheme S4. Optimized structures (left and right) from attempts to locate a $\mu$-NH intermediate following PCET on the singlet energy surface. On left: ${ }^{2}\left[\mathrm{CO}_{2} \mathrm{NCH}\right]^{3+}$ insertion product and right: ${ }^{2}\left[\mathrm{CO}_{2} \mathrm{NH}_{2}{ }^{*}\right]^{3+}$ a bridging amide with a dehydrogenated $\left(\mathrm{C}_{2} \mathrm{H}_{2}\right)$ linker.

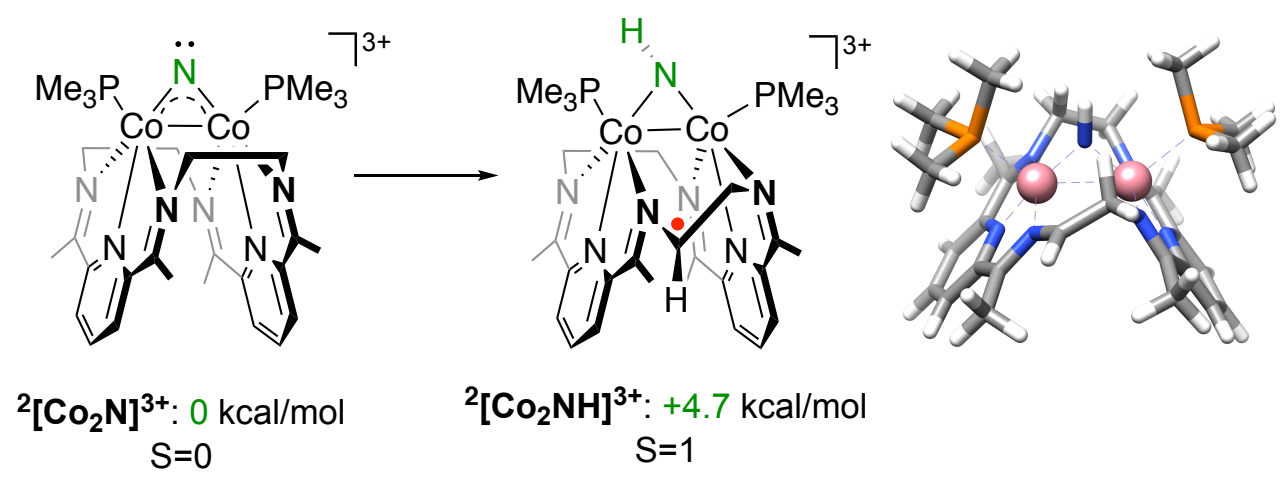

Scheme S5. A $\mu$-NH intermediate located on the triplet surface en route to formation of ${ }^{2}\left[\mathrm{CO}_{2} \mathrm{NH}_{2}\right]^{3+}$. 


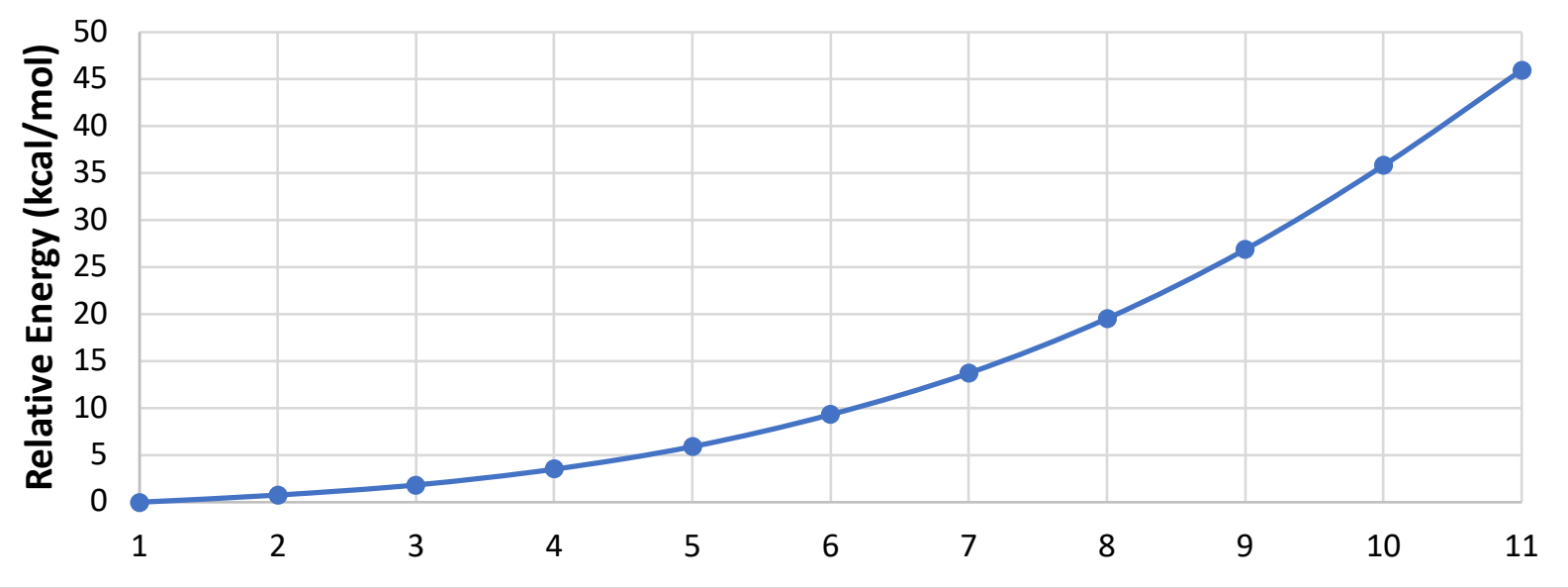

Figure S1. Data from a relaxed coordinate scan of the $\mu-\mathrm{N}_{\mathrm{N} 3}-\mathrm{HC}_{\beta}$ distance from $2.10 \AA$ (point 1 ) to $1.10 \AA$ (point 11$)$ in $\left[\left({ }^{2} \mathrm{PDI}_{2}\right) \mathrm{Co}_{2}\left(\mu-\mathrm{N}_{3}\right)\left(\mathrm{PMe}_{3}\right)_{2}\right]^{3+}$. The $\mathrm{N}-\mathrm{N}$ distance varied from $1.21-1.27 \AA$.
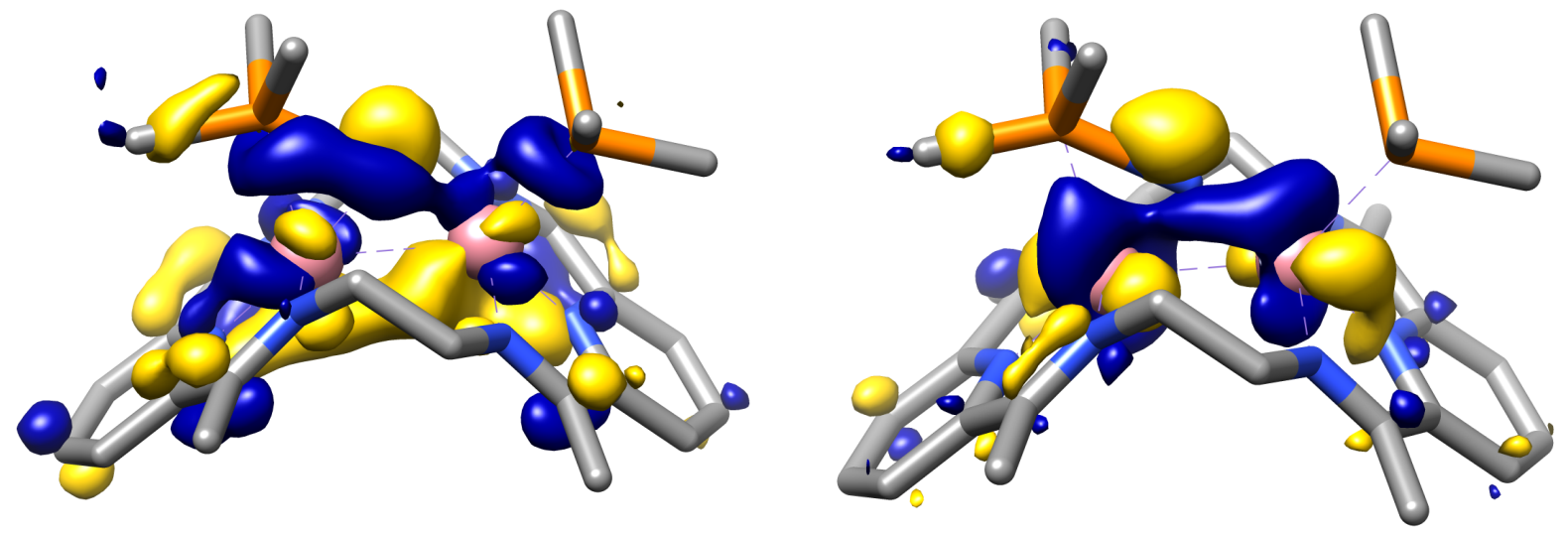

Figure S2. HOMO (left) and HOMO-2 (right) of ${ }^{2} \mathrm{TS}_{\mathrm{NP}}$. 

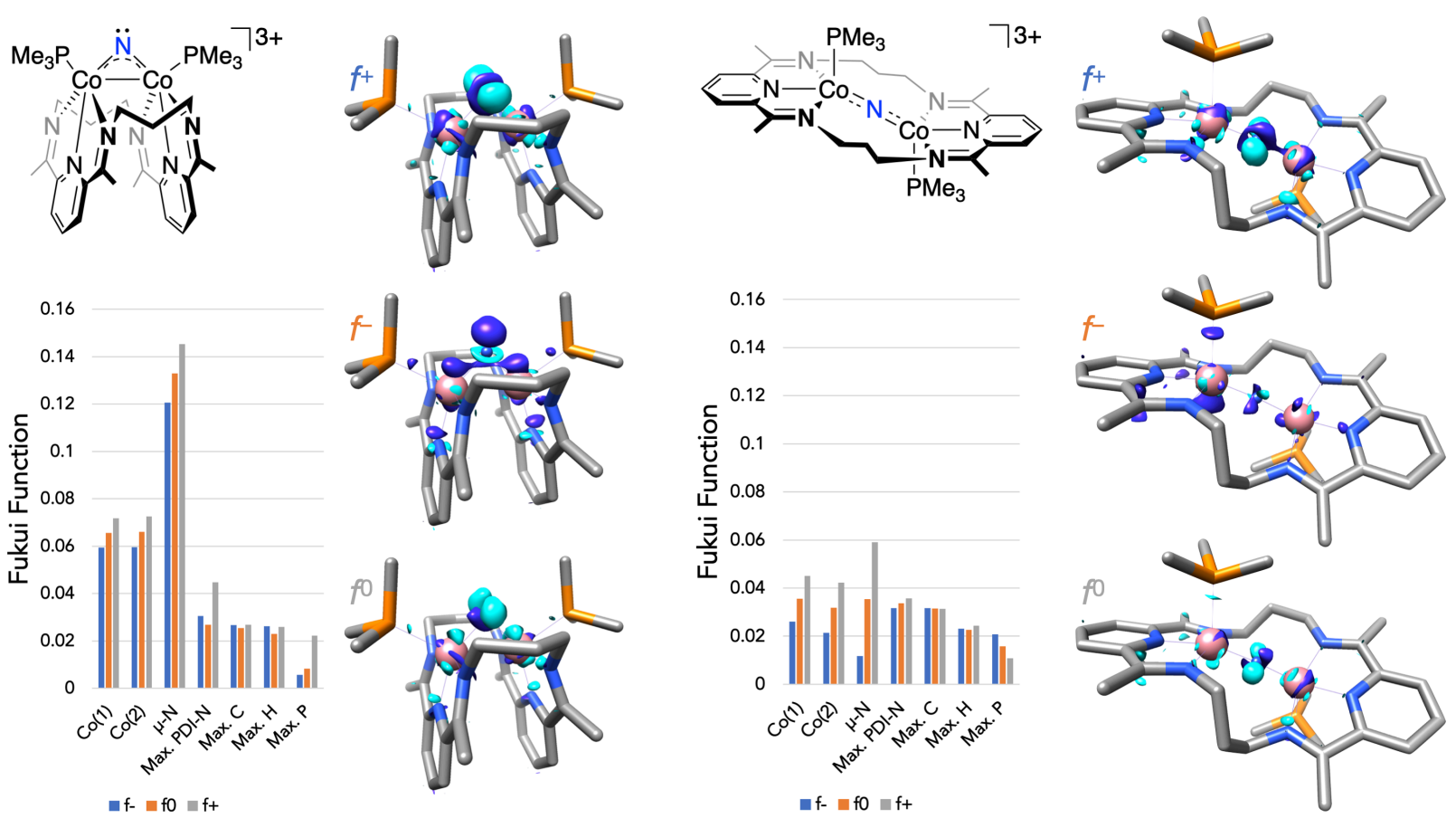

Figure S3. Fukui function analyses of ${ }^{3}\left[\mathrm{CO}_{2} \mathrm{~N}\right]^{3+}$ and $\operatorname{lin}^{-}\left[\mathrm{CO}_{2} \mathrm{~N}\right]^{3+}$. Bar graphs display condensed (Mulliken) Fukui function data, and molecular structure plots show the full electron density difference maps.


Figure S4. Fukui function analyses of ${ }^{2}\left[\mathrm{CO}_{2} \mathrm{~N}\right]^{3+}$ and $\operatorname{lin}^{2}\left[\mathrm{Co}_{2} \mathrm{~N}\right]^{3+}$. Bar graphs display condensed (Mulliken) Fukui function data, and molecular structure plots show the full electron density difference maps. 

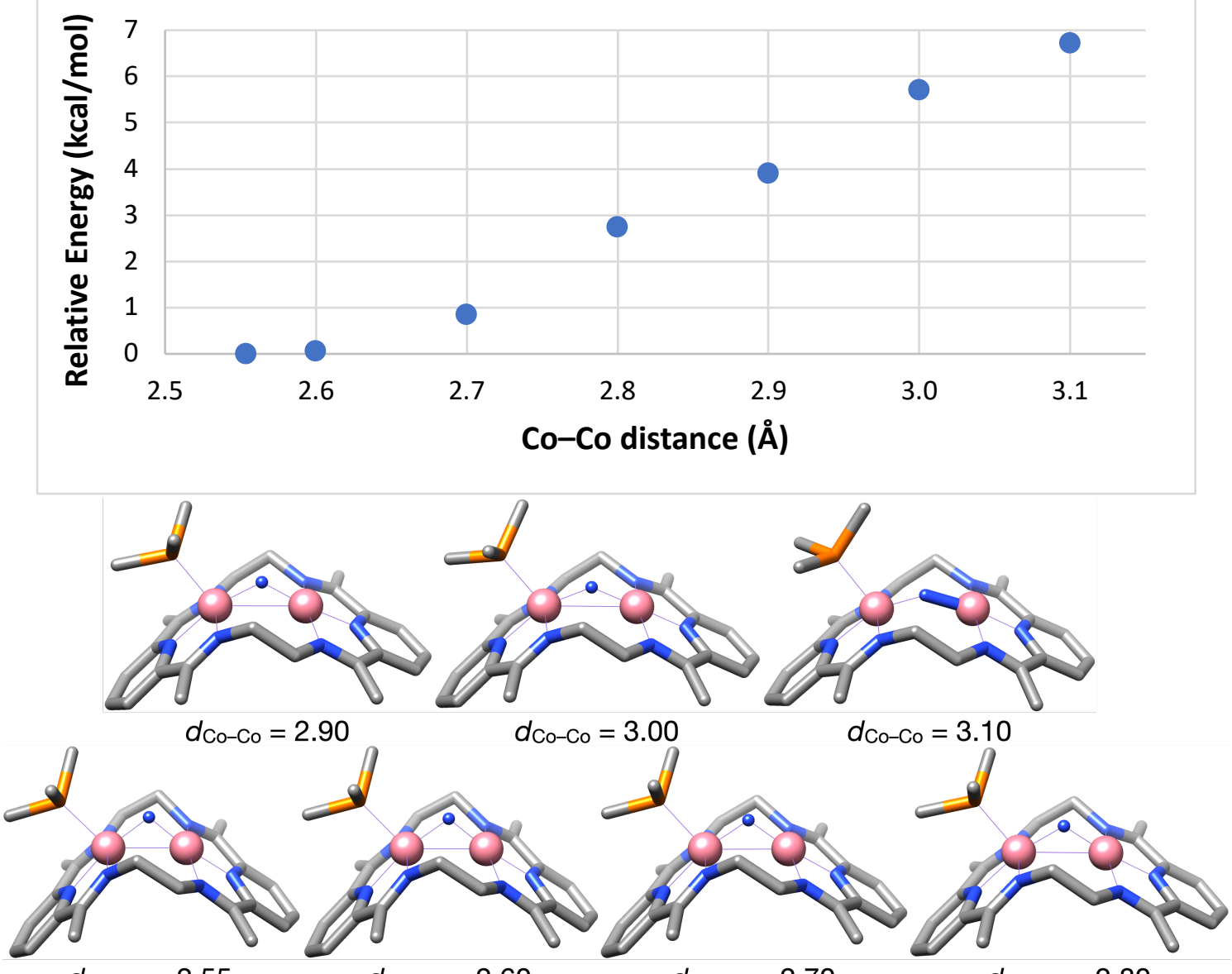

$d_{\mathrm{Co}-\mathrm{Co}}=2.55$

$d_{\mathrm{Co}-\mathrm{Co}}=2.60$

$d_{\mathrm{Co}-\mathrm{Co}}=2.70$

$d_{\mathrm{Co}-\mathrm{Co}}=2.80$

Figure S5. Data from a relaxed coordinate scan of the $\mathrm{Co}-\mathrm{Co}$ distance in $\left[\left({ }^{2} \mathrm{PDI}_{2}\right) \mathrm{Co}_{2}(\mu-\mathrm{N})\left(\mathrm{PMe}_{3}\right)\right]^{3+}$, starting from the optimized geometry of ${ }^{2}\left\langle\mathrm{Co}_{2} \mathrm{~N}\right\rangle^{3+}\left(d_{\mathrm{Co}-\mathrm{Co}_{0}}=2.55 \AA\right)$.

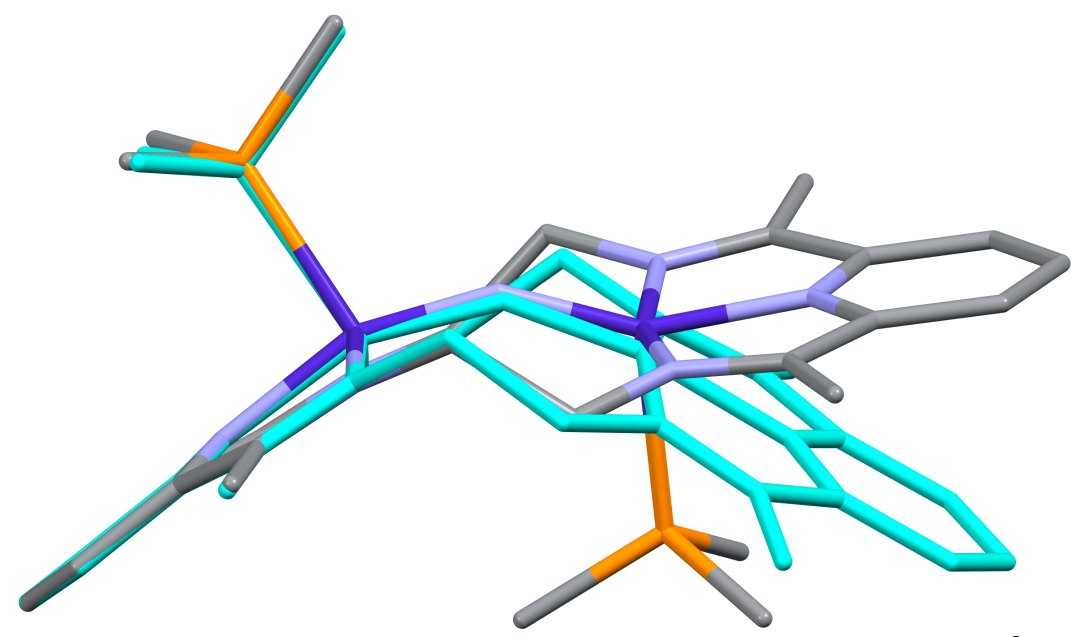

Figure S6. Overlay of the optimized geometry of $/ \operatorname{lin}^{-2}\left[\mathrm{Co}_{2} \mathrm{~N}\right]^{3+}\left(d_{\mathrm{Co}-\mathrm{Co}_{0}}=3.11 \AA \AA\right)$ in element colors and the $d_{\mathrm{Co}_{0} \mathrm{C}_{0}}=3.10 \AA$ A structure of $\left[\left({ }^{2} \mathrm{PDI}_{2}\right) \mathrm{Co}_{2}(\mu-\mathrm{N})\left(\mathrm{PMe}_{3}\right)\right]^{3+}$ in cyan, taken from the relaxed coordinate scan shown in Figure S3. 


\section{Coordinates and Free Energies}

\begin{tabular}{|c|c|c|c|}
\hline \multicolumn{4}{|c|}{$\left[\left({ }^{3} \mathrm{PDI}_{2}\right) \mathrm{Co}_{2}(\mu-\mathrm{Cl})\left(\mathrm{PMe}_{3}\right)_{2}\right]^{3+}\left({ }^{3}\left[\mathrm{Co}_{2} \mathrm{Cl}\right]^{3+}, \mathrm{S}=0\right)$} \\
\hline & -0.03006924961157 & 0.00767266820470 & -0.03393953158344 \\
\hline & -0.28545209125097 & -0.46130534992272 & -2.59099383968038 \\
\hline & -0.21698474290677 & -2.04355279627853 & -0.97152936812805 \\
\hline & 0.08800138652775 & -0.35584186589554 & 2.18618276892024 \\
\hline & -0.50551062165417 & -1.60358250977589 & -4.51709061473202 \\
\hline & 1.89614112719585 & 0.18285221641258 & -0.14007269655971 \\
\hline & 0.05301541576767 & 1.81773244403877 & -0.08058027403116 \\
\hline & -1.92419000244337 & 0.36621343697687 & 0.17015471431645 \\
\hline & -2.19918666574087 & -0.13174776354837 & -2.57577003717903 \\
\hline & -0.25163152296110 & 1.23219844325121 & -3.23540896753363 \\
\hline & 1.62310969871199 & -0.33240492448880 & -2.89414901801753 \\
\hline & 2.34316730833395 & 1.41939620796179 & -0.14640012640913 \\
\hline C & 1.27345015983442 & 2.41135784477273 & -0.16717341759697 \\
\hline & 1.35883521899663 & 3.80888639264602 & -0.24032550247595 \\
\hline & 2.33408096396560 & 4.29988047365742 & -0.32 \\
\hline C & 0.17487999677426 & 4.56531080665482 & -0.203760 \\
\hline & -1.06652496110397 & 2017 & 349 \\
\hline $\mathrm{H}$ & -1.99285804218261 & 438 & 335 \\
\hline & -1.10363859552821 & 2.52547773 & 0.0205983 \\
\hline & -2.24511609914677 & 1.64109467465950 & 6812 \\
\hline & -2.94162621172333 & -0.681787 & 6264 \\
\hline $\mathrm{H}$ & -3.52045579439878 & 0066 & 954 \\
\hline $\mathrm{H}$ & -2.41239068934627 & -1.63 & 0.4 \\
\hline C & -3.89273171312085 & 4424 & -0.8 \\
\hline $\mathrm{H}$ & -4.60813062139011 & -1.61 & -0. \\
\hline $\mathrm{H}$ & -4.48626966476116 & 62544 & -0.9 \\
\hline C & -3.19923695111775 & -1.135 & -2.1 \\
\hline $\mathrm{H}$ & -2.677623 & -2.09 & -2. \\
\hline $\mathrm{H}$ & -3.96210844638460 & -1.24 & -2.96 \\
\hline C & -2.56159281808988 & 1.04 & -3.04 \\
\hline & -1.428537 & 1.89 & -3.3 \\
\hline C & -1.42197096604833 & 3.21 & -3.85 \\
\hline & -2.36133 & 3.76 & -3.9 \\
\hline C & -0.1924514 & 3.82 & -4.1 \\
\hline C & 1.00218018652957 & 3.089 & -4.056 \\
\hline $\mathrm{H}$ & 1.96267134202587 & 3.538 & -4.329602 \\
\hline C & 0.94718826584858 & 1.769 & -3.58 \\
\hline C & 2.02628269110886 & 0.80167798453272 & -3.42295074960765 \\
\hline C & 2.56434589841427 & -1.43962408165329 & -2.65914706389298 \\
\hline $\mathrm{H}$ & 3.16409902114703 & -1.61556820220013 & -3.570885 \\
\hline $\mathrm{H}$ & 1.96940706249192 & -2.34414247045051 & -2.47027009824851 \\
\hline C & 3.51132206059284 & -1.18287256736835 & -1.48332143789601 \\
\hline $\mathrm{H}$ & 4.16280827146953 & -2.07208042169859 & -1.38093735507382 \\
\hline $\mathrm{H}$ & 4.17337471633099 & -0.32951159909527 & -1.71459340602068 \\
\hline C & 2.82111431545892 & -0.96149588056578 & -0.13430842622792 \\
\hline $\mathrm{H}$ & 2.23998239075828 & -1.85312627279370 & 0.14322995514717 \\
\hline $\mathrm{H}$ & 3.59439791521553 & -0.80895676432647 & 0.64014483875976 \\
\hline & 3.77585823939732 & 1.85196848283445 & -0.08079830784326 \\
\hline $\mathrm{H}$ & 4.46289212570492 & 1.03133560713092 & 0.17752485293383 \\
\hline & 3.88702994141250 & 2.65114817891782 & 0.67774336503669 \\
\hline & 4.10317531076759 & 2.28521780530351 & -1.04712670634398 \\
\hline & -3.59810364374980 & 2.20871653721608 & 0.52649719232245 \\
\hline $\mathrm{H}$ & -4.29027084146942 & 1.4629 & $0.947 \varepsilon$ \\
\hline $\mathrm{H}$ & -4.06298391594234 & 2.635 & -0.384940 \\
\hline $\mathrm{H}$ & -3.49617218275939 & 3.03971367610300 & 1.25070831276486 \\
\hline C & -3.95879204796778 & 1.51693942541154 & -3.29982072900624 \\
\hline $\mathrm{H}$ & -4.72229628469076 & 0.74019838569436 & -3.14173930835230 \\
\hline $\mathrm{H}$ & -4.03419371761092 & 1.87029220794310 & -4.34692121865120 \\
\hline $\mathrm{H}$ & -4.20694112216521 & 3245767 & -2.65637617311199 \\
\hline C & 3.40802146972808 & 1.12494788408432 & -3.90236329269430 \\
\hline
\end{tabular}




$\begin{array}{llll}\text { H } & 4.06383102701878 & 0.24210407050371 & -3.95909847857105 \\ \text { H } & 3.89150851785530 & 1.87241519979787 & -3.24197125627963 \\ \text { H } & 3.34988909706570 & 1.58096676303531 & -4.90929573296797 \\ \text { C } & 1.73857166367152 & -0.14597984918479 & 2.92582497618053 \\ \text { H } & 1.63980195313126 & -0.20334325075352 & 4.02744158314683 \\ \text { H } & 2.15221983326505 & 0.84102593463399 & 2.65047953579864 \\ \text { H } & 2.41909997834964 & -0.94397920061392 & 2.58420778072175 \\ \text { C } & -0.44284424469876 & -2.01406817102463 & 2.71047391921621 \\ \text { H } & -0.30775154741217 & -2.10764726647742 & 3.80596928097518 \\ \text { H } & 0.17042868136220 & -2.77358142164914 & 2.19313018166996 \\ \text { H } & -1.50530357767777 & -2.17023046687992 & 2.45812455489995 \\ \text { C } & -0.94334575042361 & 0.81218177855897 & 3.12727730073329 \\ \text { H } & -2.01235993960646 & 0.64851184076638 & 2.90591337267050 \\ \text { H } & -0.66939781572583 & 1.85019077837388 & 2.86378740177992 \\ \text { H } & -0.77012345822796 & 0.65462414558088 & 4.20953329039105 \\ \text { C } & 0.83039486726581 & -1.28636996064408 & -5.71076649862060 \\ \text { H } & 0.57240270837088 & -1.76949819865869 & -6.67197852724654 \\ \text { H } & 1.78488285544210 & -1.69970147999115 & -5.34680600427732 \\ \text { H } & 0.93527003273566 & -0.19819156482861 & -5.87145682095071 \\ \text { C } & -0.55764671396508 & -3.40679924055541 & -4.29493115314850 \\ \text { H } & -0.66207868270125 & -3.89896647041265 & -5.28114823453856 \\ \text { H } & -1.41630001869647 & -3.67323735623663 & -3.65240895149288 \\ \text { H } & 0.37487589257879 & -3.74588940974508 & -3.80945247383347 \\ \text { C } & -2.00443187534382 & -1.18329953878930 & -5.46047189417427 \\ \text { H } & -2.06027292996522 & -0.08999912782572 & -5.61211001654669 \\ \text { H } & -2.90640539870160 & -1.53233111981973 & -4.93118793239635 \\ \text { H } & -1.95250777425114 & -1.68041492894718 & -6.44727471175431 \\ \text { H } & -0.16713477366200 & 4.85668752506747 & -4.52547904266094 \\ \text { H } & 0.22239975684223 & 5.65821200306028 & -0.27395049124336\end{array}$

$\begin{array}{cccc}{\left[\left({ }^{2} \mathrm{PDI}_{2}\right) \mathrm{Co}_{2}(\mu-\mathrm{Cl})(\mathrm{PMe})_{2}\right]^{3+}\left({ }^{2}\left[\mathrm{Co}_{2} \mathrm{Cl}\right]^{3+}, \mathrm{S}=0\right)} \\ \mathrm{E}=-5329.37368112 & E_{\mathrm{h}} & \\ \mathrm{Co} & -0.00721163608145 & 0.02812447965272 & 0.03220700091608 \\ \mathrm{Co} & -0.00328907319729 & 0.03725481456995 & 2.56710779380198 \\ \mathrm{Cl} & -0.34104029650613 & 1.87295030202352 & 1.29344023110722 \\ \mathrm{P} & -0.17693812872288 & 0.91354856564418 & -2.01489785620092 \\ \mathrm{P} & -0.17936761828177 & 0.93876983675916 & 4.60769257628877 \\ \mathrm{~N} & 1.91392772819363 & 0.11069040614414 & 0.01435694795692 \\ \mathrm{~N} & 0.31495873641747 & -1.69647291540472 & -0.41006588617006 \\ \mathrm{~N} & -1.84347009325233 & -0.58716821131094 & 0.00929807536403 \\ \mathrm{~N} & -1.83969703467876 & -0.57275986578708 & 2.58570677614518 \\ \mathrm{~N} & 0.30783302148963 & -1.68299545117717 & 3.03109503147214 \\ \mathrm{~N} & 1.93171506904860 & 0.11390598493439 & 2.59752751513723 \\ \mathrm{C} & 2.53038108985550 & -0.99765701053233 & -0.34313966454716 \\ \mathrm{C} & 1.60423085303815 & -2.08988249527084 & -0.61520494617291 \\ \mathrm{C} & 1.86643084585539 & -3.40881515735942 & -1.01572548246493 \\ \mathrm{H} & 2.89453903036724 & -3.74417432390190 & -1.18274514613373 \\ \mathrm{C} & 0.79157933188004 & -4.29330162334797 & -1.20243050362103 \\ \mathrm{C} & -0.52683981884408 & -3.85304868685783 & -1.00127934545627 \\ \mathrm{H} & -1.37384793458867 & -4.52916136786385 & -1.15393576846970 \\ \mathrm{C} & -0.74493921908939 & -2.52676272070810 & -0.60283227812452 \\ \mathrm{C} & -2.00797179079432 & -1.84914747518666 & -0.32815332764493 \\ \mathrm{C} & -2.94382559108610 & 0.23201236901575 & 0.51080160595796 \\ \mathrm{H} & -3.91293867537660 & -0.12983975403771 & 0.13650641685402 \\ \mathrm{H} & -2.79961342270146 & 1.25756498535046 & 0.14809443434241 \\ \mathrm{C} & -2.93806277873501 & 0.24516121016468 & 2.07815220804053 \\ \mathrm{H} & -2.78334634031954 & 1.27538109863311 & 2.42250043035908 \\ \mathrm{H} & -3.90667792010826 & -0.10236424362217 & 2.46839171041600 \\ \mathrm{C} & -2.01187341173335 & -1.83213189713366 & 2.92913159401374 \\ \mathrm{C} & -0.75434751559460 & -2.51060385789655 & 3.22409757696189 \\ \mathrm{C} & -0.53817570177848 & -3.83113830681174 & 3.64412416065410 \\ \mathrm{H} & -1.38583271444156 & -4.50566892934032 & 3.79996084369191 \\ \mathrm{C} & 0.77811125560835 & -4.26859540801198 & 3.86606323696634 \\ \mathrm{C} & 1.85444347416130 & -3.38621827536360 & 3.67567943919400 \\ \mathrm{H} & 2.88416334434854 & -3.71147539992912 & 3.85544930042893\end{array}$


C 1.59085969696681

C 2.53127409127798

C 2.67183713811370

H 3.70590252717509

H 2.17423791198332

C 2.66656298656019

H 2.17727091681251

H 3.69808836385441

C 4.01598831030452

H 4.38587146534535

H 4.29722511822847

H 4.55310481163354

C -3.29791763460684

H -4.17399073427046

H -3.23907450623192

H -3.46906740048101

C -3.31067247378076

H -3.56970278568175

H -3.21362628379198

H -4.15152498875861

C 3.99971210161279

H 4.62607327975273

H 4.31851983930491

H 4.20531628770471

C -1.75882401905199

H -1.72579777097178

H -1.91660552214954

H -2.58867189474062

C -0.00799318790277

H -0.09783469218243

H -0.80207585497315

H 0.97995126623665

C 1.08650016402253

H 2.09289868684708

H 1.00965160073700

H 0.91676724199207

C -1.77085476311168

H -2.59155579291270

H -1.94067638027653

H -1.74029965778562

C 0.01093091735289

H -0.09100724565028

H 1.00888961703292

H -0.76735788536067

C 1.06793609604199

H 0.89812903522859

H 0.97445052309068

H 2.08079196348291

H 0.96505390248638

H 0.98254854913859
$-2.07561230850945$

$-0.99443796534122$

1.26416067087335

1.27188065884425

2.17579637127731

1.25866326426230

2.17392721169820

1.24927091996127

$-1.14672455688799$

$-0.53615317219770$

$-2.19470143046929$

$-0.80970211845615$

$-2.59951187798821$

$-2.04192388555868$

$-3.55444882547946$

$-2.85786544050378$

$-2.56827553780977$

$-2.68528644599584$

$-3.58549949152969$

$-2.06701980783746$

$-1.22860807171601$

$-0.36889397533075$

$-2.10726381575655$

$-1.47404940723246$

1.73378938574050

2.13573455550992

2.56300947759763

1.01002068144088

$-0.37680245652229$

0.07758309996915

$-1.13521422225569$

$-0.86351358758844$

2.15337743602181

1.70475802178641

3.00976581010813

2.50693280210827

1.74195554026183

1.00707597811654

2.56221348600621

2.15490777473048

$-0.33493662627034$

0.12850835394945

$-0.80309412556466$

$-1.11009470079274$

2.20004013693869

2.55884023984053

3.04995171143261

1.76658964792478

$-5.29618953959264$

$-5.32585051924965$
3.25339541132697

2.97824443317574

2.08228186722812

2.45706913295974

2.43574095931742

0.51418997317438

0.15823352384252

0.13186306151935

$-0.47582067674643$

$-1.32475859943249$

$-0.66651687232190$

0.42802243255458

$-0.45174268846701$

$-0.08997583016418$

0.10255329428077

$-1.51641815278798$

3.04689356747905

4.11919001205477

2.62616607398643

2.54519901147445

3.14920122789669

2.86964149168203

2.55587612933456

4.21081413952052

$-2.37270647168962$

$-3.40367054213644$

$-1.66084609781461$

$-2.29238298893828$

$-3.28260785867683$

$-4.28786618027570$

$-3.15524421114788$

$-3.19177237567950$

$-2.42209671357526$

$-2.35303707892176$

$-1.72889383970366$

$-3.45733322621840$

4.96115050875179

4.89016457085244

4.24170372200850

5.98786440401234

5.88931872033285

6.88926240461747

5.80849672823138

5.76699268076684

4.99897855944437

6.03243514936107

4.29989141440750

4.92829308579733

4.19619401043011

$-1.51442599818843$

$\left[\left({ }^{3} \mathrm{PDI}_{2}\right) \mathrm{Co}_{2}\left(\mu-\mathrm{N}_{3}\right)\left(\mathrm{PMe}_{3}\right)_{2}\right]^{3+}\left({ }^{3}\left[\mathrm{Co}_{2} \mathbf{N}_{3}\right]^{3+}, \mathrm{S}=0\right)$

$\mathrm{E}=-5111.74625539 E_{\mathrm{h}}$

Co $-0.01907137529585 \quad-0.07748700513774 \quad-0.01158509924251$

Co $0.00236403060853 \quad-0.04583629476902 \quad 2.54515405265253$

$\begin{array}{llll}\text { P } & 0.01983996807078 & -0.94027458280276 & -2.09141494626908\end{array}$

$\begin{array}{lllll}\text { P } & 0.05331077570632 & -0.85824840677674 & 4.64502470347415\end{array}$

N $-1.94428856764930 \quad 0.07667820859196 \quad-0.12615157490868$

N $-0.10575684837431 \quad 1.69810121272203 \quad-0.38093105684091$

N $1.88150508442710 \quad 0.26526611019724 \quad-0.13747162215192$

N $1.90381633770796 \quad 0.30169235479539 \quad 2.65616316179466$

$\begin{array}{llll}N & -0.08694093568193 & 1.73533823295181 & 2.88740907456401\end{array}$

N $-1.92399460909896 \quad 0.10712734019370 \quad 2.66729132280598$

N $-0.00423712745791 \quad-1.54488777323937 \quad 1.28617678495657$

N $0.46975382585706 \quad-2.66085898613074 \quad 1.29836715162913$ 
N 0.88025443782073

C -2.39343480389342

C -1.33035890697542

C -1.42536363900006

H -2.40507425828161

C -0.24333308465380

C 1.00878001382436

H 1.93445988040974

C 1.05442986007074

C 2.21039066517960

C 2.89779797240532

H 3.57719047095955

H 2.37783855265751

C 3.73116703612681

H 4.43109687690273

H 4.35266103708332

C 2.92185564873778

H 2.40405446273502

H 3.61679419151678

C 2.22971873452259

C 1.07210954265044

C 1.02798603114248

H 1.95456155777056

C -0.22410922746166

C -1.40592232142852

H -2.38528203203889

C -1.31156328710841

C -2.37383541040477

C -2.82214543673787

H -3.49313147343278

H -2.19116980342417

C -3.66540923093554

H -4.37213458792583

H -4.27934208745495

C -2.84409314499607

H -2.21617955871075

H -3.52827585001368

C -3.81121950127221

H -3.84714609966553

H -4.20869336603094

H -4.48638131711206

C 3.58823744460571

H 3.95999639150030

H 3.56631039270528

H 4.31986375480245

C 3.60029894741302

H 4.37273708319878

H 3.58711948136409

H 3.90265192095824

C -3.79632966108689

H -4.21809187196400

H -3.84234149015047

H -4.44806256361882

C -1.43349767838346

H -1.56900465474918

H -2.34183928222411

H -1.26394477097212

C 0.13099634636168

H -0.72348139893368

H 1.07359649224112

H 0.11440830931035

C 1.40042582337366

H 1.25938283095177

H 2.36443508599312

H 1.40687657125926

C 0.14777854404325
$-3.73223099561844$ 1.27570040159210 2.27298481689311 3.65454575732717 4.13347042398516 4.40987891690710 3.77786174808465

4.35368385577153 2.39298457280172 1.50619910988888 $-0.79016520646659$ $-0.75419786952619$ $-1.75859688038495$ $-0.69876913236811$ $-1.55607866741070$ 0.21416389273681

$-0.75425586385176$ $-1.72185160832215$ $-0.69349396425046$ 1.54629730197556 2.43239408372095 3.81920189421451 4.39463764074329 4.45208638088995 3.69527857470703 4.17592219043459 2.31084353970610 1.31111262259932 $-1.05309432865081$ $-1.08321592157630$ $-1.95402110267058$ $-1.06378823003421$ $-0.21375986838347$ $-1.98456030935676$ $-1.07941570025449$

$-1.98286068648948$

$-1.12173435398496$

1.65561812471985

2.21882173613355

2.33198259984655

0.79016233886055

2.02430101589230

2.62462769703233

2.69749266525908

1.22445943061445

2.07680177236559

1.29505255448889

2.62316271780429

2.80967656621865

1.69152033242724

2.26714966071242

2.35137047243479

0.82192598878897

$-0.50948809842656$

0.58758547103299

$-0.99144835112624$

$-0.85729686891518$

$-2.75494294776248$

$-3.19960161222864$

$-3.08694761359335$

$-3.09389832786508$

$-0.33604655507679$

$-0.69420032773692$

$-0.71151739035991$

0.76971298471212

$-2.67215611442688$
1.30957463245194 $-0.42122567758939$

$-0.51532008358844$

$-0.73552319923016$

$-0.83237255932937$

$-0.82633762030500$

$-0.73672230464967$

$-0.83468812796539$

$-0.51693316993280$

$-0.42406408035772$

$-0.02266653604215$

$-0.89410044962503$

$-0.06484862392040$

1.25654045741207

1.26212054503722

1.23564553050042

2.55370082303436

2.63064098987225

3.41094700549456 2.92860445387714 3.01251551915951

3.21744738005892

3.30853577839212

3.29979133626139

3.22032656114092

3.31295591300305

3.01739697581716

2.94078991132241

2.56829643769104

3.44634835166467

2.59987471103799

1.29238729784597

1.28990871968111

1.30755857008765

0.00227657681817

$-0.02411402392819$

$-0.86475641096987$

$-0.71755195650185$

$-1.67073368803358$

0.06597651430231

$-0.79920110761063$

$-0.69676767114863$

0.15818469505168

$-1.57497454076282$

$-0.89314697323462$

3.21364400628424

3.26963401800453

4.17757730285642

2.43909049857470

3.21304334818834

2.36406874649477

4.10074017366251

3.39242071687840

$-3.10007256554130$

$-3.10528128403004$

$-2.70404442501274$

$-4.13655209000050$

$-2.14361214753588$

$-1.60207115471049$

$-1.67311414328770$

$-3.19755635012674$

$-3.11369037337537$

$-4.15103841330048$

$-2.73422235948060$

$-3.11017411576169$

4.73707997953276 


$\begin{array}{cccc}\text { H } & 1.08207232281981 & -3.02317045917133 & 4.26399364645309 \\ \text { H } & -0.71651202630397 & -3.12147796235742 & 4.21565060960495 \\ \text { H } & 0.14013179609523 & -2.98677395917621 & 5.79862742739093 \\ \text { C } & 1.44827432118629 & -0.24570086376546 & 5.64225934667830 \\ \text { H } & 1.31396488706342 & -0.58391787178911 & 6.68723926470281 \\ \text { H } & 1.46361197460084 & 0.85936495208978 & 5.61829042989930 \\ \text { H } & 2.40534772719351 & -0.63681960836733 & 5.26072977731877 \\ \text { C } & -1.38752449821618 & -0.38893990413752 & 5.65383841352931 \\ \text { H } & -1.21213308073140 & -0.71143126142309 & 6.69761598762617 \\ \text { H } & -2.30363224556442 & -0.87267389560884 & 5.27835256357136 \\ \text { H } & -1.51241238881315 & 0.70904842095079 & 5.63034122180391 \\ \text { H } & -0.27962590737785 & 5.53655101451950 & 3.44686365059193 \\ \text { H } & -0.29834403232808 & 5.49242403043480 & -0.98668054223819\end{array}$

$\left[\left({ }^{2} \mathrm{PDI}_{2}\right) \mathrm{Co}_{2}\left(\mu-\mathrm{N}_{3}\right)\left(\mathrm{PMe}_{3}\right)_{2}\right]^{3+}\left({ }^{2}\left[\mathrm{Co}_{2} \mathrm{~N}_{3}\right]^{3+}, \mathrm{S}=0\right)$

$\mathrm{E}=-5033.27351459 E_{\mathrm{h}}$

Co 0.01081167623008

Co -0.03841313616471

0.01793846227689

$\begin{array}{llll}\text { N }-0.22706600256227 & 1.50659657652565 & 1.31084399106713\end{array}$

$\begin{array}{llll}\text { N } & -0.87707864791904 & 2.53014690779683 & 1.28515449575470\end{array}$

N $-1.45352577024609 \quad 3.52019346254590 \quad 1.26176921541285$

$\begin{array}{llll}\text { P } & -0.09099333664577 & 0.92909443101534 & -1.95946839271431\end{array}$

$\begin{array}{llll}\mathrm{P} & -0.25855224189174 & 1.00971488937345 & 4.60133610844695\end{array}$

$\begin{array}{llll}\text { N } & 1.92754515937123 & 0.10425109394518 & 0.11183451350566\end{array}$

$\begin{array}{llll}\text { N } & 0.34472866842674 & -1.68753631769481 & -0.42862144556023\end{array}$

N $-1.81102197751296 \quad-0.59340541620785 \quad 0.00706578348623$

N $-1.85243951891218 \quad-0.57294941937129 \quad 2.57148459125386$

N $0.27912840753738 \quad-1.64111990747600 \quad 3.14441504941418$

$\begin{array}{llll}\text { N } & 1.88273920818320 & 0.15930455648423 & 2.66847973994508\end{array}$

C $2.55761399792334 \quad-0.99591615562982 \quad-0.23890894937682$

C $1.63931125863619 \quad-2.07892571630908$ - 0.58573694462929

C $1.91485408937072 \quad-3.38723808327017 \quad-1.01153866012903$

H $2.94853216549592 \quad-3.72162473015387 \quad-1.14631261657671$

C $0.84367713271407 \quad-4.26195844453349 \quad-1.26239757174056$

$\begin{array}{llll}\text { C } & -0.48168551081739 & -3.82812223947858 & -1.08840465470475\end{array}$

H $-1.32299676393826 \quad-4.50271675444743 \quad-1.27812435088650$

$\begin{array}{llll}\text { C } & -0.71124310224322 & -2.51180758471442 & -0.66280702782917\end{array}$

C $-1.97812177747858 \quad-1.83921109269724 \quad-0.38080529768566$

$\begin{array}{llll}\text { C } & -2.90838519197015 & 0.24388670274471 & 0.47951415255518\end{array}$

H $-3.87563964738265 \quad-0.09465880598173 \quad 0.07991965753254$

$\begin{array}{llll}\text { H } & -2.74265881241568 & 1.26516274913960 & 0.10532520405441\end{array}$

$\begin{array}{llll}\text { C } & -2.94547027806198 & 0.24077908883074 & 2.04748900939139\end{array}$

$\begin{array}{llll}\text { H } & -2.82403821210324 & 1.26579328891717 & 2.42915173542035\end{array}$

$\begin{array}{llll}\text { H } & -3.92076987179647 & -0.12489435067406 & 2.40203102428254\end{array}$

C $-2.03130981829354 \quad-1.82001245147529 \quad 2.95361408430402$

C $-0.77973275405435 \quad-2.47753483472519 \quad 3.32242822595287$

C $-0.55433512402522 \quad-3.78497848015112 \quad 3.77991978396918$

H $-1.39244185002614 \quad-4.47296266050731 \quad 3.92998743724209$

C $0.76349613099108 \quad-4.19718003162831 \quad 4.04166719845272$

C $1.83563605972649 \quad-3.30942501459590 \quad 3.84744385907049$

H $2.86608333771816 \quad-3.62180223009115 \quad 4.04658706643546$

C $1.56430310017108 \quad-2.01198237480666 \quad 3.38994899349854$

C $2.49511423979681 \quad-0.92618779092637 \quad 3.08583479184618$

$\begin{array}{llll}\text { C } & 2.58832947054534 & 1.33199560483494 & 2.16246220738551\end{array}$

H $3.60926631815658 \quad 1.39509752409972 \quad 2.56804523480549$

H $2.03474748825949 \quad 2.22288424425048 \quad 2.49136870158217$

$\begin{array}{llll}\text { C } 2.62831179710268 & 1.28984619393060 & 0.59610053009574\end{array}$

H $2.10925800430220 \quad 2.17220146992316 \quad 0.19505298725748$

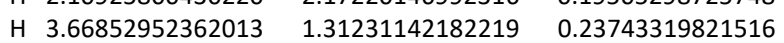

C $4.04224084290774 \quad-1.17056347179954 \quad-0.32425238024459$

H $4.59022698575951 \quad-0.55295854004766 \quad 0.40511209146636$

$\begin{array}{llll}\text { H } 4.39454469787236 & -0.88797286162590 & -1.33875594382002\end{array}$

H $4.32151189806987 \quad-2.22614022281827 \quad-0.16117016638720$

C $-3.27038478525772 \quad-2.57497842462282 \quad-0.54762849073008$

H $-4.15434138822929-1.99502466066765 \quad-0.24230825102226$ 


\begin{tabular}{|c|c|c|c|}
\hline & & & \\
\hline & & & \\
\hline & & & \\
\hline & & & \\
\hline & & & \\
\hline & & & \\
\hline & & & \\
\hline & & & \\
\hline & & & \\
\hline & & & \\
\hline & & & \\
\hline & -1.35 & & \\
\hline & $-2.4 C$ & & \\
\hline & -1.33 & & \\
\hline & 1.42 & & -2.9 \\
\hline & 1.27 & & \\
\hline & 1.6 & & \\
\hline & 2.2 & & \\
\hline & 64 & & \\
\hline & 0.45 & & \\
\hline & -1.3 & & \\
\hline & -0.3 & & \\
\hline & -0.51 & & \\
\hline & -1.46 & & 4.0 \\
\hline & 0.317 & 3.2 & 4.01 \\
\hline & -0.55 & & 5.5 \\
\hline & -1.65 & & 5.55 \\
\hline & -1.64 & 990 & 6.56 \\
\hline & 9984820584 & 9178 & 3557 \\
\hline & -2.60 & & 5.0 \\
\hline & 1.19 & 0.75 & 5.66 \\
\hline & 0.98 & 92 & 6.66 \\
\hline & $2.07 C$ & 58 & 5.23 \\
\hline & 1.41 & -0.3 & 5.740 \\
\hline & & & \\
\hline & & -5.28681781611033 & \\
\hline
\end{tabular}

$\left[\left({ }^{3} \mathrm{PDI}_{2}\right) \mathrm{Co}_{2}(\mu-\mathrm{N})\left(\mathrm{PMe}_{3}\right)_{2}\right]^{3+}\left({ }^{3}\left[\mathrm{Co}_{2} \mathrm{~N}\right]^{3+}, \mathrm{S}=0\right)$

$\mathrm{E}=-5002.23765813 E_{\mathrm{h}}$

$\begin{array}{llll}\text { Co } 0.03934522411860 & -0.09855164169774 & 0.01462299384272\end{array}$

Co $0.01035249398875 \quad-0.06252071714126 \quad 2.45349021333515$

$\begin{array}{llll}\text { P } & 0.09829125147560 & -1.25349379622316 & -1.95419425798580\end{array}$

P $0.02322566027210 \quad-1.16068246004599 \quad 4.45676063871958$

$\begin{array}{llll}\text { N } & -1.90703481651256 & 0.14369889030345 & -0.20962194930357\end{array}$

$\begin{array}{llll}\mathrm{N} & -0.02592146528458 & 1.72654130683309 & -0.54004116982558\end{array}$

$\begin{array}{llll}\text { N } & 1.94582224468433 & 0.26982840796760 & -0.15024937074915\end{array}$

$\begin{array}{llll}\text { N } & 1.91543508068021 & 0.31874853896453 & 2.65477134776153\end{array}$

\begin{tabular}{lllll} 
N & -0.07246036564852 & 1.77865474356221 & 2.95590865658223 \\
\hline
\end{tabular}

$\begin{array}{llll}\mathrm{N} & -1.94289691494891 & 0.18223849952707 & 2.62549282143397\end{array}$

$\begin{array}{llll}\text { N } & -0.06582786166050 & -1.28891919861062 & 1.25228567803497\end{array}$

C $-2.31351748241544 \quad 1.34625077607975 \quad-0.54250225427952$

$\begin{array}{llll}\text { C } & -1.23021939416674 & 2.32787540450069 & -0.64247777818035\end{array}$

C $-1.30738077255396 \quad 3.71863735868756 \quad-0.81419851930724$

H $-2.27598840113630 \quad 4.22302718178333 \quad-0.89478572063936$

$\begin{array}{llll}\text { C } & -0.10936565578134 & 4.45345907627748 & -0.87097845585976\end{array}$

$\begin{array}{llll}\text { C } & 1.13151146870424 & 3.79729884314354 & -0.78557010849977\end{array}$

H $2.06723412382868 \quad 4.36316911137103 \quad-0.84378792632885$

$\begin{array}{llll}\text { C } & 1.14139581946240 & 2.40400123919561 & -0.61378751425608\end{array}$

$\begin{array}{llll}\text { C } 2.28276948871185 & 1.49627815351605 & -0.48279587423423\end{array}$

$\begin{array}{llll}\text { C } 2.95607209710894 & -0.79181836256929 & 0.00301020242151\end{array}$

H $3.64351881393064 \quad-0.78141814719483 \quad-0.86180095747902$

$\begin{array}{llll}\text { H } & 2.42142369577674 & -1.75370026745900 & -0.00084441699866\end{array}$

$\begin{array}{llll}\text { C } 3.77105294648707 & -0.66449094387351 & 1.28689106572013\end{array}$

H $4.48958261341720-1.50594104334535 \quad 1.30967299552534$

H $4.36906921528332 \quad 0.26338493243470 \quad 1.27368957359672$ 
C 2.93263399086203

H 2.40097381368162

H 3.60368773211446

C 2.23801214253627

C 1.09037286591616

C 1.07336469102211

H 2.00662956904369

C -0.17061606710928

C -1.36519015961992

H -2.33700994793329

C -1.27974191170058

C -2.35815159990684

C -2.87826439593481

H -3.57023878197205

H -2.27914212259015

C -3.69082428540226

H -4.35905187129958

H -4.34618197777114

C -2.84739179615866

H -2.24981107177229

H -3.51927989474956

C -3.72808668194437

H -3.75797839237424

H -4.14122801638750

H -4.39416063445703

C 3.67456268361039

H 3.67555109394555

H 4.38786691977989

H 4.05230195542897

C 3.61460347671144

H 3.96301898144069

H 4.36043624524060

H 3.59003352989634

C -3.77499564325314

H -4.15250925279713

H -3.81617137534857

H -4.46136919642479

C -1.31897115914253

H -1.43814811939566

H -2.24681311458744

H -1.11752280638277

C 0.16187623185114

H -0.72134959857904

H 1.07423798095460

H 0.17150546798249

C 1.50845560665776

H 1.36450988272937

H 2.45807476863830

H 1.54658717698744

C 0.09424533318786

H 1.01838894179373

H -0.77606228288294

H 0.08462504688760

C 1.40666171414622

H 1.23927241983717

H 1.44134807382976

H 2.36613210748531

C -1.42162330815449

H -1.24536129494568

H -2.33834005014955

H -1.54550699508816

H -0.20941152184786

H -0.14296422395750
$-0.74366977958537$ $-1.70675026576008$ $-0.69964237321801$ 1.55517485802856 2.46162760928301 3.85915474306755 4.42848993521131 4.51385092095828 3.77381731020200 4.27673483640422 2.37914032347593 1.39123649538968 $-0.94734863704987$ $-0.96058184238792$ $-1.87005595272608$ $-0.91557376477590$ $-0.03665960535363$ $-1.80769001817781$ $-0.98026167715777$ $-1.90443064219410$ $-1.01439972281440$

1.75137788532500 2.41510378070814 2.33017012295113 0.89360560857321 1.98742107552885 2.68094161159725 1.17372562149112 2.55689478377063 2.06621766789577 2.73294269694880 1.26764142760089

2.67868210653471 1.80307142578927 2.43526974746511 2.42335926888433 0.95130769551654 $-0.93498284604019$ 0.15471335098357 $-1.35840936681802$ $-1.40832353851374$ $-3.05834356965502$

$-3.39208078778987$ $-3.33193371084247$

$-3.55296154879904$ $-0.83047236059577$ $-1.31930263732280$ $-1.17976621261768$ 0.26503676949589 $-2.96987537679930$ $-3.25428805739083$

$-3.31914346676282$ $-3.44122325091171$ $-0.70848728139040$ $-1.17273230563415$ 0.39037541322523 $-1.06848227813167$ $-0.81727743134474$ $-1.26589053338312$ $-1.25207284135614$ 0.27530582221962 5.60567610960508 5.54234077318280
2.56002888961298 2.59000971162689 3.43642371790680 2.96270331032161 3.03817952886937 3.16906146687314 3.23330612075779 3.20655702520144 3.14324999544965 3.18673202577795 3.01357845514498 2.91955976342368 2.49489256002907 3.35570268565032 2.52572947851664 1.20137185714994 1.18219981398551 1.20483442464012 $-0.07086080228861$ $-0.06406935107669$ $-0.94715067236530$ $-0.82614925772477$ $-1.71050270024146$ 0.02458220112658 $-1.00897332441575$ $-0.73979729075984$ $-1.60099914745732$ $-0.94669404507758$ 0.13351437070840 3.26027473208234 2.44545349199418 3.39261874318120

4.18209859239331 3.18121881719637 2.35206033409075 4.09715755805063 3.30382977068192 $-3.05608544855354$ $-3.19752053727024$

$-2.64053814478123$

$-4.03596133981690$

$-1.74116703015894$

$-1.16752231465109$

$-1.18108497335323$

$-2.73189194837619$ $-3.03032106523419$ $-4.01279490654343$ $-2.59552669172742$ $-3.17288495992977$ 4.28962477654530 3.75495432582957

3.70566688879759 5.29167783178259 5.55502244857088 6.54565017295545 5.67084407021521 5.15166023538404 5.51451471921735 6.51081476507008 5.08619089649393 5.62659609570908 3.29362945392706 $-0.99075008418510$ 
$\left[\left({ }^{2} \mathrm{PDI}_{2}\right) \mathrm{Co}_{2}(\mu-\mathrm{N})\left(\mathrm{PMe}_{3}\right)_{2}\right]^{3+}\left({ }^{2}\left[\mathrm{Co}_{2} \mathrm{~N}\right]^{3+}, \mathrm{S}=0\right)$ $\mathrm{E}=-4923.77375715 E_{\mathrm{h}}$

Co $0.11838804186428 \quad-0.36750831919906$

$\begin{array}{llll}\text { Co } 0.02037773734481 & -0.35395976820388 & 2.54413147136428\end{array}$

$\begin{array}{llll}\text { N } & -0.15238486422781 & 0.79046207473978 & 1.28576247480988\end{array}$

$\begin{array}{llll}\text { P } & 0.09416438417072 & 1.17114031920687 & -1.59720778079221\end{array}$

$\begin{array}{llll}\text { P } & -0.50674192606069 & 1.10629730615862 & 4.18879353657266\end{array}$

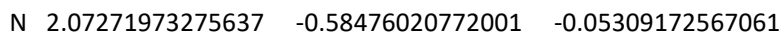

N $0.22405162926029 \quad-1.90423605130728 \quad-1.06955191266963$

N $-1.75307753582944 \quad-0.80330030613753 \quad-0.10573651277862$

$\begin{array}{llll}\mathrm{N} & -1.72082095553242 & -1.26916305830534 & 2.65865088868777\end{array}$

$\begin{array}{llll}\text { N } & 0.47233914149811 & -1.79605779804591 & 3.70830089136772\end{array}$

N $1.92123703810890 \quad-0.07863455214645 \quad 2.70683827931195$

C $2.50468953658824 \quad-1.54046893629752 \quad-0.85531286038883$

C $1.44162947411244 \quad-2.33369599778886 \quad-1.46978840634104$

C $1.53673120338954-3.41189374319593 \quad-2.36711924406280$

H $2.50973974208279 \quad-3.78014896454338$ - 2.70736617151154

C $0.35324506896211 \quad-4.01454355465021 \quad-2.82527167888657$

C $-0.89890328711994 \quad-3.54367126552861 \quad-2.39163503659863$

$\begin{array}{llll}\text { H } & -1.82529445632228 & -4.00905871800448 & -2.74408918582316\end{array}$

C $-0.93119822947777 \quad-2.46488086762370 \quad-1.49617883283545$

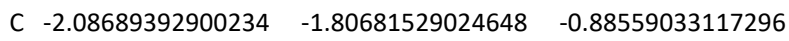

$\begin{array}{llll}\text { C } & -2.72385197848145 & -0.07087539227753 & 0.70804905575803\end{array}$

$\begin{array}{llll}\text { H } & -3.68844100569220 & 0.06256349481112 & 0.18903358051861\end{array}$

$\begin{array}{llll}\text { H } & -2.30448309368262 & 0.92926720350801 & 0.90148115889828\end{array}$

$\begin{array}{llll}\text { C } & -2.96348524773714 & -0.81949454021341 & 2.01538067254843\end{array}$

H $-3.51995803223953 \quad-0.16050283085688 \quad 2.71178260580591$

H $-3.60653895395191 \quad-1.69295754993320 \quad 1.81254419163784$

C $-1.78121950109107 \quad-2.29751317169930 \quad 3.48428501853832$

C $-0.50642285180823 \quad-2.63511125049811 \quad 4.11522253529323$

C $-0.20651992867328 \quad-3.65831230501506 \quad 5.03160280726375$

H $-0.98015399336632 \quad-4.35179848568524 \quad 5.37606884716400$

$\begin{array}{llll}\text { C } & 1.11268253079170 & -3.77929230993243 & 5.49951770752520\end{array}$

$\begin{array}{llll}\text { C } & 2.10939456771286 & -2.89245991181538 & 5.05498678859151\end{array}$

H $3.14011243158763 \quad-2.98444731730525 \quad 5.41287534045448$

C $1.75119470059787 \quad-1.89159390032062 \quad 4.14011914769960$

C $2.59251258085643 \quad-0.87395308335435 \quad 3.50908968728855$

C $2.56467061825060 \quad 0.93606030724551 \quad 1.87080691496466$

H $3.41465591167079 \quad 1.42169736377304 \quad 2.38059803247371$

H $1.81461206285479 \quad 1.71459069235091 \quad 1.65823644113275$

$\begin{array}{llll}\text { C } 3.06875417176401 & 0.28837550687728 & 0.58487679870255\end{array}$

$\begin{array}{llll}\text { H } 3.37743872576294 & 1.07909342688237 & -0.12763790323348\end{array}$

H $3.97182244618309 \quad-0.30169526982704 \quad 0.81720009186789$

C $3.95291650724288 \quad-1.79743896153808 \quad-1.14025560205210$

H $4.45006957119104 \quad-0.88676918141184 \quad-1.52766021680594$

H $4.07866816740838 \quad-2.60203924429446 \quad-1.88228238262241$

H $4.49039472179150 \quad-2.10120903522221 \quad-0.21856184282182$

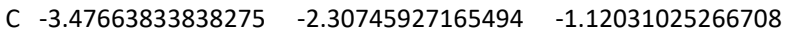

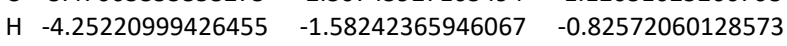

H $-3.63881941573918 \quad-3.24269793178608 \quad-0.54430003696623$

H $-3.61613422721496 \quad-2.55649067562235 \quad-2.18891078184158$

$\begin{array}{llll}\text { C } & -3.04059294170700 & -3.05146084207356 & 3.78433082708810\end{array}$

H $-2.86585752491232 \quad-3.84823620177532 \quad 4.52499589623842$

$\begin{array}{llll}H & -3.44718450571469 & -3.52611408213978 & 2.86812761008682\end{array}$

H $-3.82289476529026 \quad-2.37476994414930 \quad 4.18122029116019$

$\begin{array}{llll}\text { C } 4.06837815028605 & -0.83406014089415 & 3.74950735727137\end{array}$

$\begin{array}{llll}\text { H } & 4.53332589389216 & 0.11235767923481 & 3.43077513776578\end{array}$

H $4.55677310303924 \quad-1.66434196513844 \quad 3.19756784178853$

H $4.28445375759382 \quad-0.98689978971544 \quad 4.82357529728437$

$\begin{array}{llll}\text { C } & 0.73538075801355 & 2.78693948355471 & -1.07006772859854\end{array}$

H $0.63799093312288 \quad 3.51296890410496 \quad-1.90002807622375$

$\begin{array}{llll}\text { H } & 1.80010892118426 & 2.69744947350553 & -0.79108371003907\end{array}$

H $0.15833623468610 \quad 3.13431915275796 \quad-0.19450732394619$

$\begin{array}{llll}\text { C } & -1.57232832901779 & 1.50500061177472 & -2.24198190364693\end{array}$

H $-1.51171806032313 \quad 2.25828671926827 \quad-3.05103367124189$ 


$\begin{array}{llll}\text { H } & -2.21178352923086 & 1.89314476358663 & -1.42936667628508 \\ \text { H } & -2.01142671895786 & 0.57336672821864 & -2.64158020485991 \\ \text { C } & 1.07411568195625 & 0.69724233599162 & -3.05401261302945 \\ \text { H } & 0.66205657912128 & -0.22879464487658 & -3.49467114465325 \\ \text { H } & 2.12707625620227 & 0.52845593541081 & -2.76541214166292 \\ \text { H } & 1.03059449651774 & 1.50869167248694 & -3.80585283272951 \\ \text { C } & -1.66920742079211 & 0.44069709713581 & 5.41963871676364 \\ \text { H } & -1.25651016833388 & -0.48273863297657 & 5.86507651078160 \\ \text { H } & -2.64119372050445 & 0.21730049845296 & 4.94546420307938 \\ \text { H } & -1.82057010653345 & 1.19044876651105 & 6.21984542647560 \\ \text { C } & 0.93273952778589 & 1.64262896631833 & 5.16177564879340 \\ \text { H } & 0.59863490737804 & 2.32014489207269 & 5.97104578119648 \\ \text { H } & 1.64666386217306 & 2.17499961675641 & 4.50841311135095 \\ \text { H } & 1.43017825795186 & 0.76258502107880 & 5.60865190928829 \\ \text { C } & -1.26802859800533 & 2.63080554349613 & 3.56249536324367 \\ \text { H } & -1.50956216521896 & 3.30201511883982 & 4.40908611591540 \\ \text { H } & -2.19231572701094 & 2.38324523201091 & 3.01041756001240 \\ \text { H } & -0.56341659956730 & 3.12972673693222 & 2.87368953216685 \\ \text { H } & 1.36644587703369 & -4.57125525033300 & 6.21344522437870 \\ \text { H } & 0.40679698327499 & -4.85643447231303 & -3.52498876413582\end{array}$

$\left[\left({ }^{3} \mathrm{PDI}_{2}\right) \mathrm{Co}_{2}\left(\mu-\mathrm{NH}_{2}\right)\left(\mathrm{PMe}_{3}\right)_{2}\right]^{3+}\left({ }^{3}\left[\mathrm{Co}_{2} \mathrm{NH}_{2}\right]^{3+}, \mathrm{S}=0\right)$

$\mathrm{E}=-5003.49709632 E_{\mathrm{h}}$

Co $-0.02054246288410 \quad-0.02586599474407 \quad 0.01417214127905$

Co $-0.00947918330067 \quad 0.00155570317616 \quad 2.52092377291903$

$\begin{array}{llll}\text { P } & 0.02488312590106 & -1.04248844056617 & -1.98730126268606\end{array}$

P $0.05320959597973 \quad-0.96395896457459 \quad 4.54724433635025$

N $-1.95041543456392 \quad 0.14236280954596 \quad-0.11949956346286$

N $\quad-0.10335430624560 \quad 1.74877404208615 \quad-0.41264383492518$

N $1.88232275401523 \quad 0.31853009278310 \quad-0.13475461182164$

$\begin{array}{llll}\text { N } 1.89684577269649 & 0.35567275242963 & 2.64957923830322\end{array}$

$\begin{array}{lllll}N & -0.09461727846867 & 1.78345918167267 & 2.91867623475639\end{array}$

$\begin{array}{llll}\mathrm{N} & -1.93880572032881 & 0.16733696174108 & 2.66523229938658\end{array}$

N $0.04952925788151 \quad-1.46912221639753 \quad 1.28395174274620$

C $-2.39017633969061 \quad 1.34502740484667 \quad-0.42624274929480$

C $-1.32415556722981 \quad 2.33457041646771 \quad-0.53201698601637$

C $-1.41591774874327 \quad 3.71849203396177 \quad-0.74173493476164$

H $-2.39421967050195 \quad 4.20318369424256 \quad-0.82571484649661$

C $-0.23141124972272 \quad 4.46973555485156 \quad-0.83418389002942$

C $1.01820723044992 \quad 3.83146980319525 \quad-0.75206711070484$

H $1.94602286998750 \quad 4.40565015421652 \quad-0.84463288294318$

C $1.05744025551237 \quad 2.44468003756247 \quad-0.54163819140353$

C $2.20999100261640 \quad 1.55690875845840 \quad-0.44209177863742$

C $2.91145369105313 \quad-0.72526408369315 \quad-0.01911412057595$

H $3.58850305114735 \quad-0.68659236797911 \quad-0.89188444874392$

H $2.40288148655858-1.70124300773542 \quad-0.05545137622156$

C $3.74445419763878 \quad-0.61898777561055 \quad 1.25848333039768$

H $4.45800333498814 \quad-1.46485298062508 \quad 1.26615210282684$

$\begin{array}{llll}\text { H } & 4.34794808263068 & 0.30529561738574 & 1.23872431906587\end{array}$

C $2.92861536596461 \quad-0.68806473801493 \quad 2.55029095431864$

H $2.42215295883840 \quad-1.66337774164717 \quad 2.61942507526041$

H $3.61642037814003 \quad-0.62360054765997 \quad 3.41275533967236$

C $2.22009110191513 \quad 1.59940809577349 \quad 2.93704782631234$

C $1.06450478316930 \quad 2.48430690639096 \quad 3.02960158840367$

C $1.02412873010154 \quad 3.87340071343162 \quad 3.22217730228579$

H $1.95194138580719 \quad 4.44953055389167 \quad 3.30149227933344$

C $-0.22645020152756 \quad 4.50988492338602 \quad 3.30364414048074$

C $-1.40956670710596 \quad 3.75462545703577 \quad 3.22834923741966$

H $-2.38821615285823 \quad 4.23871228361548 \quad 3.31182846421721$

C $-1.31615019289452 \quad 2.36793051235018 \quad 3.03730326361542$

C $-2.37997482750315 \quad 1.37409119425043 \quad 2.95351334048376$

$\begin{array}{llll}\text { C } & -2.86578112020261 & -0.97116276434922 & 2.57118033005157\end{array}$

H $-3.54580470819994 \quad-0.97475673143186 \quad 3.44187732087002$

H $-2.26761739282692 \quad-1.89398267544609 \quad 2.63028865890607$

C $-3.70107977389499 \quad-0.97460679330602 \quad 1.28989201950661$ 


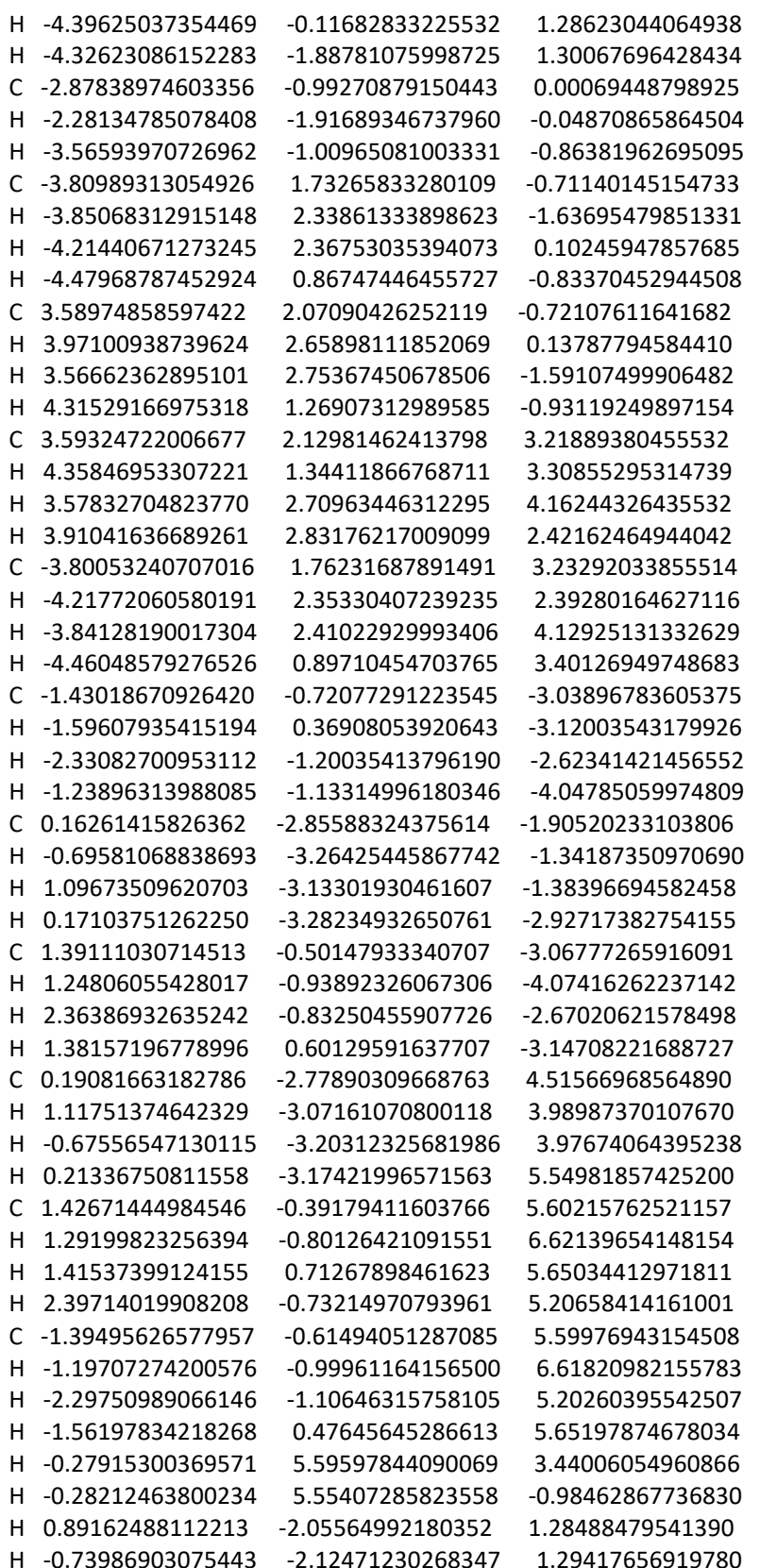

$\left[\left({ }^{2} \mathrm{PDI}_{2}\right) \mathrm{CO}_{2}\left(\mu-\mathrm{NH}_{2}\right)\left(\mathrm{PMe}_{3}\right)_{2}\right]^{3+}\left({ }^{2}\left[\mathrm{CO}_{2} \mathrm{NH}_{2}\right]^{3+}, \mathrm{S}=0\right)$ $\mathrm{E}=-4925.02801039 E_{\mathrm{h}}$
Co 0.02999851736603
Co 0.04702016107229
N -0.20873894216432
$-0.08950871380493$
$-0.10663154785128$
1.33194120808597
0.05520226045494
2.54412872250993
1.30203814802611
P -0.16356575557599
1.02483227813063
$-1.86928392528819$
P -0.13715228018500
1.03391176866583
4.44605520255909
N 1.95319004640563
$-0.03115869195093$
$-0.01427996862438$
N 0.31920536762015
$-1.76447334445111$
$-0.61102506481649$
N -1.79875753537051
$-0.68594133321996$
0.00737744646812
N -1.77870015613803
$-0.70290958922451$
2.61525980331508
N 0.33495381641183
$-1.76579173530062$
3.25621368067795
N 1.96839836910021
$-0.06275712538659$
2.59400446480691
C $2.54351673631165-1.09881199609424$
$-0.51691602985599$
C 1.59770858655867
$-2.14577565916467$
$-0.88695404595291$ 
C $1.83842702941108 \quad-3.40905085458649$

H $2.85876293034495-3.735035920844005$

C $0.74741109698590-4.25104382000526$

C $-0.56176138182661 \quad-3.82720162127401$

H $-1.41374007964899-4.47917472928302$

C -0.75745901296258

C -1.99814929132122

C -2.90763492361337

H -3.87511631791134

H -2.80773841784475

C -2.89019367335687

H -2.76833836880360

H -3.85413624289518

C -1.98038313490184

C -0.74120675394279

C -0.54547786931715

H -1.39795531248460

C 0.76387307588365

C 1.85530561819833

H 2.87680136273177

C 1.61443399761635

C 2.56003691753448

C 2.74747692410658

H 3.78484997439400

H 2.29912229824086

C 2.73782860695512

H 2.28723299192789

H 3.77034561791046

C 4.02406641106985

H 4.41030353413620

H 4.27433486409408

H 4.57299198682013

C -3.34205938915623

H -3.89935516738718

H -3.24401665432829

H -3.96511769275560

C -3.32588345095015

H -3.90998355473445

H -3.22860115316268

H -3.92204807630386

C 4.03838422068264

H 4.39705769615105

H 4.60819524072348

H 4.29290743824431

C -1.79596358787831

H -1.76035708648937

H -2.05034026755829

H -2.56749069642007

C 0.14951994765102

H 0.07162576487432

H -0.59488162008915

H 1.16318332056652

C 0.99071864652895

H 2.03013592292731

H 0.87113130820396

H 0.76841293902078

C -1.48681990814196

H -1.35189545016442

H -2.46194958734068

H -1.46564081560327

C 1.34237309015222

H 1.14477991171458

H 2.20028691227383

H 1.58246240116156

C -0.43408346442204

H -0.49606714580778 \begin{tabular}{ll}
-1.65632734702127 \\
\hline
\end{tabular}

$-2.55611607308642 \quad-0.87575560636068$

$-1.89021640580884 \quad-0.49330017938729$

$0.09838963412853 \quad 0.54606538562379$

$-0.27910116363504 \quad 0.18364365756476$

$1.13163318056309 \quad 0.18532161240078$

$0.09420483785962 \quad 2.11003195303336$

$1.12405692319043 \quad 2.48253959757120$

$-0.27327106155598 \quad 2.49179911284507$

$-1.89395360058799 \quad 3.14340078588983$

$-2.55297082103696 \quad 3.53733762265831$

$-3.81430774789319 \quad 4.12147326654897$

$-4.46136280725408 \quad 4.35148795054714$

$-4.23683158368372 \quad 4.40470211943512$

$-3.40396509782635 \quad 4.10769938876184$

$-3.73080844203662 \quad 4.32678610282331$

$-2.15036036872598 \quad 3.52402090995838$

$-1.11765639549718 \quad 3.11856941575897$

$1.05910219446885 \quad 2.08319400540643$

$1.02544721045063 \quad 2.44745468030998$

$1.98782114185423 \quad 2.47100213411960$

$1.07975407540160 \quad 0.51975452892848$

$2.01853825340338 \quad 0.17095463181968$

$\begin{array}{ll}1.05614634828091 & 0.14099872712580\end{array}$

$-1.24834091827082-0.70539749604119$

$-0.48055381779348-1.40456538871059$

$-2.23871993820201-1.11880906377518$

$\begin{array}{ll}-1.13366939566499 & 0.24840146888175\end{array}$

$-2.53066773188724 \quad-0.67621304146783$

$\begin{array}{ll}-2.60462999890384 & 0.27677148697232\end{array}$

$-3.54960896287864-1.08424692388557$

$-1.94316083032171-1.37961514773895$

$\begin{array}{ll}-2.51665607437053 & 3.36727714547809\end{array}$

$-1.92950559115954 \quad 4.10447297449271$

$-3.54293055837050 \quad 3.75686697371682$

$\begin{array}{ll}-2.56736134598719 & 2.43712849626793\end{array}$

$-1.25675266812917 \quad 3.32924944262313$

$-0.51690705890968 \quad 4.07312079704672$

$-1.09121480456609 \quad 2.39614470588725$

$-2.26118380451589 \quad 3.70454452352265$

$1.74915055944572 \quad-2.22308508755566$

$2.23778563580060 \quad-3.21602349909468$

$2.50509934807728 \quad-1.45877863710780$

$0.95910008328156 \quad-2.23854965365450$

$-0.07954639455436 \quad-3.28126246350369$

$0.49100467633284 \quad-4.22645917223051$

$-0.89647460538607 \quad-3.28611327513231$

$-0.51179224133201-3.19967729511131$

$2.41090284426015 \quad-2.11262195325136$

$2.03858813326642 \quad-2.12921226071581$

$3.15282455336070-1.30254829806237$

$2.89643526678675 \quad-3.08262828785002$

$0.44475685357339 \quad 5.51623250960828$

$\begin{array}{ll}-0.63293000460577 & 5.72154855636847\end{array}$

$0.60312807411325 \quad 5.02317480060527$

$1.00081630884944 \quad 6.47295396704889$

$0.91882029833236 \quad 5.50004248298049$

$1.42216210149233 \quad 6.46577200425690$

$1.40559414959598 \quad 5.00371952812759$

$-0.14389137564446 \quad 5.68650643821411$

$2.81711782093443 \quad 4.23872504352490$

$3.30174897626875 \quad 5.23230061015984$ 


$\begin{array}{cccc}\text { H } & -1.38196712267845 & 2.97795641048317 & 3.69372210907358 \\ \text { H } & 0.39503898131096 & 3.26746611639301 & 3.66336913023423 \\ \text { H } & 0.93441536040499 & -5.21939772182948 & 4.85905460259424 \\ \text { H } & 0.91764435093088 & -5.24056702466616 & -2.16087154029379 \\ \text { H } & -1.11638013031943 & 1.80882913931142 & 1.31287190553260 \\ \text { H } & 0.48943717922491 & 2.08290471457239 & 1.29917775599568\end{array}$

$\left[\left({ }^{2} \mathrm{PDI}_{2}\right) \mathrm{Co}_{2}\left(\mu-\mathrm{NPMe}_{3}\right)\left(\mathrm{PMe}_{3}\right)_{2}\right]^{3+}\left({ }^{2}\left[\mathrm{Co}_{2} \mathrm{NP}\right]^{3+}, \mathrm{S}=0\right)$

$\mathrm{E}=-5384.69992949 E_{\mathrm{h}}$

Co $0.02373351051113 \quad-0.01581632926919 \quad-0.00875754393407$

Co $-0.01005947428045 \quad 0.07017475488272 \quad 2.58293104963090$

$\begin{array}{lllll}\text { P } & -0.19783260448798 & 1.09982363850049 & -1.93225138193071\end{array}$

$\begin{array}{llll}\text { P } & 0.34346744035496 & -2.13534931538868 & 2.86829362393077\end{array}$

$\begin{array}{lllll}P & -0.59057068702357 & 2.92336538018717 & 1.61031917325933\end{array}$

$\begin{array}{llll}\text { N } & 1.92569212178205 & 0.04042265115028 & -0.07950336738160\end{array}$

N $0.32752632215751 \quad-1.63376067949799 \quad-0.82087182009870$

N $-1.76873181333435 \quad-0.63688147793656 \quad-0.10633585519550$

$\begin{array}{llll}N & -1.98752532840390 & -0.08918750079265 & 2.88808836704990\end{array}$

$\begin{array}{llll}N & -0.07783343586140 & 0.42771251836656 & 4.40853639456628\end{array}$

$\begin{array}{llll}\text { N } & 1.92588284399732 & 0.52259699516019 & 2.92528065317981\end{array}$

$\begin{array}{llll}\text { N } & -0.17435927061747 & 1.43642432090984 & 1.22846042735111\end{array}$

C $2.53476760271743 \quad-0.94111697932094 \quad-0.71723327670375$

C $1.60248104824151-1.95226059754638$ - 1.18943241744730

C $1.84567908291445-3.12412374188064 \quad-1.92152691338651$

H $2.86462573404184 \quad-3.39410078206794 \quad-2.21547060129864$

C $0.76128238062395 \quad-3.94362026603688 \quad-2.27581418692148$

C $-0.54592223959185 \quad-3.57587809186181 \quad-1.91939494212392$

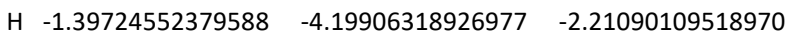

$\begin{array}{llll}\text { C } & -0.74432240479065 & -2.39301659076413 & -1.19112336002781\end{array}$

C $-1.98018681585901 \quad-1.77912887208130 \quad-0.73482307815817$

$\begin{array}{llll}\text { C } & -2.86299817846096 & 0.08409355063563 & 0.51114906031766\end{array}$

H $-3.80917434387767 \quad-0.07134118194110 \quad-0.03504827683663$

$\begin{array}{llll}\text { H } & -2.62353424762601 & 1.15078855558394 & 0.49175612474848\end{array}$

$\begin{array}{llll}\text { C } & -3.08044156132784 & -0.37473318331129 & 1.94353081334972\end{array}$

$\begin{array}{llll}\text { H } & -4.00336295048410 & 0.11895681504203 & 2.28808865688019\end{array}$

$\begin{array}{llll}H & -3.27364033564402 & -1.46430621452671 & 1.95926101912848\end{array}$

$\begin{array}{llll}\text { C } & -2.35311115606512 & 0.02131391173115 & 4.15209704772807\end{array}$

C $-1.26605882586637 \quad 0.32031882473351 \quad 5.06364205917740$

C $-1.32988293951169 \quad 0.48552844003829 \quad 6.45719244351704$

$\begin{array}{llll}\text { H } & -2.28664285333293 & 0.40770942443959 & 6.98243481906003\end{array}$

$\begin{array}{llll}\text { C } & -0.15278368744446 & 0.74967662897151 & 7.16967915672629\end{array}$

C $1.06674777441195 \quad 0.82412892006539 \quad 6.48214086452554$

$\begin{array}{llll}\text { H } & 1.99639993205366 & 1.01418747354197 & 7.02600794284284\end{array}$

C $1.07928838777948 \quad 0.65431412060931 \quad 5.08915351856429$

C $2.22241801481146 \quad 0.70475511059154 \quad 4.19661530760716$

C $3.05404776498212 \quad 0.66330003715719 \quad 1.99038527176425$

H $3.60311659629386-0.29719750118445 \quad 1.96071070458687$

H $3.75319927114959 \quad 1.42715730029467 \quad 2.36996803601342$

$\begin{array}{llll}\text { C } 2.68600696779371 & 1.08593345800790 & 0.57623882512595\end{array}$

H $2.07013270328092 \quad 1.99355707145371 \quad 0.61382873771107$

H $3.62190421464115 \quad 1.30683234954424 \quad 0.03405749397393$

$\begin{array}{llll}\text { C } 4.01596974501780 & -1.02537636924070 & -0.93345413252762\end{array}$

H $4.28652403256249-1.93635784635577 \quad-1.49247718116991$

H $4.37912911498943 \quad-0.15101217832387 \quad-1.51042403110681$

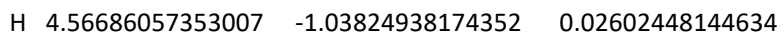

C $-3.32998416041248 \quad-2.39041453079730 \quad-0.95840771693273$

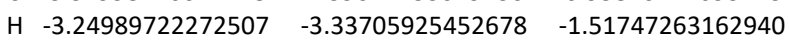

$\begin{array}{llll}\text { H } & -3.83991521446315 & -2.60599570763814 & 0.00032183326894\end{array}$

$\begin{array}{llll}\text { H } & -3.98330055005016 & -1.70657845541732 & -1.53620657394418\end{array}$

$\begin{array}{llll}\text { C } & -3.75990947800076 & -0.15899574128935 & 4.64407151858345\end{array}$

H $-3.80520667201758 \quad-0.16014765170881 \quad 5.74511512414034$

H $-4.41892851040243 \quad 0.65828344616729 \quad 4.28325630421656$

H $-4.18718013686581 \quad-1.11383381386469 \quad 4.28192855410341$

$\begin{array}{llll}\text { C } 3.60707450703322 & 0.96437114101132 & 4.71532237824815\end{array}$

H $3.64653304911926 \quad 0.88491619717307 \quad 5.81414788886451$ 


\begin{tabular}{|c|c|c|c|}
\hline & & & \\
\hline & & & \\
\hline & & & \\
\hline & & & \\
\hline & & & \\
\hline & 530 & & \\
\hline & & & \\
\hline & & & \\
\hline & & & \\
\hline & & & \\
\hline & & & \\
\hline & & & \\
\hline & & & \\
\hline & & & \\
\hline & & & \\
\hline & & & \\
\hline & & & \\
\hline & & & \\
\hline & & & \\
\hline & & & \\
\hline & 33 & & \\
\hline & & & \\
\hline & & -2.7 & \\
\hline & & -2.6 & \\
\hline & & -2.2 & \\
\hline & 2.00 & -3.87 & \\
\hline & & & \\
\hline & -2.4 & & 814 \\
\hline & -2.90906 & & 151 \\
\hline & -2.7 & & \\
\hline & 0.15 & 3.45 & \\
\hline & -0.0 & 706 & 662 \\
\hline & 619 & 2.91 & 5625 \\
\hline & 1.23 & 3.24 & 2107 \\
\hline & -0.02 & 4.18 & 8237 \\
\hline & -0.3 & 5.1 & 9909 \\
\hline & 1.07 & 4.16 & 9375 \\
\hline & & & -0.5 \\
\hline & & & \\
\hline & & & \\
\hline
\end{tabular}

$\left[\left({ }^{2} \mathrm{PDI}_{2}\right) \mathrm{Co}_{2}\left(\mu-\mathrm{NPMe}_{3}\right)\left(\mathrm{PMe}_{3}\right)_{2}\right]^{3+}\left({ }^{2}\left[\mathrm{Co}_{2} \mathrm{NP}\right]^{3+}, \mathrm{S}=0\right)$

$\mathrm{E}=-5384.69910046 E_{\mathrm{h}}$

Co $0.02373402345524 \quad-0.01581545306311 \quad-0.00875697656960$

Co $-0.01005448040571 \quad 0.07016454542210 \quad 2.58292817140994$

$\begin{array}{llll}\text { P } & -0.19783548864525 & 1.09982503935203 & -1.93224868493296\end{array}$

$\begin{array}{llll}\text { P } & 0.34347277308611 & -2.13535466982422 & 2.86828907954055\end{array}$

$\begin{array}{llll}P & -0.59057546917434 & 2.92336175220117 & 1.61032676187187\end{array}$

N $1.92569240207153 \quad 0.04042428977733 \quad-0.07950570462908$

N $0.32752626097362 \quad-1.63376034148674 \quad-0.82087040008903$

N $-1.76873088315108 \quad-0.63688211688911 \quad-0.10633391932959$

$\begin{array}{llll}N & -1.98752103100865 & -0.08919522871862 & 2.88808650777384\end{array}$

$\begin{array}{llll}N & -0.07782824017476 & 0.42770864365845 & 4.40853265236384\end{array}$

$\begin{array}{llll}N & 1.92588888405240 & 0.52258556490832 & 2.92527795511149\end{array}$

$\begin{array}{llll}\mathrm{N} & -0.17435899042212 & 1.43642187476149 & 1.22846801120598\end{array}$

$\begin{array}{llll}\text { C } 2.53476743678148 & -0.94111498569773 & -0.71723766728311\end{array}$

C $1.60248093333467 \quad-1.95225928368851 \quad-1.18943433866589$

$\begin{array}{llll}\text { C } & 1.84567862445121 & -3.12412283504787 & -1.92152850250644\end{array}$

H $2.86462491276279-3.39409920215443 \quad-2.21547449770345$

C $0.76128221931417 \quad-3.94362158869992 \quad-2.27581129644609$

C $-0.54592181225160 \quad-3.57588033990152 \quad-1.91938954124177$

H $-1.39724473406615 \quad-4.19906729950766 \quad-2.21089276072690$

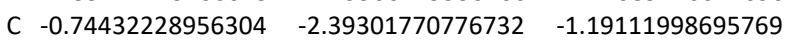

C $-1.98018642118678 \quad-1.77912945791082 \quad-0.73482122763914$

$\begin{array}{llll}\text { C } & -2.86299799174407 & 0.08409089973019 & 0.51114998262835\end{array}$ 
H -3.80917452639618

H -2.62353665597849

C -3.08043940527001

H -4.00335983055603

H -3.27363898690124

C -2.35310691548967

C -1.26605444172649

C -1.32988053013684

H -2.28664131493007

C -0.15278193303771

C 1.06675038274377

H 1.99640218081433

C 1.07929217470995

C 2.22242288497589

C 3.05405344162789

H 3.60312001345149

H 3.75320681258628

C 2.68600905051405

H 2.07013480001532

H 3.62190502621953

C 4.01596980606055

H 4.28652322450567

H 4.37913284287642

H 4.566855976802342

C -3.32998364776045

H -3.24989855426004

H -3.83990678224573

H -3.98330771825522

C -3.75990646046076

H -3.80520154758591

H -4.41891685935621

H -4.18718899200925

C 3.60707816305827

H 3.64653861989010

H 4.33610938131077

H 3.94518364553347

C -1.75992603560551

H -1.95580427472424

H -1.72057207422066

H -2.58681231470616

C 1.16312306615161

H 2.08578374888025

H 0.93339035724254

H 1.32520683440396

C -0.21653854117329

H 0.73428164955414

H -1.05658471658914

H -0.34088706791681

C -0.89451987281329

H -0.96677444165823

H -0.60023635846015

H -1.87926387091830

C 0.35466765936175

H 1.15766598689586

H -0.61801091356898

H 0.52589139144483

C 1.94466929354704

H 2.06122941919713

H 2.76342017372289

H 2.00326982070898

C -2.36025256208235

H -2.46006379028075

H -2.90906938989635

H -2.79974778026175

C 0.15085529894881

H -0.01940957444288
$-0.07134561248288$ 1.15078638546157 $-0.37473772246221$ 0.11895305426968 $-1.46431071317746$ 0.02131347770489 0.32032227006767 0.48554806860294 0.40773690026699 0.74970281005043 0.82414574122061 1.01420818289719 0.65431666899271 0.70475027950516 0.66329108123761 $-0.29720757845819$ 1.42714602932146 1.08593227043531 1.99355568384391 1.30683419160455 $-1.02537559292369$

$-1.93635333170585$ $-0.15100761322507$

$-1.03825712383208$ $-2.39041486126558$ $-3.33704985018294$ $-2.60601443609372$ $-1.70657155159560$ $-0.15898642423022$ $-0.16014740792210$ 0.65830411167496 $-1.11381656850762$ 0.96436894349910 0.88489347016035 0.24606681948781 1.98551753252406 2.01675436525731 2.70353365765357 2.59329084776136

1.28592453668803

2.23648684233924

1.64056801013303

2.74363979134143 2.99003134409065 $-0.01725954604518$ $-0.57571103198623$

$-0.73022664424354$ 0.58557097703571 $-3.30054744767106$ $-3.22816931609500$ $-4.33189487404581$ $-3.08323553490345$ $-2.58736476671241$

$-2.04678960897286$

$-2.33473301167307$

$-3.67637074475085$

$-2.79947079050417$

$-2.67468835086786$

$-2.26946801085409$

$-3.87637303139576$

3.30998238224079 4.34078088764917 3.24418500333415 2.60507505162385 3.45449217173806 4.53990174192550
$-0.03504599285020$ 0.49175700780553 1.94353082130305 2.28809005544910 1.95925980643530

4.15209398286005

5.06363813871605

6.45718664249558 6.98242892465332

7.16967222980052

6.48213454953776

7.02600099820832

5.08914888882845

4.19661139396720

1.99038171822446

1.96070206605533

2.36996574510981

0.57623856056802

0.61383488587712

0.03405600446402

$-0.93345737369738$

$-1.49248719475097$

$-1.51041930284417$

0.02602196082528

$-0.95840610811218$

$-1.51748796900032$

0.00032390372836

$-1.53618778788158$

4.64406911359749

5.74511298604221

4.28326282716119

4.28191974587821

4.71532020113112

5.81414431047565

4.29524754168377

4.44163754733657

$-2.14290600830421$

$-1.30495262905836$

$-3.08706942060097$

$-2.20236294615467$

$-2.34990475981232$

$-2.47023203479898$

$-3.30668980162858$

$-1.56588471662933$

$-3.37308960846561$

$-3.43329389838079$

$-3.29666711984465$

$-4.29283573630222$

2.21037525559412

1.11377591045521

2.48317729575431

2.65897051652416

4.64036468191190

5.17127631821671

5.09939626902249

4.73894073058365

2.29923869768578

1.21003705544802

2.81727815084731

2.54858673757944

1.84621819927987

2.23623552730253

0.88937167641214

2.57422891564337

3.17940565275683

3.30463410749165 


$\begin{array}{clcl}\text { H } & -0.31849628874694 & 2.91764009423392 & 4.02030864915745 \\ \text { H } & 1.23460992400426 & 3.24809919932933 & 3.16236691510481 \\ \text { C } & -0.02772141957429 & 4.18119253326964 & 0.42619169475557 \\ \text { H } & -0.34458482609069 & 5.17109458337381 & 0.80347176024928 \\ \text { H } & 1.07448597301644 & 4.16010439242000 & 0.35663389191801 \\ \text { H } & -0.47174188501523 & 4.03517968140926 & -0.57130247070669 \\ \text { H } & -0.18389249738876 & 0.88755818505496 & 8.25646110921681 \\ \text { H } & 0.93526214400372 & -4.86516325339867 & -2.84247901449120\end{array}$

$\left[\left({ }^{2} \mathrm{PDI}_{2}\right) \mathrm{Co}_{2}\left(\mu-\mathrm{NPMe}_{3}\right)\left(\mathrm{PMe}_{3}\right)_{2}\right]^{3+}\left({ }^{2}\left[\mathrm{Co}_{2} \mathrm{NP}\right]^{3+}, \mathrm{S}=1,\left\langle\mathrm{~S}^{2}\right\rangle=2.209417\right)$ $\mathrm{E}=-5384.69669874 E_{\mathrm{h}}$

Co $0.01018030535352 \quad 0.02308702390101 \quad-0.05035688908211$

Co $-0.01915781347567 \quad 0.03555299304410 \quad 2.46266536986449$

$\begin{array}{llll}\text { P } & -0.24144358497033 & 1.11338905294848 & -1.98399484093811\end{array}$

$\begin{array}{llll}\text { P } & 0.35800820005545 & -2.21364545065202 & 2.79904049074499\end{array}$

$\begin{array}{llll}\text { P } & -0.53845221452695 & 2.95839927915732 & 1.60572877798469\end{array}$

N $1.93294335255663 \quad 0.07395529291141 \quad-0.04371186361891$

N $0.32857199163807 \quad-1.58801546274503 \quad-0.85037057561734$

N $-1.79474636128412 \quad-0.65175588870006-0.07192499632674$

$\begin{array}{llll}\text { N } & -2.16224206933242 & -0.17221176606801 & 2.93573795554574\end{array}$

$\begin{array}{llll}\text { N } & -0.10226565120896 & 0.43503830295844 & 4.40595391823388\end{array}$

N $2.07286566864346 \quad 0.52277893821044 \quad 2.97940048451649$

N $-0.23563422445282 \quad 1.40743335422806 \quad 1.24339251758337$

C $2.53934253863312 \quad-0.89957829066834 \quad-0.70163070484266$

C $1.61031917365358-1.89641935176574 \quad-1.20676295820434$

C $1.86409306975998-3.06487987487446 \quad-1.94120004145999$

H $2.88600340948751 \quad-3.32866029574595 \quad-2.23023943935746$

C $0.78819458083915 \quad-3.89399953485769 \quad-2.29833052090893$

C $-0.52182299421829 \quad-3.54672172405237 \quad-1.93197062711001$

H $-1.36350628034543 \quad-4.18744001250321 \quad-2.21330286745923$

C $\quad-0.73553490534943 \quad-2.36740783914352 \quad-1.20200830882197$

C $-1.97805912869632 \quad-1.79397619540087 \quad-0.71223670757201$

C $-2.92326530826048 \quad 0.02740394289134 \quad 0.55455887752712$

H $-3.84133585313376 \quad-0.11199907559364 \quad-0.04177120149962$

H $-2.69314901196523 \quad 1.09760325512455 \quad 0.57667602018645$

C $-3.22587747967339 \quad-0.45685228120288 \quad 1.98057329774552$

$\begin{array}{lllll}H & -4.17443156998018 & 0.02735637266713 & 2.27612526917444\end{array}$

H $-3.41209254711289-1.54869705934296 \quad 1.97685591155777$

C $-2.44260465827336 \quad 0.00363724201056 \quad 4.19188620697990$

C $-1.28898441502129 \quad 0.33297755046051 \quad 5.05818215300931$

C $-1.36132166837026 \quad 0.52922794885350 \quad 6.44852111844570$

H $\quad-2.31669004672685 \quad 0.45893425242165 \quad 6.97617552115279$

C $\quad-0.19072649780435 \quad 0.81503807375958 \quad 7.16190570731134$

C $1.02981171680041 \quad 0.87669117633767 \quad 6.47788354629243$

H $1.95337540462103 \quad 1.08088592034671 \quad 7.02586440790999$

C $1.04789851591608 \quad 0.67601878159745 \quad 5.08745796831031$

$\begin{array}{llll}\text { C } 2.26444428621803 & 0.72791605949606 & 4.24682768226495\end{array}$

C $3.17698567176813 \quad 0.66570424383195 \quad 2.03795576910869$

H $3.71978968115276 \quad-0.29756998211362 \quad 1.97735578231947$

H $3.90111437630130 \quad 1.42772796959211 \quad 2.38088525661118$

$\begin{array}{llll}\text { C } 2.72206812114690 & 1.09695073406668 & 0.63442964333508\end{array}$

H $2.10642723110469 \quad 2.00236092023137 \quad 0.71860131663162$

H $3.62396670094868 \quad 1.348283058346620 .04914340666229$

C $4.02164463029042-0.98980818803003 \quad-0.91070146883113$

H $4.29085572914861 \quad-1.89470267754313 \quad-1.47992916197667$

H $4.39059659046030-0.11017884272080-1.47636959968913$

H $4.57151523026097-1.015789846500890 .04866071628630$

C $-3.30857846994369-2.45486394436813 \quad-0.91187904900619$

H $-3.21329132158441 \quad-3.36346569589911 \quad-1.52860399567697$

H $\quad-3.76069162259459 \quad-2.75003879312287 \quad 0.05465212964176$

H $-4.02080138185903 \quad-1.77324269645103 \quad-1.41756201689547$

C $-3.83351756124586 \quad-0.10504810656398 \quad 4.75691410197914$

H $\quad-3.84577829339614 \quad-0.13122817868389 \quad 5.85818962815486$ 


\begin{tabular}{|c|c|c|c|}
\hline & 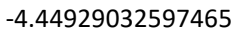 & & \\
\hline & & & \\
\hline & 77 & 6 & \\
\hline & 748 & 70 & \\
\hline & 13 & & \\
\hline & 87074606882838 & 313 & 276 \\
\hline & .77648509088080 & & \\
\hline & 86472 & 2.822184 & \\
\hline & 614431 & 076 & \\
\hline & 17489 & 937 & \\
\hline & 15160214145973 & 621 & -2.4 \\
\hline & .04489750626005 & 5582 & -2.5 \\
\hline & 73 & 50 & -3.3 \\
\hline & 6352 & 46 & -1.6 \\
\hline & 003178 & 163 & \\
\hline & 01 & 0.60898 & -3.4 \\
\hline & 31 & -0.7 & -3 \\
\hline & 4 & & \\
\hline & & & \\
\hline & 0.888 & -3.36 & 1.0 \\
\hline & 942759 & 897 & 2.4 \\
\hline & 35 & 38 & \\
\hline & & & \\
\hline & & 8 & \\
\hline & -0.6 & -2.3 & 5. \\
\hline & & 5 & 4.7 \\
\hline & & & \\
\hline & & -2.7 & \\
\hline & 2.77 & -2.3 & 2.7 \\
\hline & & -3.9 & 65 \\
\hline & & & \\
\hline & & & 0 \\
\hline & -2.8 & & $0 . \varepsilon$ \\
\hline & -2.7 & 31 & 2 \\
\hline & & 3.4 & \\
\hline & & & \\
\hline & & & 17 \\
\hline & 1.28 & 66 & 537 \\
\hline & 0.098 & 2 & 054 \\
\hline & & & \\
\hline & & 4.09 & \\
\hline & -0.34 & & \\
\hline & -0.22774738764241 & & \\
\hline & 97177269430055 & 1.81240008957758 & 8670070378800 \\
\hline
\end{tabular}

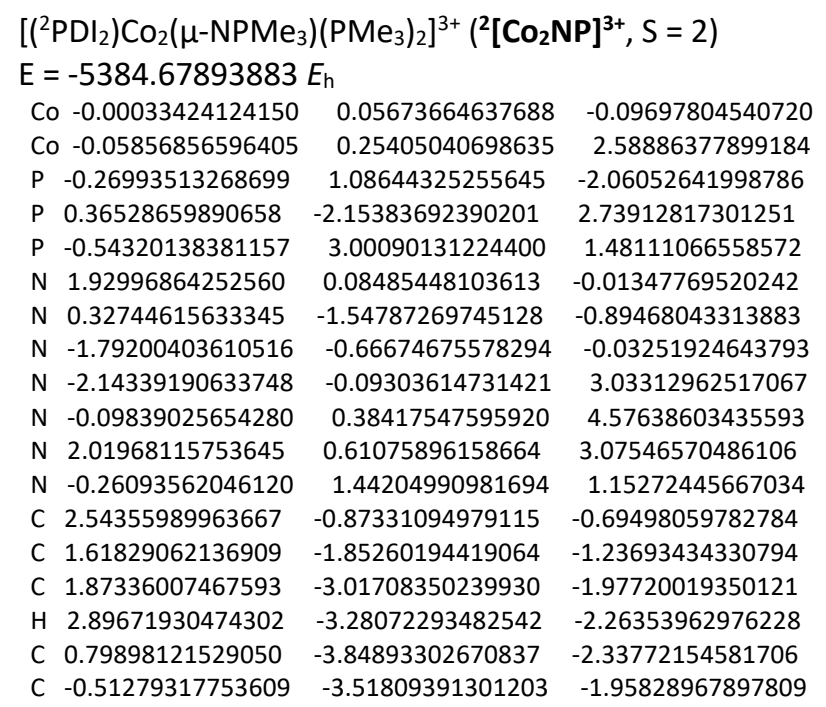




\begin{tabular}{|c|c|c|c|}
\hline & -1.34854144609151 & -4.17123753846975 & \\
\hline & -0.73676225144069 & -2.34370549532515 & -1 \\
\hline & -1.97577630736843 & -1.79884160585619 & 列 \\
\hline & -2.91392288989166 & 0.01178078569667 & 1692727381 \\
\hline & -3.84899313020842 & -0.15618607454876 & 28 \\
\hline & -2.70007705769390 & 1.08587196510630 & ב \\
\hline & -3.19629315911931 & -0.41296213149704 & 168 \\
\hline & -4.14425914893240 & 168291104 & (7) \\
\hline & -3.38940749754431 & 8388978657 & \\
\hline & -2.43022737889399 & 015 & \\
\hline & -1.28914370743119 & 6996 & 1332 \\
\hline & 578667 & & \\
\hline & 557086 & & \\
\hline & 51640 & & \\
\hline & & & \\
\hline & 261 & & \\
\hline & & & \\
\hline & & & \\
\hline & 44 & & \\
\hline & & & \\
\hline & 54 & & \\
\hline & 756 & & \\
\hline & 2.1 & & \\
\hline & 3.63 & & \\
\hline & 4.02 & & 719 \\
\hline & $4.3 \mathrm{C}$ & & -1. \\
\hline & & & 513 \\
\hline & 4.55 & & 351 \\
\hline & -3.3 & & \\
\hline & & & \\
\hline & -3.7 & & \\
\hline & -4.0 & 954 & \\
\hline & 082 & 377 & 663 \\
\hline & -3.80 & & \\
\hline & -4.4 & & \\
\hline & -4.22 & & 372 \\
\hline & & 0.85 & \\
\hline & 3.64 & & \\
\hline & 4.3637 & & \\
\hline & 3.9462 & & \\
\hline & -1.7468 & & -2.2 \\
\hline & -1.73939542321820 & 2.95897789966867 & -1.46 \\
\hline & -1.79064365752891 & 2.58887577260762 & -3.22 \\
\hline & -2.64 & 3274 & 462 \\
\hline & 1.16 & 2.09 & -2.5 \\
\hline & 2.0 & & -2.6 \\
\hline & 0.95 & & -3.5 \\
\hline & & & -1.7 \\
\hline & -0.4 & 12 & 612 \\
\hline & & & \\
\hline & & & \\
\hline & -0.6 & & \\
\hline & & & \\
\hline & & & \\
\hline & & & \\
\hline & & & \\
\hline & & & \\
\hline & & & \\
\hline & & & \\
\hline & & & \\
\hline & & -2.83 & \\
\hline & & & \\
\hline & 2.793 & & \\
\hline & 3092040 & -3.89424 & 2.4626 \\
\hline & -2.302253 & 3.4323 & 1.53 \\
\hline & 2.39513553881140 & 4.4897627245676 & 1.84565492 \\
\hline
\end{tabular}




$\begin{array}{clcc}\text { H } & -2.77385901941195 & 3.30777171909051 & 0.54368414739590 \\ \text { H } & -2.80914520471768 & 2.78823883302984 & 2.27438780066517 \\ \text { C } & 0.08176111741495 & 3.46293444763831 & 3.11594185631307 \\ \text { H } & -0.10134737017383 & 4.54192512251764 & 3.27249965443896 \\ \text { H } & -0.44831390043255 & 2.89394189131973 & 3.89929910469007 \\ \text { H } & 1.16463190477855 & 3.26026212220363 & 3.17148857907815 \\ \text { C } & 0.22876130311537 & 4.18819738393621 & 0.35380556405408 \\ \text { H } & -0.01692589060552 & 5.20509431152353 & 0.71302877738438 \\ \text { H } & 1.32570003305485 & 4.06223516929826 & 0.36251629218464 \\ \text { H } & -0.15435585022969 & 4.07485922307255 & -0.67297736439048 \\ \text { H } & -0.23138422651922 & 0.98514893425990 & 8.37271497789184 \\ \text { H } & 0.98649570501026 & -4.76349427320315 & -2.91169316111675\end{array}$

$\left[\left({ }^{3} \mathrm{PDI}_{2}-\mu-\mathrm{NH}\right) \mathrm{Co}_{2}\left(\mathrm{PMe}_{3}\right)_{2}\right]^{3+}\left({ }^{3}\left[\mathrm{Co}_{2} \mathrm{NCH}\right]^{3+}, \mathrm{S}=0\right)$

$\mathrm{E}=-5002.30092883 E_{\mathrm{h}}$

Co $2.66945320687028 \quad 13.76911606077325$

Co $2.66425788439265 \quad 15.22162193538148$

P $2.70958587932076 \quad 17.14467304945158$

P $2.71464255390906 \quad 13.32869674314751$

N $4.59531172200904 \quad 15.11858028953216$

N $2.71602905635589 \quad 12.08518291413303$

N $0.83997284374441 \quad 13.46144352618655$

N $0.83047544309965 \quad 15.08868577377356$

C $2.74339689276278 \quad 12.03047585135123$

N $4.60349016679972 \quad 13.53272016483150$

C $1.52111844145117 \quad 10.21270378571271$

H $0.57872904683754 \quad 9.70286242915049$

N $2.71146026284877 \quad 13.99505176244626$

C $1.52148261625685 \quad 11.47776267010352$

C $3.91863770464059 \quad 11.51072428274315$

C $1.51174628263815 \quad 12.53174887795192$

H $0.56562266109670 \quad 12.14614173589487$

C $2.75335220746991 \quad 9.60843538045940$

C $5.53346825946614 \quad 15.98332340119288$

H $6.20160425003157 \quad 16.50587859367531$

H $4.93091158495819 \quad 16.74746769070071$

C $3.91352038364614 \quad 13.56088279017955$

C $0.07677701491541 \quad 14.56367186310399$

H $0.20200303632661 \quad 14.56318722649013$

H $-1.00373376783366 \quad 14.48717697434710$

C $0.07828542974285 \quad 15.99486915349135$

H $0.21943226796677 \quad 17.02591977943201$

H $\quad-1.00490205353745 \quad 15.79241581908895$

C $3.96445458546328 \quad 10.24865376268351$

H $4.92435302063884 \quad 9.77397748668717$

C $1.51746241970211 \quad 13.53691120213871$

C $0.40119412691357 \quad 14.23931478006379$

C $5.00583079778220 \quad 14.29560702788296$

C $3.95589708843372 \quad 12.55038769575643$

H 4.9151072440208912 .17119933387721

C $5.54384951551130 \quad 14.51344252069337$

H $4.94278682377346 \quad 15.25417686876456$

H $6.21819195553946 \quad 14.02249497756130$

C $1.35998622454616 \quad 12.22174456420982$

H 0.3702347012085512 .68406296546786

H $1.48805180923741 \quad 11.98049397364875$

H $1.41579344828597 \quad 11.28772219869725$

C $2.62455741700490 \quad 14.78904728277120$

H $3.49636914149147 \quad 15.43961589139638$

H $2.63333395013768 \quad 14.47025255935190$

H $1.70023200293382 \quad 15.35825695674555$

C $5.01162284456310 \quad 12.36425385943881$

C $6.41652544380673 \quad 14.11547342902636$

H $7.08727235646466 \quad 14.92633287113457$

H 6.4380059103433914 .07092213514208
8.43738865093608

6.37454099972289

5.20483680139720

10.64568401530313

6.16017535337008

7.74450959321298

8.40224310543141

6.09563862285728

3.11502112122972

8.41069751815193

6.87275952727085

6.64654359402907

5.03034464406243

7.47808959684915

7.49305035664246

3.57652408839146

3.18164256164732

6.56270514829163

6.89056142116728

6.18170453440781

7.40773475935255

4.57648367990255

8.96255537278719

10.05851735450404

8.75817045422841

6.94899100825726

6.58462727592794

6.94874925413498

6.88109633708718

6.65191418262994

4.55364148435253

5.19270738584336

5.21787316391545

3.60299448988498

3.23480423021444 8.97306171736635

9.52557201121128

9.69738499982988

11.16842707491175

11.02263691712936

12.24067137949569

10.57905961572483

11.73060870352678

11.53494630489979

12.79110942855110

11.52633534240594

7.96301741204126

4.74491717587723

5.07132526810878

3.63937082100436 

H 6.83617643033111
13.15650036543667
C $-1.02161308812970 \quad 14.00206473303665$
H $-1.73411982690391 \quad 14.53193714839003$
H $-1.25177885759449 \quad 12.91925476882818$
H $-1.18869522597348 \quad 14.34263315082211$
C $0.40768168513591 \quad 12.32211553004073$
C $4.17482640993352 \quad 12.40781485129003$
H $4.32476067706316 \quad 11.50683904958539$
H $3.99797556801414 \quad 12.09760089626857$
H 5.0779203939619913 .03769294286206
C $6.41681084239394 \quad 11.84293956738360$
H $6.80109547438852 \quad 11.74732899620734$
H $6.43267867218820 \quad 10.82652055346322$
H $7.11574269308158 \quad 12.47557093564747$
C $-1.02566861362612 \quad 11.90040838033714$
H -1.3299491170821211 .43698521782039$
H $-1.18208325156529 \quad 11.15039863246235$
H $-1.69990471325495 \quad 12.75488503046018$
C $4.14602079264643 \quad 17.33961326644554$
H $5.07437756053637 \quad 17.47783878894588$
H $3.98410142154846 \quad 18.22963453386246$
H $4.24014850764650 \quad 16.44695590048058$
C $2.68546753794423 \quad 18.66206740001530$
H $1.78261481659130 \quad 18.68157470911361$
H $2.69153155641456 \quad 19.55196881290721$
H 3.5789456514018918 .67919299600995
C $1.33205217398376 \quad 17.30072075213497$
H $1.35716508358562 \quad 16.43621189323034$
H $1.47059766862342 \quad 18.23073986812776$
H $0.35145759808478 \quad 17.33771653361845$
C $0.69118120700894 \quad 15.83481885266302$
H $0.45053622591626 \quad 16.71088818082377$
C $6.37938820248136 \quad 15.22692223756837$
H 7.0263352004589215 .96181609953390
H $7.04994810088221 \quad 14.51278328890962$
N $2.19486334497546 \quad 15.66255945141093$
H $2.67970933609223 \quad 16.39969119852303$
H $2.76903141545134 \quad 8.62480110362885$
H 2.75783633937480
5.11098304424833
4.80315912142258
5.45651371539727
4.82939284478041
3.76070168796005
7.91379143917459
11.23253457645063
10.61032862799705
12.27982831866466
11.19582318833459
7.96630401930938
6.93106108090712
8.40558050277109
8.53464864162766
7.85075570671451
8.81285313780505
7.05542331446693
7.66439893592166
4.09833821655425
4.67478097167345
3.46131811504895
3.45346689891347
6.21219374399626
6.84836083517857
5.55318906032698
6.86247898407220
4.01569344372892
3.32661159417665
3.43249736658663
4.51709371558803
8.35076793533160
8.97250141664506
7.91228361138555
8.42817265561703
7.40277056561159
8.22764107392898
8.75032301046754
6.07964287821736
2.36026614351795

${ }^{3} \mathrm{TS}_{\mathrm{NN}}(\mathrm{S}=0)$

$\mathrm{E}=-5111.69988835 E_{\mathrm{h}}$

Co $-0.00476177734108 \quad-0.06931985657458 \quad 0.02573594973923$

Co $0.00749451544558 \quad-0.04092625951474 \quad 2.50194943942880$

$\begin{array}{llll}\text { P } & 0.04647800570257 & -1.03434309252315 & -2.02793735911410\end{array}$

P $0.06070275958291 \quad-0.95385638960901 \quad 4.57864216362634$

N $-1.95035153385855 \quad 0.11188121440430 \quad-0.13578472657093$

N $-0.10300427870261 \quad 1.72987850932296 \quad-0.42441117002111$

N $1.89393750324893 \quad 0.30277601662526 \quad-0.13731844257391$

N $1.91032182937982 \quad 0.33768744733955 \quad 2.64547008633494$

N $-0.09083211429444 \quad 1.76514431847770 \quad 2.92470358303553$

N $-1.93801387230055 \quad 0.14051067488571 \quad 2.67444082517436$

N $\quad-0.17436761091339 \quad-1.36736303695023 \quad 1.28013398910640$

N $0.70433075927796 \quad-2.77093875502477 \quad 1.29847794533811$

N $0.58823609285488 \quad-3.88483689198755 \quad 1.32395842534618$

C $-2.38843862468706 \quad 1.31130590441202 \quad-0.43655151382581$

C $-1.32319169742541 \quad 2.30898905209973 \quad-0.53498523988242$

C $-1.42286894922916 \quad 3.69417801369743 \quad-0.73162288806882$

H $-2.40212490879659 \quad 4.17697395080806 \quad-0.81378871588911$

C $-0.24024598909375 \quad 4.44988959715924 \quad-0.81763420676405$

C $1.01212106048462 \quad 3.81613047757350 \quad-0.74230811267581$

H $1.93736038108370 \quad 4.39412467600196 \quad-0.83220646011244$

C $1.05332519907434 \quad 2.42739004629176 \quad-0.54432406409321$

C $2.21098635977620 \quad 1.54143250798933 \quad-0.44572795640328$

C $2.92080312408634 \quad-0.74213755352635 \quad-0.02331210703826$ 
H 3.59526089486887

H 2.40569483411031

C 3.75483030268350

H 4.46709911491145

H 4.36039043449409

C 2.94063930871267

H 2.42962606789883

H 3.62830884608490

C 2.22421196453341

C 1.06462234723007

C 1.02387633152310

H 1.94972205758910

C -0.22864837749914

C -1.41082484852378

H -2.39007664359942

C -1.31085260758258

C -2.37537657779691

C -2.84679523787161

H -3.52243388690959

H -2.22119436395828

C -3.68345319642855

H -4.38054625404571

H -4.30885935426265

C -2.86077235134587

H -2.23788815438663

H -3.54541910579654

C -3.80876649758574

H -3.85214826274257

H -4.20527455517814

H -4.48011954976524

C 3.58702379389421

H 3.95797625955001

H 3.56112495877098

H 4.32133847578676

C 3.59389669014405

H 4.36683608819053

H 3.57720515965533

H 3.89888013791995

C -3.79343639710176

H -4.19900753914860

H -3.82885890404326

H -4.46260945514165

C -1.42206040887223

H -1.60156806848410

H -2.31146187454415

H -1.23507341296940

C 0.21754241087276

H -0.61389374792607

H 1.17794173970361

H 0.19549909938442

C 1.39772125344165

H 1.25825602875773

H 2.37681031025811

H 1.36978252175222

C 0.12237607059668

H 1.06824871423333

H -0.72869428609068

H 0.06926275555873

C 1.46268179639722

H 1.31972150544963

H 1.49258937141296

H 2.41638792707316

C -1.36581676738522

H -1.18690956444465

H -2.29337924623454

H -1.47313622159442
$-0.70332451724540$

$-1.71315217723197$

$-0.63661047813064$

$-1.48402244113503$

0.28691144906635

$-0.70646951339574$

$-1.67825057479114$

$-0.64075855978874$

1.58086740659933

2.46549528841767

3.85584472638049

4.43438014226305

4.48916236586831

3.73124765289199

4.21388228544254

2.34411385078825

1.34368317919040

$-1.01103605421928$

$-1.03724502219449$

$-1.91584758466164$

$-1.00805201241547$

$-0.15010399427360$

$-1.92134364269536$

$-1.03541173099378$

$-1.94239868921744$

$-1.07429091750856$

1.69815247647597

2.30277332649166

2.33584973238804

0.83316534834785

2.06231787300600

2.66488705573089

2.73415522979806

1.26464020247634

2.11626611721223

1.33616584020266

2.67984113805501

2.83503634912023

1.73184542192870

2.36053543627626

2.34589090708601

0.86744532915282

$-0.68886229550903$

0.40062403185243

$-1.19493584086072$

$-1.06286720262627$

$-2.84560809708828$

$-3.30972598493640$

$-3.13988592089465$

$-3.19998052320002$

$-0.41416117912435$

$-0.80464881097043$

$-0.74980327486199$

0.69092369031227

$-2.77065295852083$

$-3.13187810144279$

$-3.20147346200564$

$-3.09434283774735$

$-0.38993396014028$

$-0.75596509771822$

0.71519517380334

$-0.78232684868451$

$-0.48479196764393$

$-0.83672857290631$

$-0.94334605492438$

0.61531270712967
$-0.89806507188814$

$-0.05064093082672$

1.25256718500328

1.25920116104644

1.23248603308724

2.54439767374321

2.60634029550074

3.40670130551382

2.93683064720924

3.02964654592959

3.21432896474213

3.29324116048449

3.29118883800548

3.21993960631253

3.30356786503981

3.03684944279580

2.96102499431226

2.56865956808129

3.44292125159284

2.59476285507776

1.28853015834370

1.28430492935278

1.30060563601637

$-0.00021465219801$

$-0.01735371370336$

$-0.86670567287420$

$-0.71495856501681$

$-1.64151501681737$

0.10117001417195

$-0.83008306147691$

$-0.72463634537918$

0.12934324580884

$-1.60357639303530$

$-0.92000873987772$

3.22016354900197

3.29195827544752

4.17376164985697

2.43305806343678

3.24879613300242

2.43005239159419

4.16957483842228

3.37958196145092

$-3.05080537543997$

$-3.09172813331568$

$-2.64340527853200$

$-4.07513063496333$

$-2.05313679108087$

$-1.49256605064411$

$-1.59452343151580$

$-3.10192006567458$

$-3.08265060045478$

$-4.10847620257207$

$-2.70486989755055$

$-3.10871898530740$

4.65251138296969

4.21220043287851

4.09512911271804

5.70997238499937

5.59846868392402

6.63282455993844

5.60685411985843

5.21102829435076

5.61240758339066

6.64597349427273

5.23471447695535

5.61834559900669 
$\begin{array}{llll}\text { H } & -0.28433624169229 & 5.57571409340846 & 3.42298595853306\end{array}$ H $-0.29531884584766 \quad 5.53515350316321 \quad-0.95964115713221$

${ }^{2} \mathrm{TS}_{\mathrm{NN}}(\mathrm{S}=0)$

$\mathrm{E}=-5033.22425136 E_{\mathrm{h}}$

Co 0.00576895285824

Co $0.00143044638587-0.02354924462321$

N $0.01225525949479 \quad 1.29686067596307$

$\begin{array}{lll}\mathrm{N} & -1.04572702934171 & 2.56984143447011\end{array}$

N $-1.07518113138312 \quad 3.68968513686511$

P -0.16199084486677

P -0.16825280801677

N 1.95287247023195

N 0.30946754559498

N -1.82711714589183

N -1.83280942178466

N 0.29746832399822

N 1.96067760859312

C 2.53536991845850

C 1.58458472438670

C 1.82480936823707

H 2.84468540613752

C 0.73123290105651

C -0.57748278394371

H -1.43642784716524

C -0.76248345621796

C -2.01052901335074

C -2.91600555560551

H -3.88947048490389

H -2.76048873390029

C -2.91936906944442

H -2.76508125941421

H -3.89460391339276

C -2.02020946175517

C -0.77492926863742

C -0.59024555317170

H -1.44931733090606

C 0.71771862415220

C 1.81169715834522

H 2.83423392637183

C 1.56792257405763

C 2.53037705742730

C 2.72411469614322

H 3.75400313187179

H 2.23295536242552

C 2.73584771019551

H 2.27723846433263

H 3.77165564474306

C 4.01838103888716

H 4.44139664240944

H 4.26539715399395

H 4.53445567782531

C -3.31201742750537

H -4.17906319255830

H -3.25928277649027

H -3.49432996636052

C -3.32306028466683

H -3.51069810825172

H -3.26808438119373

H -4.18729466626947

C 3.99574525970009

H 4.63586452816115

H 4.27698473926602

H 4.22066296585423

C -1.80012137465713
1.05113208298875

1.07516157855497

$-0.01092141896581$

$-1.75919702122951$

$-0.64741169019526$

$-0.63610949895034$

$-1.74291010589291$

$-0.00485960733691$

$-1.10880152423583$

$-2.16644559640820$

$-3.46499291750429$

$-3.81148211895165$

$-4.31695547039113$

$-4.52393963671135$

$-2.56110455748833$

$-1.88308071801701$

0.17706583715010

$-0.16238604351129$

1.20227726000070

0.18412009731213

1.21235610663658

$-0.15164909080806$

$-1.86646866478651$

$-2.54186221920004$

$-3.84203204016461$

$-4.49574804585872$

$-4.28627369125874$

$-3.43662086081955$

$-3.77179842454327$

$-2.14789424394741$

$-1.10143075468135$

1.12124687228181

1.12326599297265

2.04258112562560

1.10589957072773

2.03584342349335

1.06765715286612

$-1.29005608834492$

$-0.55843999519975$

$-2.30067743879939$

$-1.14189731071210$

$-2.61308073587055$

$-2.04762028411177$

$-3.57033748102881$

$-2.86819069702836$

$-2.59303016131915$

$-2.83383871137665$

$-3.55765003761068$

$-2.03165766580912$

$-1.35989656936734$

$-0.53925907689140$

$-2.28646042554830$

$-1.53517788794409$

1.72516743653178
$-3.86850954966711$
0.07715240706153

2.52590846893575

1.29654651184711

1.29055577015457

1.28620715633906

$-1.88378912241671$

4.47924723456019

$-0.00124209439171$

$-0.53947574745076$

0.00549344653680

2.59662188030687

3.16457526103243

2.61345928701545

$-0.43313096172849$

$-0.76802064015017$

$-1.24470290786538$

$-1.43827372520837$

$-1.47372494052934$

$-1.22868312213634$

$-1.40451571297039$

$-0.75484178512843$

$-0.40971422506695$

0.51476480357097

0.13133935535511

0.15581212955694

2.07532659277322

2.42630261511527

2.45775543867816

3.02605730463650

3.38649466736115

3.88097397917876

4.06271450000125

4.13960008968147

3.90160312706842

4.10324799495085

3.40404715793929

3.05982755651305

2.08334770817882

2.46934547401540

2.41533728155670

0.51873877675740

0.16372046472294

0.15012071494107

$-1.27858847778686$

$-0.92527679654699$

0.40447202368781

$-0.53367342978244$

$-0.16132819550334$

0.01911296442483

$-1.59689671414358$

3.15318197136901

4.21883946723097

2.61348396535425

2.76866888126300

3.22295144625756

2.86682538909242

2.68534543618075

4.29455097093911

$-2.30289278932392$
$-0.56230842013732$ 


$\begin{array}{llll}\text { H } & -1.74678789727905 & 2.17371559368477 & -3.31372994160518 \\ \text { H } & -2.09125806860188 & 2.50718032959381 & -1.57963446929523 \\ \text { H } & -2.55503879568331 & 0.91923158968991 & -2.30514920173333 \\ \text { C } & 0.22466068787884 & -0.07613693369016 & -3.25743458454371 \\ \text { H } & 0.17442217612400 & 0.47595572803265 & -4.21547926030247 \\ \text { H } & -0.50394507714969 & -0.90705350215067 & -3.27658559004186 \\ \text { H } & 1.24183617933405 & -0.48726559487008 & -3.128685666582955 \\ \text { C } & 0.96601810552853 & 2.45989362065235 & -2.10324118071769 \\ \text { H } & 2.01228265643673 & 2.10910266923435 & -2.10104243295181 \\ \text { H } & 0.81870743040859 & 3.19859884605244 & -1.29405074727928 \\ \text { H } & 0.74946985325893 & 2.94250773092386 & -3.07622924770240 \\ \text { C } & -1.81336361594172 & 1.73044955879083 & 4.90055058085395 \\ \text { H } & -2.55856182004938 & 0.91552778612775 & 4.90764585771226 \\ \text { H } & -2.11547235924174 & 2.50616186889384 & 4.17488670431750 \\ \text { H } & -1.76300875014089 & 2.18387920855130 & 5.90937976290536 \\ \text { C } & 0.24271894826427 & -0.03194242917410 & 5.86268615123851 \\ \text { H } & 0.19557299549702 & 0.53127030870136 & 6.81436130983995 \\ \text { H } & 1.26321531849488 & -0.43356406163813 & 5.72979991166091 \\ \text { H } & -0.47661094676726 & -0.87020900204055 & 5.89889038816059 \\ \text { C } & 0.94047177626121 & 2.50224204947957 & 4.67799991333551 \\ \text { H } & 0.72366325583072 & 2.99048495003413 & 5.64817026326682 \\ \text { H } & 0.77599700357900 & 3.23092501457634 & 3.86311676651434 \\ \text { H } & 1.99180036957032 & 2.16709974747021 & 4.67133907126636 \\ \text { H } & 0.88514474441879 & -5.29638669798694 & 4.52889605139401 \\ \text { H } & 0.90047883941953 & -5.33302202484996 & -1.84640627433832\end{array}$

${ }^{3} \mathrm{TS}_{\mathrm{NP}}(\mathrm{S}=0)$

$\mathrm{E}=-5002.18963269 E_{\mathrm{h}}$

Co $0.02883279835924 \quad 0.04124845831260 \quad 0.32832132221040$

Co $-0.05215495415341 \quad 0.44514580621124 \quad 2.82845249903501$

$\begin{array}{llll}\text { P } & 0.13625180943987 & -1.30328598782801 & -1.46203019243093\end{array}$

$\begin{array}{llll}\text { P } & 0.02248473799445 & -1.73446518075987 & 3.61873059613775\end{array}$

N $-1.90831672623634 \quad 0.17599163059845 \quad 0.17806518671294$

$\begin{array}{llll}N & -0.10134803504828 & 1.75079513713438 & -0.44823967529892\end{array}$

$\begin{array}{llll}\text { N } & 1.95599581032240 & 0.47348648655427 & 0.09159235727655\end{array}$

N $1.92756542122333 \quad 0.91147968887885 \quad 3.11132801695050$

$\begin{array}{llll}N & -0.14462613031755 & 2.29855602809184 & 3.17704036259291\end{array}$

$\begin{array}{llll}\text { N } & -1.99809385710974 & 0.67089986036116 & 2.98496854859616\end{array}$

N $0.41858155133190 \quad-0.89273615503603 \quad 1.79168027541555$

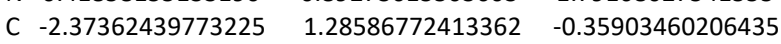

C $-1.33777373161149 \quad 2.26756586862233 \quad-0.65092118169497$

C $-1.47967951357202 \quad 3.59005936126619 \quad-1.10268165011504$

H $-2.47404828553684 \quad 4.01969003996551 \quad-1.26126138476586$

C $-0.32355913055836 \quad 4.35007320374410 \quad-1.33986042772249$

$\begin{array}{llll}\text { C } & 0.94746607529733 & 3.77698284766588 & -1.15356186940926\end{array}$

H $1.85550968544996 \quad 4.35521970671642 \quad-1.35076678807217$

$\begin{array}{llll}\text { C } & 1.02711028700954 & 2.45173229036472 & -0.70234091608110\end{array}$

$\begin{array}{llll}\text { C } 2.21702243412279 & 1.64976597284340 & -0.42695820317596\end{array}$

$\begin{array}{lll}\text { C } 3.04567759930409 & -0.43948935256527 & 0.46257576953893\end{array}$

H $3.73311774520985-0.55584252434949 \quad-0.39477413114921$

H $2.59649087830024 \quad-1.42266278352403 \quad 0.66893032690061$

$\begin{array}{llll}\text { C } 3.83823595608899 & 0.02873066859112 & 1.68677940658007\end{array}$

H $4.69569630716948-0.66208117577435 \quad 1.80083868853750$

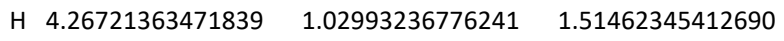

C $3.04750108589968 \quad-0.02971579203410 \quad 2.99511367904683$

H $2.63366049017890 \quad-1.03208408050363 \quad 3.06060441831304$

H $3.72677728861077 \quad 0.10742935441149 \quad 3.85641754299099$

C $2.18152408565329 \quad 2.19240426450046 \quad 3.23734343089284$

$\begin{array}{llll}\text { C } & 0.99469370373817 & 3.03526693051494 & 3.21042856315871\end{array}$

$\begin{array}{llll}\text { C } & 0.94633486391892 & 4.43459436487483 & 3.14653475601442\end{array}$

H $1.86938473682208 \quad 5.02188367605941 \quad 3.17459601470950$

C $-0.30275902940874 \quad 5.06732704661847 \quad 3.02587706984642$

C $-1.47151522073052 \quad 4.29309822075854 \quad 2.99164781792785$

$\begin{array}{llll}\text { H } & -2.45418965255120 & 4.76736407643453 & 2.89717042180885\end{array}$

C $-1.36293055512706 \quad 2.89578996517313 \quad 3.07150040573077$ 
C -2.43070320412403

C -2.98726588011154

H -3.72036674277474

H -2.47697537979671

C -3.72319738513312

H -4.37925529873578

H -4.38576666047401

C -2.82075302411484

H -2.20007530737742

H -3.46148521406949

C -3.79882339731021

H -3.82967168652180

H -4.26578568158708

H -4.42282369217937

C 3.57376566278366

H 3.87579742604413

H 3.53735845651233

H 4.35610761087816

C 3.53656158256315

H 4.32782389606917

H 3.50421588308343

H 3.82408204310886

C -3.85880541902664

H -4.20838123261532

H -3.94144034552135

H -4.55101346438785

C -1.33036797175045

H -1.57185060929854

H -2.19514851161730

H -1.10549734237071

C 0.37755728067560

H -0.50845847206109

H 1.27113068806752

H 0.50934632620500

C 1.48606638431105

H 1.37543503734714

H 2.46694269501554

H 1.42529158523527

C -0.84311627662205

H -0.15681833198172

H -1.78895219503174

H -1.05125768909143

C 1.54790111869220

H 1.18881557265636

H 2.15203667063264

H 2.14150138658616

C -0.75373757032758

H -0.90464389094020

H -1.70861452974230

H -0.03370885077479

H -0.36308513399085

H $\quad-0.41075068147524$
1.91603655310031 $-0.41785905524318$ $-0.22083762436632$ $-1.34032316156762$ $-0.64261640734331$ 0.21633654735300 $-1.51899562636250$ $-0.93055339069086$ $-1.81957500623442$ $-1.14880490000397$ 1.56790542266699 2.06385306812169 2.26705639002702 0.66171528286205 2.19582629318349 2.95598372917125

2.70509478312202

1.42140535695247 2.82620735777985

2.10781263711082 3.61056089188118 3.32689623865854 2.36221287202234 2.78363500790496 3.17086655551964 1.55535430394483 $-1.27304905864525$ $-0.23017249183338$ $-1.73485317924023$ $-1.84628998471786$ $-3.04936506164957$ $-3.40910832957245$ $-3.14673749729050$ $-3.65516127006278$ $-0.89143311147929$

$-1.50788052957905$ $-1.10052567185358$ 0.17665233887499 $-3.14633939074119$ $-3.58323976868486$ $-2.88540196023212$ $-3.88856044885536$ $-2.58312467154993$ $-3.47373871474708$ $-1.97015704624508$ $-2.92523326476031$ $-1.49599205264128$ $-2.49254089676499$ $-0.95245091268035$ $-0.91072215206500$ 6.16004121624787 5.38734177640894
3.06283410521289 3.02131433849604 3.82297604329748 3.30782335397403 1.70295218684533 1.48075705985081 1.83982726237473 0.50112416122273 0.68029467088378 $-0.37152062445411$ $-0.72923171578179$ $-1.71750940710250$ $-0.00604026495713$ $-0.77818456112700$ $-0.75659727120678$ $-0.00842070561935$ $-1.73796377967243$ $-0.79590560841698$ 3.36398993208035 3.62685469120855 4.14415712867109 2.41666324272887 3.17035859742817 2.20556939350926 3.92090938549307 3.45507861145708 $-2.54006921063484$ $-2.81471595958563$ $-2.03729998380582$ $-3.45962431829027$ $-1.01539958943157$ $-0.46276167879422$ $-0.37278482885023$ $-1.93265433151755$ $-2.61212312665815$ $-3.52413502325209$ $-2.15531190022030$ $-2.88851467119299$ 2.83847825824608 2.09269866829831 2.34424524299117 3.63151241105974 4.13995003141315 4.69215783083502 4.82821274538158 3.27666838579595 5.28569255392265 5.74598533031604 5.25077669836801 5.88556564156651 2.95913079558238 $-1.68110010701071$

${ }^{2} \mathrm{TS}_{\mathrm{NP}}(\mathrm{S}=0)$

$\mathrm{E}=-4923.74375796 E_{\mathrm{h}}$
Co 0.17802930745559
Co 0.04319871805956
N -0.35081127237481
$-0.49946549334314$
$-0.82167511235447$
0.11296468937169 2.71504124015065
P 0.15982978985037
0.44093250186441 1.58703404985935
P -0.51361402804422
1.27428047046018
$-1.21101561453098$
N 2.16432470280735
1.25551873459166
3.35648870874339
N 0.31488458406987
$-0.77595839276722$
0.06791371931441
N $-1.70045220536362 \quad-0.89231218043842$
$-1.25393501063858$
$-0.1909210969267$
$\begin{array}{llll}\mathrm{N} & -1.73911337131810 & -1.62327822956826 & 2.82421794786866\end{array}$
N $0.36668796963776 \quad-2.05968934227166 \quad 4.06939883112993$ 


\begin{tabular}{|c|c|c|c|}
\hline & 1.92655657799169 & -0.51672801889306 & \\
\hline & 2.59738405190721 & -1.56386499034686 & \\
\hline & 54288607585889 & -2.15194867645707 & 468 \\
\hline & 666 & -2.9 & 813 \\
\hline & 65338609732353 & -3.27096011260835 & -3.206135 \\
\hline & 50664723721758 & -3.40003526433180 & -3.50198 \\
\hline & 845605 & -3.009957 & -3.02 \\
\hline & 350873 & -3.332907 & -3.5 \\
\hline & 826264 & 9796 & -1.88220 \\
\hline & 152071 & -1.7019322 & 7778 \\
\hline & 067023 & -0.4 & 0.74 \\
\hline & 684524 & 420 & 5696 \\
\hline & 414451 & 7923 & 5778 \\
\hline & -2.896986 & 116 & 1.9 \\
\hline & -3.758730 & 793 & 2.52 \\
\hline & -3.1 & 202 & 1.5 \\
\hline & 363785 & 938 & 3.7 \\
\hline & -0.681236 & 3217 & 4.57528 \\
\hline & 23566 & 3707 & 5.67 \\
\hline & -1.3 & 422 & 634 \\
\hline & 0.80 & -3.7 & \\
\hline & 1.87 & -2.9 & 27 \\
\hline & 2.88 & -3.0 & 6.0 \\
\hline & 1.61 & -2.1 & 4.5 \\
\hline & 2.52 & -1.2 & 21 \\
\hline & 2.64 & & \\
\hline & 3.45 & 0.9 & 2.71 \\
\hline & 1.92 & 1.2 & 1.8 \\
\hline & 3.20 & -0.1 & 380 \\
\hline & 3.75 & & 58 \\
\hline & 3.93 & -0.5 & 347 \\
\hline & 4.04 & -1.8 & 568 \\
\hline & 4.61 & -0.8 & 277 \\
\hline & & & \\
\hline & & & -0.30 \\
\hline & -3.39 & -2.1 & -1.5 \\
\hline & -4.02 & -1 & -1.7 \\
\hline & -3.86 & & -0.7 \\
\hline & & & \\
\hline & -3.16 & -3.2 & 4.0 \\
\hline & -3.09 & -3.8 & 654 \\
\hline & -3.4 & & \\
\hline & -4.0 & & \\
\hline & 3.97 & -1.1 & 4.25 \\
\hline & 4.458 & -0.2 & 3.93 \\
\hline & 4.51 & -1.9 & 3.76 \\
\hline & 4.11 & -1.2 & 5.342 \\
\hline & 1.581 & 2.3 & -1.004 \\
\hline & 1.448 & 3.2 & -1.66 \\
\hline † & 2.510 & 1.8 & -1.28 \\
\hline & 1.65 & & 0. \\
\hline & -1.312 & & -0.965 \\
\hline & -1.24 & & -1.608 \\
\hline & -1.37 & 5140 & 0.091 \\
\hline & $-2.216 \mathrm{~S}$ & & -1.2399 \\
\hline & 0.15890239005782 & 0.8464589 & -2.97579 \\
\hline & -0.71203 & & -3.21087 \\
\hline $\mathrm{H}$ & 1.089 & 0.3 & -3.230 \\
\hline & 0.101 & & -3.57390 \\
\hline & -0.139287 & 1.068467 & 5.15968 \\
\hline & 0.927887 & 0.82 & 5.29337 \\
\hline & -0.76 & 0.25770 & 5.57827 \\
\hline & -0.36599892180694 & & 5.67678 \\
\hline & 0.34191085506928 & 2.82418022486004 & 2.99779144436268 \\
\hline & -0.15630447263574 & 3.60471075378841 & 3.60395182765448 \\
\hline & 0.26442745 & 3.09119315 & 9572688 \\
\hline
\end{tabular}




$\begin{array}{cccc}\text { H } & 1.39967114276717 & 2.77160471358754 & 3.30653254576393 \\ \text { C } & -2.27513630368511 & 1.68638505891117 & 3.36981794500663 \\ \text { H } & -2.41600231789201 & 2.43147604722102 & 4.17551082789440 \\ \text { H } & -2.89917879741110 & 0.80607017943066 & 3.59369874291058 \\ \text { H } & -2.57535877805464 & 2.13905378067971 & 2.41005354900548 \\ \text { H } & 0.97959882273099 & -4.38507384287430 & 7.07424229294154 \\ \text { H } & 0.58467328912712 & -4.03292816639430 & -4.39322538077457\end{array}$

$\beta-{ }^{3} \mathrm{TS}_{\mathrm{NH}}\left({ }^{3} \mathrm{TS}_{\mathrm{NH}}, \mathrm{S}=0\right)$ : PCET from $\beta-\mathrm{CH}_{2}$ hydrogen to $\mu-\mathrm{N}$ $\mathrm{E}=-5002.22123652 E_{\mathrm{h}}$

Co $2.72747423072500 \quad 15.18517149990847$

$\begin{array}{lll}\text { P } 2.72184852604757 & 17.20433855302199\end{array}$

P $2.73228573309579 \quad 13.49033867158033$

N $4.64275548314151 \quad 15.04983793498984$

N 2.71495173330998 12.02951509328034

N $0.84710070005653 \quad 13.47085973305733$

N $0.83507249545946 \quad 15.05880391591853$

C $2.71098600273005 \quad 11.96569364327930$

N $4.64856912731588 \quad 13.43759033446998$

C $1.47583417319233 \quad 10.21618933022685$

H $0.52227231630070 \quad 9.73763983487238$

N $2.72308597691158 \quad 13.97692328790130$

C $1.51389336871744 \quad 11.46145233667084$

C $3.89877012269234 \quad 11.43850340589937$

C $1.49404603291987 \quad 12.48235363626702$

H $0.54063329365866 \quad 12.09038245277151$

C $2.68906084371785 \quad 9.59210078283444$

C $5.61463964878219 \quad 15.87843709316097$

H $6.28741871756486 \quad 16.38419322780659$

H $5.04284930144628 \quad 16.64959179373653$

C $3.91068093143035 \quad 13.51484315036503$

C $-0.05590782451059 \quad 14.49083445821388$

H $0.07227664377430 \quad 14.52496999715242$

H $-1.11258676645479 \quad 14.21348193546616$

C $-0.05802474522993 \quad 15.93458537976993$

H $0.07821859104636 \quad 16.97194320793695$

H $-1.11909462300670 \quad 15.68132549339581$

C $3.91585388169219 \quad 10.19588841158156$

H $4.86366816033556 \quad 9.70797007615854$

C $1.52802213638976 \quad 13.50800071856085$

C $0.42230606657927 \quad 14.20818171192495$

C $5.02283586219229 \quad 14.23113561101480$

C $3.93395536927095 \quad 12.48855685176521$

H $4.88423213247736 \quad 12.10065714402047$

C $5.62817740033174 \quad 14.39817336634322$

H $5.06334548775227 \quad 15.15728208605571$

H $6.30797153224362 \quad 13.88514543992197$

C $1.44046916372122 \quad 12.32728997350769$

H $0.42509140106509 \quad 12.70507834211814$

H $1.55161536488313 \quad 12.16374318942176$

H $1.58037576115851 \quad 11.36534566475428$

C $2.52021313843608 \quad 15.02041406856081$

H $3.35102249001864 \quad 15.70786041177381$

H $2.53123694808078 \quad 14.79067720603346$

H $1.56663176296351 \quad 15.51465936867335$

C $5.01798104247286 \quad 12.25973434782204$

C $6.42545029644312 \quad 14.02625900460799$

H $7.10948642600152 \quad 14.83568541060611$

H $6.43228339081918 \quad 13.95824973321185$

H $6.83773748762326 \quad 13.07030729066335$

C $-0.99214206006890 \quad 13.95838228226459$

H $-1.15171967773574 \quad 12.87930900068639$

H $-1.19673403147034 \quad 14.48246909920661$

H -1.73700497864673

8.41093651048037

6.39124077898233

5.35822579266701

10.66403054993060

6.09682353889325

7.75472665183731

8.39847854803466

6.10922796637934

3.10466140808108

8.37472751852957

6.82132414644640

6.57759936681612

4.97839841626447

7.46983605937131

7.46266484651103

3.58221280398768

3.21424856668052

6.48343192969821

6.82904840365029

6.11297091331915

7.36647993720687

4.51705252680212

8.94968635509837

10.04503802434525

8.76619096416998

6.87997085280973

6.53166891615422

6.68852248811532

6.81285029674139

6.56388627798465

4.53959388400842

5.19310510782971

5.13979639388788

3.56114970835298

3.18015431093673

8.90957541852942

9.47189870306204

9.61163005121285

11.22307869122190

11.02293041081120

12.31219094284602

10.69680089640754

11.62764087159591

11.38282601799117

12.71101620921470

11.36810331447467

7.92268892437192

4.65359618983279

4.95583071236836

3.54917508654985

5.03623400711374

4.77336151399080

4.59217269930278

3.81677444496439

5.50966533462563 
C $0.42062638430061 \quad 12.32113473354224$

C $4.22871024467920 \quad 12.71889265448639$

H $4.47884722961801 \quad 11.80116894654614$

H $4.02163747573233 \quad 12.45348347363628$

H $5.07993062060048 \quad 13.41721950508056$

C $6.41243936378682 \quad 11.71162672445079$

H $6.77640682808132 \quad 11.62843915978063$

H $6.41483862824544 \quad 10.68802944081509$

H $7.13285757052923 \quad 12.32194600265032$

C $-1.01583999940850 \quad 11.89829493768348$

H $-1.65919617804921 \quad 12.67077896331179$

H $-1.40024833669826 \quad 11.71198542095648$

H $-1.13369112592130 \quad 10.96722509160148$

C $4.21086038597193 \quad 17.59733935396934$

H $5.07432261862162 \quad 17.77621355595655$

H $4.01424738349505 \quad 18.51486062740993$

H $4.43621330067361 \quad 16.76626052891291$

C $2.52291781547060 \quad 18.63455783487684$

H $1.59179538712457 \quad 18.54527906691409$

H $2.49594433840226 \quad 19.57044836349360$

H $3.37836173160123 \quad 18.66131449247299$

C $1.41974497587593 \quad 17.33770616111576$

H $1.54826243776026 \quad 16.51149898822891$

H $1.53242031161480 \quad 18.30333418480233$

H $0.40702490970187 \quad 17.28474425365015$

C $0.21282808729611 \quad 15.83008763403416$

C $6.45400787856728 \quad 15.08236550115738$

H $7.14069287509316 \quad 15.79168618941175$

H $7.08663429015053 \quad 14.34781667104023$

H 2.678059173020898 .62559655906420

H $2.70621528484777 \quad 11.15664626231296$

H $-0.09380269161225 \quad 16.69730517502271$

N $2.65622293447804 \quad 15.57003521125624$

H $1.69170965694793 \quad 16.08298244035256$
7.91807291439316 11.36680621402724 10.80486749239592 12.42062811395017 11.33770541183258 7.89412630973491 6.85039473327005 8.31520246262166 8.45976177959517 7.86394675376450 7.40336600879104 8.88726611000264

7.28585133391399

4.38130842748647 5.04106930043570 3.79468853715821 3.68949119157215 6.46770395441997 7.05528528992702 5.87647575513770 7.16732607922436 4.08419125461135 3.36028734737486 3.55524810957911 4.51521510931408 8.34504563736044 7.82401628469932 8.32509072343146 7.29483050511958 5.96816082124516

2.36504317417625 8.94830292838004 8.16644752346106 8.52792520610054

\footnotetext{
$\alpha-{ }^{3} \mathrm{TS}_{\mathrm{NH}}(\mathrm{S}=0)$ ): PCET from $\alpha-\mathrm{CH}_{2}$ hydrogen to $\mu-\mathrm{N}$

$\mathrm{E}=-5002.20379750 E_{\mathrm{h}}$

Co $-0.10533266261120 \quad-0.01894874749827 \quad 0.06201027762660$

$\begin{array}{llll}\text { Co } 0.04862624536691 & 0.00707865950103 & 2.56118322313038\end{array}$

P $-0.04430870709158 \quad-1.09776456091566 \quad-1.94451436558390$

$\begin{array}{lllll}\text { P } 0.09853025251195 & -0.96792904847012 & 4.58725781653797\end{array}$

N $\quad-2.04441440692629 \quad 0.25687830607865 \quad-0.17393716862281$

N $-0.12976400044187 \quad 1.80785957376300 \quad-0.37546166031403$

N $1.81293104493451 \quad 0.28913473212662 \quad-0.08060400609360$

N $1.96088536295617 \quad 0.43164945317618 \quad 2.69975431602293$

N $-0.10051944044906 \quad 1.80850143623413 \quad 3.01513074585336$

N $-1.85988373318523 \quad 0.12213526255306 \quad 2.65240189010180$

N $-0.22562375665942 \quad-1.25891615932203 \quad 1.33962527425646$

C $-2.42681671900802 \quad 1.49322491197457 \quad-0.40425132838032$

C $-1.32034302782139 \quad 2.44676685097043 \quad-0.47139832882546$

C $-1.35175919288659 \quad 3.83872897108105 \quad-0.65072491029406$

H $-2.30278576894050 \quad 4.37597324028041 \quad-0.71868330323175$

C $\quad-0.13379459749157 \quad 4.53366212371008 \quad-0.74692688178097$

C $1.08570099177626 \quad 3.83691756635811 \quad-0.69430457640982$

H $2.03723094680589 \quad 4.36896774499395 \quad-0.79481507150752$

C $1.05698315691116 \quad 2.44702877438861 \quad-0.50270048476098$

C $2.17480137128768 \quad 1.50854685587265 \quad-0.41371183434136$

C $2.80645426787524 \quad-0.78925603679976 \quad 0.07593048896114$

H $3.41896096713477-0.86073819388091 \quad-0.84099626162629$

H $2.24749483334073 \quad-1.73129852233102 \quad 0.18201466123180$

C $3.73247492203320-0.60293313227203 \quad 1.27792623171791$

H $4.44472912931316 \quad-1.45041763776206 \quad 1.28309317972613$

H $4.33293360217573 \quad 0.31642938084099 \quad 1.16748669047524$

C $3.00482655697808 \quad-0.59905982359215 \quad 2.61991143889161$

H $2.50648131509628 \quad-1.57109114650191 \quad 2.76283454458128$
} 
H 3.74008992077669

C 2.22861343307791

C 1.03508778447224

C 0.94831990315958

H 1.85174735879406

C -0.32901876906953

C -1.48872285723780

H -2.48083526149090

C -1.34509875732053

C -2.37036177354070

C -2.51689987335697

H -2.60577018116151

H -1.37882183085884

C -3.61913746206550

H -4.38683640456687

H -4.13508761621444

C -3.02850502493092

H -2.49735322972537

H -3.83188602943797

C -3.83753153429624

H -4.04367263522693

H -3.98994945384171

H -4.58420048614029

C 3.56653542616702

H 4.00220993609958

H 3.53947463639979

H 4.24901271795357

C 3.58383208690543

H 4.39345143336709

H 3.59354099213746

H 3.81066355738925

C -3.82542203257288

H -4.30778164319646

H -3.94864322694924

H -4.36820057570286

C -1.45328674878391

H -1.57330403259650

H -2.38571140355448

H -1.23806440928551

C 0.03367677903815

H -0.84939209598562

H 0.94532555604175

H 0.05476723357408

C 1.36705811669700

H 1.23064066843055

H 2.31751275936395

H 1.39950485276546

C 0.14923896260674

H 1.05954568856009

H -0.73649881731685

H 0.15797333751455

C 1.49171169047955

H 1.32925380349370

H 1.53592429327993

H 2.44405002340802

C -1.34776172104197

H -1.22796108426944

H -2.28210342069318

H -1.40100808802941

H -0.41954405372825

H -0.13593937074862
$-0.47158285538950$ 1.68624086044969 2.54124518682258 3.93315787167117 4.54784722157750 4.52476799110215 3.73377880651851 4.19602095691992 2.34222623728603 1.30976493952852 $-1.08186089807154$ $-1.80514078553104$

$-1.64911225959150$ $-1.05503905253796$ $-0.28960807907528$ $-2.03196805277036$ $-0.83699066552653$ $-1.75459448020217$ $-0.65817380714840$ 1.92294931910512 1.86094345043574

2.97345653774118

1.30284694140484 1.96524634509670 2.53739869693308

2.64816131213407

1.13322885541027

2.27420088570416

1.52867308115862

2.79314715194399

3.04122662403737

1.60302560140499

1.95968072119981

2.40262968617450

0.70832441821878

$-0.73842879892210$

0.35597723847152

$-1.18056028148953$

$-1.16831943722120$

$-2.90984729297849$

$-3.27700667723528$

$-3.19739214753953$

$-3.36440612362677$

$-0.62413547947883$

$-1.07605250542303$

$-0.98460958906942$

0.47626568361363

$-2.78283715175789$

$-3.10329136830572$

$-3.15473871733249$

$-3.20053360144031$

$-0.47173618946697$

$-0.88696338311945$

0.63104188586780

$-0.85875576032514$

$-0.53741084970218$

$-0.99408026755288$

$-0.90722778592197$

0.56171249815040

5.61276295814807

5.62147354496516
3.43477589024460 2.97728084578292 3.07281502170588 3.21702014159502 3.28433073931039 3.27518409686256 3.21085097938961 3.25100567882449 3.07204697473755 2.92160080431425 2.28210954621496 3.10948352561604 1.62827502901208 1.25701247150793 1.47509155536411 1.25977767294896 $-0.14718343376785$ $-0.44070256834386$ $-0.88155438521851$ $-0.67322021213066$ $-1.76235412059708$ $-0.37116745740225$ $-0.15304576105140$ $-0.73223467297977$ 0.11171606134377 $-1.60245139225454$ $-0.97011004397878$ 3.22413995748613

3.21380730637420 4.20309604991393 2.45676687825858 3.11679484568621 2.18392253905221 3.87054321511344 3.46983914800815 $-3.04717117557551$ $-3.14336777528992$ $-2.66540271805220$ $-4.04382209900253$

$-1.81108232274057$

$-1.25781189190960$

$-1.25669459697323$

$-2.82046830616655$ $-2.99876458273229$ $-3.99936422422705$ $-2.57527193275104$ $-3.10130388312556$ 4.51188654011175 3.97403564247918 3.96668694288891 5.53739015311425 5.64912761301662 6.66207431442037 5.71167670015910 5.25318948008393

5.60655719101280 6.60722242219135 5.15331243198196 5.71367246649799 3.37393779263558 $-0.87995770633271$

${ }^{2} \mathrm{TS}_{\mathrm{NH}}(\mathrm{S}=0)$

$\mathrm{E}=-4923.75080445 E_{\mathrm{h}}$

Co $0.09375248183909 \quad-0.41297201069313$

0.02164144006759 


\begin{tabular}{|c|c|c|c|}
\hline & -0.0 & & \\
\hline & & & \\
\hline & 21 & 99 & 161 \\
\hline & 554 & 9736 & \\
\hline & 613181 & 8169 & 6678 \\
\hline & .22476335869284 & 57074940428 & -10 \\
\hline & .73164995385315 & 18279 & \\
\hline & 2831828 & 021 & \\
\hline & 3290857922 & -1.7 & 3.67 \\
\hline & .85412149894250 & -0.0 & 2.67 \\
\hline & .50782367848307 & 9021 & -0.828 \\
\hline & .4500332735 & -2.3 & -1.4 \\
\hline & 43098 & -3.4 & -2.3 \\
\hline & .52330145272358 & 0026416 & -2.6 \\
\hline & 0.36779753794831 & 2473564510 & -2.82263 \\
\hline & -0.88 & -3.5 & -2.4 \\
\hline & -1.8 & -4. & \\
\hline & 5227137 & 081 & \\
\hline & 034815 & 6500 & -0.8 \\
\hline & -2.58 & 2085 & 0.7 \\
\hline & 9478 & & 0.2 \\
\hline & 5443 & 35 & 1. \\
\hline & -2.93860355523007 & 94470671 & \\
\hline & 88944834707 & 7299705 & 2.7 \\
\hline & 50394086428 & 98 & 1.8 \\
\hline & -1.8 & 90 & \\
\hline & -0.52 & & \\
\hline & 91727139 & 392 & 4.9 \\
\hline & -0.9 & 404 & 5.3 \\
\hline & 1.13 & 44 & \\
\hline & & & \\
\hline & 2886654570 & -2.9 & 5.3 \\
\hline & 1.73 & -1.8 & 4.0 \\
\hline & 2.5 & -0.8 & \\
\hline & & & \\
\hline & 1873267295 & 2615623 & 02 \\
\hline & 1.71 & 1.7 & 1.5 \\
\hline & 3.05 & 0.3 & .5 \\
\hline & 3.36 & & \\
\hline & 3.9 & & \\
\hline & 3.95 & 57 & -1. \\
\hline & 4.46 & 94 & 48 \\
\hline & 4.05 & & \\
\hline & & & 1156 \\
\hline & -3.4 & -2 & -1 \\
\hline & -4.23 & -1 & -0 \\
\hline & 2583234 & 73 & -0 \\
\hline & -3.6 & & \\
\hline & -3.0 & $-3 . c$ & 3.7 \\
\hline & -2.85 & -3.8 & 4.4 \\
\hline & 78946036122 & & 2.8 \\
\hline & -3.8 & 5161497 & 4. \\
\hline & 4.03 & 59 & \\
\hline & 4.4712630 & 107 & 574 \\
\hline & 35755664577 & 7924622 & 5499667 \\
\hline & 4.26 & & 4756 \\
\hline & 0.746 & 2.7 & \\
\hline & 0.66 & 3.4 & -1.9 \\
\hline & 1.8070 & & 78811970418 \\
\hline & 02173151 & 78466255 & 81032 \\
\hline & -1.55825550826294 & & 59341424923 \\
\hline & -1.48135210307432 & 01010415 & -3.1031343340411 \\
\hline & -2.18689297346624 & 1.87102390602448 & -1.4676866869421 \\
\hline & -2.01744337634419 & 0.50208083094396 & -2.6332420716713 \\
\hline & 1.08202962794898 & 0.61074596960763 & -3.06338958116560 \\
\hline & 0.66233080931653 & -0.31324566010086 & -3.499844279653 \\
\hline
\end{tabular}




$\begin{array}{llll}\text { H } & 2.13363188793293 & 0.435388877787456 & -2.77515864660380 \\ \text { H } & 1.04347352066084 & 1.42129477952337 & -3.81736297619272 \\ \text { C } & -1.66178787360662 & 0.38693139693680 & 5.47890988164272 \\ \text { H } & -1.21424991499411 & -0.52453004377955 & 5.91431448507117 \\ \text { H } & -2.63680307563877 & 0.13475395915500 & 5.02652889548903 \\ \text { H } & -1.81893742535204 & 1.13249033835549 & 6.28171555317020 \\ \text { C } & 0.90659766816057 & 1.64723668242299 & 5.16431268042096 \\ \text { H } & 0.57824803842531 & 2.30968468220339 & 5.98832734505805 \\ \text { H } & 1.59059811201926 & 2.20216473948526 & 4.49758379035713 \\ \text { H } & 1.43462093858689 & 0.77418664152291 & 5.58868471595450 \\ \text { C } & -1.36110033246103 & 2.60261380111664 & 3.64261384486545 \\ \text { H } & -1.56769236377720 & 3.26761308338344 & 4.50323333242615 \\ \text { H } & -2.31332878999564 & 2.34518863959931 & 3.14461378138534 \\ \text { H } & -0.70168723888229 & 3.11636579173231 & 2.91974907081525 \\ \text { H } & 1.40743842461849 & -4.59592300723272 & 6.12305822378665 \\ \text { H } & 0.42980487802255 & -4.85180073272201 & -3.53090822293307\end{array}$

$\left[\left({ }^{2} \mathrm{PDI}_{2}\right) \mathrm{Co}_{2}\left(\mu-\mathrm{N}_{3}\right)\left(\mathrm{PMe}_{3}\right)\right]^{3+}\left({ }^{2}<\mathrm{Co}_{2} \mathrm{~N}_{3}>^{3+}, \mathrm{S}=0\right)$

$\mathrm{E}=-4572.35758092 E_{\mathrm{h}}$

Co $0.03969934136958-0.06699264459164$

Co $0.01101188868961 \quad-0.00035990554926$

$\begin{array}{lll}\text { N } & -0.15764726243860 & 1.45119932294585\end{array}$

N $-0.80709793119665 \quad 2.47802079704193$

$\begin{array}{lll}\text { N } & -1.36919053361648 & 3.47485379627107\end{array}$

P $-0.17313635471090 \quad 0.99091058836020$

\begin{tabular}{lll}
$N$ & 1.95903555212931 & 0.01320316117651 \\
$N$ & 0.330678395822 & -1.725233501292 \\
\hline & $-C$
\end{tabular}

N $0.33068783952822 \quad-1.72523235017292$

N $-1.80360069427139-0.62565671151483$

N $-1.83405334822584 \quad-0.59804315954811$

N $0.30852623369369-1.67855327570703$

N $1.95120999840590 \quad 0.07807231808212$

C $2.55571708415442-1.05110964590572$

0.15089239578584

2.60640212985284

1.35445477818243

1.36327316364948

1.37608578614812

4.60454248989884

0.05468995318828

$-0.54295389513173$

0.02611815286926

2.64127986032004

3.24117703182509

2.66336920423673

C $1.60474152662297 \quad-2.11352807153629 \quad-0.79437238978308$

C $1.85015896641835 \quad-3.38660104127120 \quad-1.32947289213468$

H $2.87153517318364 \quad-3.72011391787862 \quad-1.53795229716845$

C $0.75708046906393 \quad-4.22943573999563 \quad-1.59678214951511$

C $-0.55396602318412 \quad-3.79457244180594 \quad-1.33731692282073$

H $-1.41331446660802 \quad-4.43918322394378 \quad-1.54756160778198$

C $-0.73831758111364 \quad-2.51148746016670 \quad-0.80411638168472$

C $-1.99641746966993 \quad-1.83374811733278 \quad-0.43997840844832$

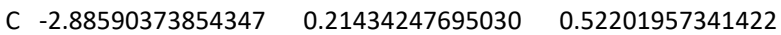

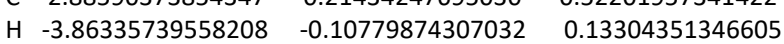

$\begin{array}{llll}\text { H } & -2.70741933293955 & 1.23557350771469 & 0.15144030916027\end{array}$

C $-2.91612343260366 \quad 0.21380701822707 \quad 2.08812288981979$

\begin{tabular}{lllll} 
H & -2.79495174844961 & 1.24159950247107 & 2.45559783668075 \\
\hline
\end{tabular}

H $-3.89377737558012 \quad-0.14234382420717 \quad 2.44372302633444$

$\begin{array}{llll}\text { C }-2.01235740305000 & -1.82668300698591 & 3.06618157818677\end{array}$

C $-0.75747877425150 \quad-2.48627624118697 \quad 3.45229216513748$

$\begin{array}{llll}\text { C }-0.55697361102959 & -3.78392768543693 & 3.94481411807755\end{array}$

H $-1.40850539090116 \quad-4.44897871319801 \quad 4.12062761696058$

C $0.75677327748281 \quad-4.21068375809750 \quad 4.20553376622362$

C $1.84289754053731 \quad-3.35138765236400 \quad 3.96419605025111$

H $2.87030038994982 \quad-3.67801050668481 \quad 4.15481640601802$

C $1.58629556127826 \quad-2.06372819025092 \quad 3.47181190573182$

C $2.53944052309332 \quad-1.00770378007195 \quad 3.10173011712584$

C $2.67604476883722 \quad 1.21975591446407 \quad 2.10848830009132$

H $3.70288829166914 \quad 1.26189072862175 \quad 2.50200599419935$

H $2.15156585280462 \quad 2.12977991789240 \quad 2.42378142516351$

C $2.70238493831842 \quad 1.16772371698513 \quad 0.54292206368186$

H $2.20789053048808 \quad 2.06752977970404 \quad 0.14558876621420$

H $3.74265491023740 \quad 1.15817863625187 \quad 0.18388811078524$

C $4.03300361326185 \quad-1.21610719302363 \quad-0.59409383487028$

H $4.37713155243922 \quad-0.63487258200729 \quad-1.47456682413943$

H $4.30017151688778 \quad-2.27208579418932 \quad-0.76361237642446$

H $4.59720964333410-0.85286531494120 \quad 0.28319193467562$ 


$\begin{array}{llll}\text { C } & -3.29239123833906 & -2.55788063704990 & -0.59730437835169 \\ \text { H } & -4.16572789710546 & -1.99493971183276 & -0.23506670719440 \\ \text { H } & -3.24400506383658 & -3.52438798464193 & -0.05678887579892 \\ \text { H } & -3.45037786030304 & -2.80346358469636 & -1.66713841384065 \\ \text { C } & -3.29977959789959 & -2.57952007204266 & 3.15627890278270 \\ \text { H } & -3.50076787856100 & -2.83510336658760 & 4.21652528283723 \\ \text { H } & -3.21104374541623 & -3.54005663028412 & 2.61163413748774 \\ \text { H } & -4.16707425965827 & -2.03092462488861 & 2.75983617536456 \\ \text { C } & 4.00652995680931 & -1.26764584074622 & 3.20143325290480 \\ \text { H } & 4.62899587620464 & -0.41735901282773 & 2.88524236946217 \\ \text { H } & 4.27043865843361 & -2.15079087238754 & 2.58568961727459 \\ \text { H } & 4.26548984520311 & -1.52396132787656 & 4.24842804333675 \\ \text { C } & -1.78432698409915 & 1.76838442370965 & 4.90772708629747 \\ \text { H } & -2.58633912442419 & 1.01036987731865 & 4.89019089730504 \\ \text { H } & -1.98513399111259 & 2.55256499646573 & 4.15606906781499 \\ \text { H } & -1.75165867980308 & 2.23850060305310 & 5.90994758971184 \\ \text { C } & 0.05415889899177 & -0.19704972750104 & 5.95474594733938 \\ \text { H } & -0.04616224902613 & 0.33537642488832 & 6.91982437259448 \\ \text { H } & 1.05901796711387 & -0.65119422247018 & 5.89360665460799 \\ \text { H } & -0.71684470920111 & -0.98577043626626 & 5.89382064482899 \\ \text { C } & 1.04005823348947 & 2.30802436698683 & 4.88947825787767 \\ \text { H } & 0.85690482819781 & 2.72990065773757 & 5.89697428059264 \\ \text { H } & 0.91776260075864 & 3.10826543954288 & 4.13811106146270 \\ \text { H } & 2.06482494752172 & 1.90076126012491 & 4.84901590877535 \\ \text { H } & 0.93529311084433 & -5.21931015684408 & 4.59438161978023 \\ \text { H } & 0.92883123930451 & -5.22855033140784 & -2.01298119180977\end{array}$

\section{$\left.<^{2} \mathrm{TS}_{\mathrm{NN}}\right\rangle(\mathrm{S}=0)$}

$\mathrm{E}=-4572.31770429 E_{\mathrm{h}}$

Co $0.04127643903794 \quad-0.02970699959065$

Co $0.00420985835827 \quad-0.00588299000353$

N $-0.00301566009321 \quad 1.30885958701354$

$\begin{array}{lll}N & -1.10212663473348 & 2.51963200402387\end{array}$

N $-1.17550534299546 \quad 3.63695680815513$

P $-0.17830949534906 \quad 1.05245705194099$

N $1.99525926373792 \quad-0.00768245505602$

N $0.32496845757514 \quad-1.66907926362783$

N $-1.80318830746765 \quad-0.59967268156753$

N $-1.84457422074420 \quad-0.63497503391176$

N $0.30197875236086 \quad-1.70287259374364$

$\begin{array}{lll}\text { N } & 1.96320496776007 & 0.03943374945075\end{array}$

C $2.56215011964262-1.06677926893788$

C $1.59076667858358-2.08363999319936$

C $1.83469115287538 \quad-3.34502036074204$

H $2.85786139007190 \quad-3.68652780678282$

$\begin{array}{lll}\text { C } & 0.73496946354079 & -4.15928043589658\end{array}$

C $-0.57351969194600 \quad-3.71045808990408$

H $-1.43722749990958-4.33965159918691$

C $-0.74706550891629-2.43846052223425$

C $-1.99769269581229-1.77456675892621$

C $-2.89152350099675 \quad 0.21385419449092$

H $-3.86229400832641 \quad-0.09623004950424$

H $-2.71228453823318 \quad 1.24852015406799$

C -2.938897481908590 .16104679962952$

H $-2.84446717901897 \quad 1.18045200262516$

H $-3.91116593389998 \quad-0.22934998738649$

C $-2.01303921048067-1.86107643785102$

C $-0.76165260312555-2.51332468438593$

C $-0.56844277411483 \quad-3.81857851835276$

H $-1.41966161747466 \quad-4.48768350557801$

C $0.74386231524330 \quad-4.25058604222823$

C $1.83273324772658-3.39390642321376$

H $2.85872305836000-3.72906701222465$

C $1.57609551593232-2.10015973460135$

C $2.53616825069102 \quad-1.05205581836282$

0.21629034705748 2.63354730824848 1.39263687693360

1.41101139785018 1.44442793280197 4.58844070086133 0.10531605987249 $-0.63348059796998$ 0.03034592257835 2.66622936950707 3.33088710946498 2.72026788317625 $-0.41205675930681$ $-0.85118081087515$ $-1.41318707983446$ $-1.59896072328200$ $-1.73450663798691$ $-1.48440875662729$ $-1.72088435958504$ $-0.92024134822345$ $-0.51656122950738$ 0.55415453798047 0.14100806808135 0.22377439104215 2.11490567361735 2.51151008729837

2.44862727297123 3.10231276220135 3.51808700544309 3.99486153490955 4.15570911547320 4.25532281963267 4.01833197456401 4.20104341614760 3.54074655425715 3.16141196129746 


$\begin{array}{llll}\text { C } & 2.70752781683116 & 1.17473641403631 & 2.17800558223495 \\ \text { H } & 3.72869322991381 & 1.20836960510123 & 2.58646751813618 \\ \text { H } & 2.18703972878695 & 2.08836754886030 & 2.48614565332985 \\ \text { C } & 2.75570977301045 & 1.12670343878480 & 0.61547838031738 \\ \text { H } & 2.28746826449980 & 2.03712018948400 & 0.21245172744472 \\ \text { H } & 3.79982799881329 & 1.09669056325465 & 0.27230545548910 \\ \text { C } & 4.02439879085842 & -1.33141675695537 & -0.56864715498149 \\ \text { H } & 4.27944991113197 & -1.37600036860321 & -1.64714819694577 \\ \text { H } & 4.26330422513644 & -2.32582481079208 & -0.14404433239395 \\ \text { H } & 4.66950251967193 & -0.58225773367678 & -0.08650077457074 \\ \text { C } & -3.29785636897489 & -2.48377996099754 & -0.70928923881241 \\ \text { H } & -4.17156497854266 & -1.92788372299027 & -0.33846785464604 \\ \text { H } & -3.25951041589681 & -3.46248546496434 & -0.19082741345016 \\ \text { H } & -3.44590526749277 & -2.70050444923539 & -1.78602153929290 \\ \text { C } & -3.28891639404299 & -2.63860774488584 & 3.15309261777659 \\ \text { H } & -3.48580506248704 & -2.95841116159085 & 4.19537440278943 \\ \text { H } & -3.17915806054741 & -3.56326094427672 & 2.55300016630486 \\ \text { H } & -4.16673716028935 & -2.08782257189797 & 2.78477259730058 \\ \text { C } & 4.00216793858819 & -1.32761495054573 & 3.24346280266329 \\ \text { H } & 4.62944291446828 & -0.49282793028918 & 2.89738358041506 \\ \text { H } & 4.24272656603350 & -2.22391283030593 & 2.63711723071666 \\ \text { H } & 4.27823937592513 & -1.56962432773016 & 4.28899125514766 \\ \text { C } & -1.85293123619923 & 1.62227165772553 & 4.99553629655363 \\ \text { H } & -2.55788912211721 & 0.77293178283679 & 5.00124479471567 \\ \text { H } & -2.18746705972349 & 2.38893625134810 & 4.27474661306763 \\ \text { H } & -1.82085190066351 & 2.07400934148927 & 6.00605005800239 \\ \text { C } & 0.30235727075429 & -0.02561808538683 & 5.96646984752157 \\ \text { H } & 0.24288747272142 & 0.55200856703492 & 6.90883230110435 \\ \text { H } & 1.33816750473572 & -0.38097021985960 & 5.82541185519771 \\ \text { H } & -0.38139303816050 & -0.89108472532620 & 6.02541870336454 \\ \text { C } & 0.86407556942365 & 2.52890487011872 & 4.73321420086835 \\ \text { H } & 0.63120972874193 & 3.01999233097436 & 5.69845954421820 \\ \text { H } & 0.64989851212491 & 3.23302473006929 & 3.90925849558192 \\ \text { H } & 1.92988274271296 & 2.24556368448175 & 4.72193303200285 \\ \text { H } & 0.91945603089710 & -5.26336457951936 & 4.63382194399791 \\ \text { H } & 0.89842315340565 & -5.14868692016823 & -2.17559993124030\end{array}$

\begin{tabular}{|c|c|c|c|}
\hline \multicolumn{4}{|c|}{$\left[\left({ }^{2} \mathrm{PDI}_{2}\right) \mathrm{Co}_{2}(\mu-\mathrm{N})\left(\mathrm{PMe}_{3}\right)\right]^{3+}\left({ }^{2}<\mathrm{Co}_{2} \mathrm{~N}>^{3+}, \mathrm{S}=0\right)$} \\
\hline & 4773103807 & 49221481 & . \\
\hline & .07482752987737 & 05958273 & \\
\hline & -0.13304392681180 & 411 & \\
\hline & 0.06866466358997 & 1.18023 & -1.5 \\
\hline & $2.12179964 \varepsilon$ & -0.6 & \\
\hline & 0.24 & & \\
\hline & -1.7 & & \\
\hline & & & \\
\hline & & & \\
\hline & 1.96076921 & -0.2 & \\
\hline & 2.5340209 & & \\
\hline & 1.46 & & \\
\hline & 1.55 & & \\
\hline & 45 & & \\
\hline & 8 & -3 & \\
\hline & & & \\
\hline & & & \\
\hline & & & \\
\hline & & & \\
\hline C & & & \\
\hline & & & \\
\hline & -2.3 & & \\
\hline & .90858559606132 & 9005 & 1.8940 \\
\hline & 3.65283620661337 & -0.72838858128131 & 2.5507315 \\
\hline &  & 60389620 & 1.582 \\
\hline & & $400<40$ & \\
\hline
\end{tabular}




\begin{tabular}{|c|c|c|c|}
\hline & & & \\
\hline & 383 & -3.37060860318522 & \\
\hline & -1.06449732856088 & -4.00852051596909 & \\
\hline & 1.01878837279460 & -3.39859324935185 & 6.054 \\
\hline & 862 & -2.587 & 5.51285 \\
\hline & 3.04708699448592 & -2.607 & 647495 \\
\hline & 1.7 & -1.7 & \\
\hline & & -0.8 & \\
\hline & & & \\
\hline & & & \\
\hline & & & \\
\hline & & & \\
\hline & & & \\
\hline & & & \\
\hline & & & \\
\hline & & & \\
\hline & & & \\
\hline & & & \\
\hline & 356 & & -1 \\
\hline & & & \\
\hline & & & \\
\hline & & & \\
\hline & & & \\
\hline & & -3 & \\
\hline & & -3 & \\
\hline & & -2 & \\
\hline & & -0.6 & \\
\hline & & & \\
\hline & & -1. & \\
\hline & & & \\
\hline & & & \\
\hline & & & \\
\hline & & & \\
\hline & 0.70 & 2.9 & -0.0 \\
\hline & -1.610 & 1206 & -1.8 \\
\hline & 39933357 & 7589 & 67297 \\
\hline & 3564 & 0573 & 5272 \\
\hline & -2.2590 & 774 & 55724 \\
\hline & 0.66 & 0.65 & 3700 \\
\hline & & -0. & 6108 \\
\hline & & & 2472 \\
\hline & 0.6 & 1.5 & 2322 \\
\hline & 1.2 & -4.0 & \\
\hline & 0.42548155484150 & -4.58218923965042 & -4.1048697497644 \\
\hline
\end{tabular}

$\left[\left({ }^{3} \mathrm{PDI}_{2}\right) \mathrm{Co}_{2}\left(\mu-\mathrm{N}_{3}\right)\left(\mathrm{PMe}_{3}\right)\right]^{3+}\left({ }^{3}<\mathrm{Co}_{2} \mathrm{~N}_{3}>^{3+}, \mathrm{S}=0\right)$

$\mathrm{E}=-4650.83533397 E_{\mathrm{h}}$

Co $-0.00775919964502 \quad-0.07214510190756-0.05638190948280$

Co $-0.01272192386784 \quad-0.03723054588064 \quad 2.43238458511592$

P $0.03466261602291 \quad-0.88479564757965 \quad-2.14677626575083$

$\begin{array}{llll}\text { N } & -1.94513421407751 & 0.06802047518829 & -0.16117694491804\end{array}$

$\begin{array}{llll}N & -0.10206863751336 & 1.68685933516327 & -0.51195906933662\end{array}$

$\begin{array}{llll}\text { N } & 1.90201029969679 & 0.26986599743531 & -0.16282056265666\end{array}$

N $1.88105828880243 \quad 0.26271374196719 \quad 2.67349228395412$

N $-0.09217794656699 \quad 1.71331745163156 \quad 2.91587159480753$

N $-1.92471063559626 \quad 0.09285184672859 \quad 2.66567768614200$

N $-0.00737759293791 \quad-1.54759410151664 \quad 1.19433199185994$

N $0.46945379591485 \quad-2.66366666759621 \quad 1.20560053038136$

N $0.87082549465613 \quad-3.73666421129689 \quad 1.21800956445274$

C $-2.39368954596375 \quad 1.25638483256476 \quad-0.47961835406037$

C $-1.32662838927158 \quad 2.25435805682276 \quad-0.62835285530134$

C $-1.42613495923894 \quad 3.63532799889129 \quad-0.85134060688569$

H $-2.40673331617142 \quad 4.11456970745470 \quad-0.93650349553723$

C $-0.24470211008885 \quad 4.39027950985657 \quad-0.95219714307577$

$\begin{array}{llll}\text { C } 1.00987524934904 & 3.76479953912227 & -0.84883433485687\end{array}$ 
H 1.93496967549629

C 1.05501017016295

C 2.22129409120780

C 2.91866490401326

H 3.62790967580047

H 2.40079562307838

C 3.71143324974063

H 4.41431024056818

H 4.33043346903924

C 2.88044097753817

H 2.34366965748643

H 3.55288824234219

C 2.21726010873099

C 1.06501801092403

C 1.03439099989682

H 1.96446867597566

C -0.21507490639749

C -1.40178091344587

H -2.38002615534708

C -1.30758526132353

C -2.37251386627097

C -2.80760022285113

H -3.47208724044013

H -2.16540369312654

C -3.64633784674869

H -4.35011595635203

H -4.26196323224316

C -2.83608119326616

H -2.20185950299191

H -3.53168722514862

C -3.82210904037633

H -4.47842217244617

H -3.90238234484731

H -4.20439917106337

C 3.59722117470572

H 3.87654567576541

H 3.61459507587254

H 4.36575390782455

C 3.58292526379236

H 4.33525384744753

H 3.54220800366707

H 3.92648954538030

C -3.77807672171620

H -4.18289827096590

H -3.78781204656090

H -4.45489216786538

C -1.42934417966629

H -1.57047562572310

H -2.32970393776611

H -1.26044303185527

C 0.15769942363092

H -0.69736111700820

H 1.10250805550884

H 0.14556942274774

C 1.41776835308824

H 1.28982313603817

H 2.38135887339769

H 1.40609920918553

H -0.26400478945881

H -0.30198218028418
4.34418032869596 2.38106841320751

1.50218522173307

$-0.78597306753712$

$-0.73399657080270$

$-1.75317220816564$

$-0.72331422915746$

$-1.57767031467825$

0.19153230981417

$-0.80961335124163$

$-1.77014762892631$

$-0.77262767574953$

1.48673976613133

2.39939938503532

3.78534923018176

4.35352193107437

4.42601478945087

3.67690878377966

4.15987012459915

2.29367953129421

1.27972510853281

$-1.07815686005243$

$-1.10737123277154$

$-1.97271602087896$

$-1.08353759169992$

$-0.23170851525977$

$-2.00297204558596$

$-1.09343139105518$

$-1.99157165026105$

$-1.14231892218583$

1.63673835099184

0.76539725699499

2.30002676578762

2.20966399140233

2.04011166157540

2.72754187646563

2.63865701887102

1.25573291114129

1.99543860770789

1.19972073016298

2.57649213450155

2.69695402901880

1.65655461115061

2.34557124817075

2.21527824224344

0.79306391515467

$-0.46237052129660$

0.63320655011351

$-0.95243265708515$

$-0.81464284322539$

$-2.69447249916116$

$-3.13814004743517$

$-3.01558253820538$

$-3.03722965895123$

$-0.26500179544130$

$-0.63473425737358$

$-0.62686448676398$

0.83974142879213

5.51056381742854

5.47190829269406
$-0.93282802584279$

$-0.62521025443361$

$-0.47423266023517$

$-0.01265159727753$

$-0.85843050958525$

$-0.09100508153321$

1.29803436965080

1.29130276721274

1.31630019080394

2.58225454921241

2.63460146877482

3.45851758508678

2.99947456199950

3.05964242248863

3.26971137121985

3.37324576544886

3.34625563303686

3.25775737684561

3.35029670674019

3.04977777168601

2.99126382015619

2.58557618692370

3.46815803966875

2.62127330027113

1.30383414150510

1.30676590415619

1.30759842166063

0.00293967512185

$-0.02810249454746$

$-0.85435855533160$

$-0.70093161377600$

$-0.85299889709391$

$-1.58270948182463$

0.16811649618721

$-0.69774189626764$

0.12642592402053

$-1.62902289518002$

$-0.76856899611544$

3.32907057092778

3.43798816769318

4.27119272151113

2.54118554718976

3.33035535535870

2.56080154708580

4.28706169551663

3.41794190889838

$-3.13411128644274$

$-3.14604988770869$

$-2.72980379918856$

$-4.16955164029890$

$-2.17071756878446$

$-1.63057165585608$

$-1.69812879614109$

$-3.22373439420228$

$-3.14655556741648$

$-4.18183092298552$

$-2.75291987163971$

$-3.15488064213993$

3.49574644395235

$-1.11760611101464$

$<^{3} \mathrm{TS}_{\mathrm{NN}}>(\mathrm{S}=0)$

$E=-4650.79493130 E_{h}$

Co $-0.00906764710219 \quad-0.07485888012608 \quad-0.04687721549551$

Co $-0.01072565024192 \quad-0.06017658497208 \quad 2.39093306639866$ 


\begin{tabular}{|c|c|c|c|}
\hline ? & 0.02959081384347 & -0.96435886381148 & -2.10547636225763 \\
\hline & -1.96605488239485 & 0.09412520664609 & -0.16776472354364 \\
\hline & -0.10925635557271 & 1.70162338792178 & -0.57708082752541 \\
\hline & 1.91038790240528 & 0.29716241770345 & -0.18069046732256 \\
\hline N & 1.89482822710496 & 0.26118445655495 & 2.67856907212206 \\
\hline  & -0.08777432401247 & 1.70199956303062 & 2.97976672110043 \\
\hline$\sqrt{ }$ & -1.92733041447554 & 0.10520354833041 & 2.65728356904490 \\
\hline$\sqrt{ }$ & -0.13753441076493 & -1.38566191508965 & 1.19727825734560 \\
\hline$N$ & 0.79345570631988 & -2.72643834063486 & 1.23040302771242 \\
\hline$\sqrt{ }$ & 0.71818574352955 & -3.84188586408940 & 1.31039332574105 \\
\hline$C$ & -2.40201378294869 & 1.28144834156892 & -0.50186319155342 \\
\hline- & -1.33157089042172 & 2.27563040937524 & -0.65662259988681 \\
\hline$C$ & -1.43601027190256 & 3.66096205863216 & -0.85173568878149 \\
\hline $\mathrm{H}$ & -2.41661503036502 & 4.14320874129305 & -0.91795781122496 \\
\hline C & -0.25551386156676 & 4.41751011639843 & -0.95285367844317 \\
\hline C & 0.99989604571075 & 3.79018788238548 & -0.86710353401334 \\
\hline $\mathrm{H}$ & 1.92392577155670 & 4.37168516526710 & -0.94193005969642 \\
\hline$C$ & 1.04305373822099 & 2.40188330781696 & -0.67205493212845 \\
\hline C & 2.21280724131207 & 1.52774925362195 & -0.51555780186352 \\
\hline C & 2.94741300902882 & -0.73852465949224 & -0.03307809694196 \\
\hline $\mathrm{H}$ & 3.65626901875515 & -0.67032110014900 & -0.87803811481889 \\
\hline $\mathrm{H}$ & 2.44567443036883 & -1.71351022243426 & -0.10987441824088 \\
\hline C & 3.73851960031995 & -0.66456818562955 & 1.27712144783454 \\
\hline $\mathrm{H}$ & 4.46962642773899 & -1.49550708701047 & 1.26196362522882 \\
\hline $\mathrm{H}$ & 4.32392055350869 & 0.27242110614855 & 1.30468560887519 \\
\hline$C$ & 2.91239983417118 & -0.78880296397950 & 2.56102301210050 \\
\hline $\mathrm{H}$ & 2.39503456123344 & -1.75977783364911 & 2.59566937190350 \\
\hline $\mathrm{H}$ & 3.58551866278360 & -0.75547250469223 & 3.43702029397889 \\
\hline C & 2.22004899142074 & 1.48164428054299 & 3.03047060930823 \\
\hline C & 1.06840768049746 & 2.38919659890507 & 3.10963570156265 \\
\hline C & 1.04489595612260 & 3.77879517000646 & 3.29459571207316 \\
\hline $\mathrm{H}$ & 1.97639852894448 & 4.34478309653168 & 3.39412123259841 \\
\hline C & -0.20178832534454 & 4.42781031809719 & 3.34747479745427 \\
\hline C & -1.39101410395044 & 3.68411436974110 & 3.25132759077729 \\
\hline $\mathrm{H}$ & -2.36702907864771 & 4.17565917333164 & 3.31984639641872 \\
\hline C & -1.29931171770108 & 2.29655242744006 & 3.06991979844247 \\
\hline C & -2.36676324839457 & 1.29155913343036 & 2.99622961624610 \\
\hline C & -2.81992517009213 & -1.05982108288460 & 2.57690159711382 \\
\hline $\mathrm{H}$ & -3.47815591339742 & -1.08745831325092 & 3.46446732406012 \\
\hline $\mathrm{H}$ & -2.18385839665958 & -1.95862988011680 & 2.60275879385706 \\
\hline C & -3.66684357600931 & -1.04971114398956 & 1.30163896879697 \\
\hline $\mathrm{H}$ & -4.36508377529251 & -0.19189859117279 & 1.31351518927602 \\
\hline $\mathrm{H}$ & -4.28958679930909 & -1.96469154872221 & 1.30568348260694 \\
\hline C & -2.86554253382413 & -1.06091812123373 & -0.00416318644025 \\
\hline $\mathrm{H}$ & -2.23525104761415 & -1.96231582266457 & -0.03811929650629 \\
\hline $\mathrm{H}$ & -3.56707785452976 & -1.10482863393894 & -0.85698423148019 \\
\hline C & -3.82607152465157 & 1.67218355856030 & -0.73850165226099 \\
\hline $\mathrm{H}$ & -3.89842177343669 & 2.27175332525490 & -1.66623673650576 \\
\hline $\mathrm{H}$ & -4.18658263265421 & 2.31820330641080 & 0.08839719042987 \\
\hline $\mathrm{H}$ & -4.50510357668135 & 0.80927569408691 & -0.81896670852473 \\
\hline C & 3.58301642933416 & 2.07730019044314 & -0.75067646419655 \\
\hline $\mathrm{H}$ & 3.84850046918496 & 2.79152471226440 & 0.05503250239412 \\
\hline $\mathrm{H}$ & 3.59345891921833 & 2.64988403292763 & -1.69852355679614 \\
\hline $\mathrm{H}$ & 4.36548797362348 & 1.30466595489505 & -0.79708426865314 \\
\hline C & 3.58672730286031 & 1.99396880187306 & 3.35367588382863 \\
\hline $\mathrm{H}$ & 4.34502443776223 & 1.20236060010456 & 3.45325218100922 \\
\hline $\mathrm{H}$ & 3.54961989512935 & 2.57003949349785 & 4.29880898576296 \\
\hline $\mathrm{H}$ & 3.92143787076008 & 2.69917310678864 & 2.56520414369768 \\
\hline C & -3.77405868945712 & 1.66828582822436 & 3.33248011387243 \\
\hline $\mathrm{H}$ & -4.18598498714873 & 2.33334057596117 & 2.54524908083196 \\
\hline $\mathrm{H}$ & -3.78606508436109 & 2.24808691572279 & 4.27595522247666 \\
\hline $\mathrm{H}$ & -4.44625783942423 & 0.80322676550384 & 3.43945248105421 \\
\hline C & -1.43207893941692 & -0.56128288063950 & -3.10584909997582 \\
\hline $\mathrm{H}$ & -1.56758497058474 & 0.53421287330355 & -3.14966067845366 \\
\hline $\mathrm{H}$ & -2.33548200913230 & -1.03483897037011 & -2.69050633230505 \\
\hline $\mathrm{H}$ & -1.26358354079387 & -0.94477256105939 & -4.13008331812360 \\
\hline
\end{tabular}




$\begin{array}{llll}\text { C } & 0.14751962665093 & -2.77433802558370 & -2.09536251659841 \\ \text { H } & -0.68925578190247 & -3.20644633542165 & -1.51817336776062 \\ \text { H } & 1.10736505305519 & -3.08608862934299 & -1.64848170045131 \\ \text { H } & 0.10039638480252 & -3.13860428212539 & -3.14006270619909 \\ \text { C } & 1.40939105742897 & -0.37419028450093 & -3.12932924955503 \\ \text { H } & 1.27578798192077 & -0.77560266919892 & -4.15187283962322 \\ \text { H } & 2.37494755999354 & -0.72500454047885 & -2.73171373349458 \\ \text { H } & 1.39818446819762 & 0.73013403853682 & -3.17188676092703 \\ \text { H } & -0.24665112241013 & 5.51488369549060 & 3.47967830820919 \\ \text { H } & -0.31433334022880 & 5.50195932188341 & -1.09891337497617\end{array}$

$\left[\left({ }^{3} \mathrm{PDI}_{2}\right) \mathrm{Co}_{2}(\mu-\mathrm{N})\left(\mathrm{PMe}_{3}\right)\right]^{3+}\left({ }^{3}<\mathrm{Co}_{2} \mathrm{~N}>^{3+}, \mathrm{S}=0\right)$

$\mathrm{E}=-4541.34099641 E_{\mathrm{h}}$

Co $-0.01235630973879 \quad-0.01545303793254 \quad-0.03150580747446$

$\begin{array}{llll}\text { Co }-0.01422619276092 & 0.07699223702741 & 2.43077569521477\end{array}$

$\begin{array}{llll}\text { P } & 0.03621809355229 & -1.05066994926474 & -2.01399167903959\end{array}$

$\begin{array}{llll}\text { N } & -1.97246036984212 & 0.16918954598626 & -0.14653128135068\end{array}$

$\begin{array}{llll}\mathrm{N} & -0.10759005926193 & 1.74271886462618 & -0.70683467610457\end{array}$

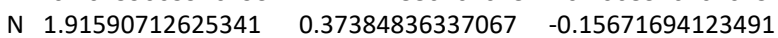

$\begin{array}{llll}N & 1.86877059200997 & 0.42348737646747 & 2.72427355638855\end{array}$

$\begin{array}{llll}\text { N } & -0.09899577824160 & 1.84280414469301 & 3.09066848831529\end{array}$

$\begin{array}{llll}\text { N } & -1.92059794676180 & 0.23920019731651 & 2.73373791707935\end{array}$

$\begin{array}{llll}\mathrm{N} & 0.04863303042791 & -1.12660892643667 & 1.25909265439382\end{array}$

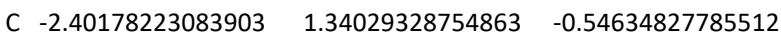



C $-1.43314640121652 \quad 3.71148495818459 \quad-0.92694007297748$

H $\quad-2.41338806672277 \quad 4.19712606232886 \quad-0.97408549783966$

C $-0.25165445537672 \quad 4.46870100561996 \quad-1.01834316929479$

C $1.00306035705223 \quad 3.84002085813241 \quad-0.93117944702827$

H $1.92731649480398 \quad 4.42490740747847 \quad-0.98188784130025$

$\begin{array}{llll}\text { C } 1.04542365460245 & 2.44829694655708 & -0.75851931230409\end{array}$

$\begin{array}{llll}\text { C } 2.21703410428416 & 1.58394103044712 & -0.55742465540081\end{array}$

$\begin{array}{llll}\text { C } 2.93334891438620 & -0.67493587224119 & 0.04456984622384\end{array}$

H $3.64799871728051-0.65905077998400 \quad-0.79772035135046$

H $2.40594584104352 \quad-1.64105643596309 \quad 0.02074256957161$

C $3.71113796611420 \quad-0.55311033730310 \quad 1.35733449890023$

H $4.44097020657740-1.38489106208819 \quad 1.37568684922005$

$\begin{array}{llll}\text { H } & 4.29688642617212 & 0.38338509689202 & 1.36609106698650\end{array}$

$\begin{array}{llll}\text { C } 2.86255788769726 & -0.65782381816162 & 2.62669866845755\end{array}$

H $2.31384001629643 \quad-1.61261858785301 \quad 2.64540446477419$

H $3.51703637533149 \quad-0.63299082817789 \quad 3.51723604943284$

$\begin{array}{llll}\text { C } 2.20517565445539 & 1.64931650493551 & 3.05225939657746\end{array}$

C $1.05441380524785 \quad 2.55040975340949 \quad 3.13543691757703$

C $1.02022298097441 \quad 3.94835305388949 \quad 3.24157059607198$

H $1.94837796360574 \quad 4.52812496446384 \quad 3.28201244835820$

C $-0.23171220860647 \quad 4.58742447410009 \quad 3.28788901731643$

$\begin{array}{llll}\text { C } & -1.41611398414637 & 3.82970801842656 & 3.24924126460423\end{array}$

H $-2.39525142294691 \quad 4.31795137965031 \quad 3.29664267946186$

C $-1.31551593353064 \quad 2.43506828180517 \quad 3.14288542625703$

C $-2.37325546755245 \quad 1.42581521375695 \quad 3.06608125571296$

C $-2.80267477162699 \quad-0.93572595640786 \quad 2.64603765883556$

$\begin{array}{llll}\text { H } & -3.45308930568287 & -0.97050065475925 & 3.53891944340757\end{array}$

H $-2.16034245087623 \quad-1.83006261638205 \quad 2.66839586803667$

$\begin{array}{llll}\text { C } & -3.66013545901369 & -0.92798487887840 & 1.37832021083038\end{array}$

H $-4.34565915180521 \quad-0.06145827958898 \quad 1.38141311628964$

H $-4.29654176120776 \quad-1.83316450526206 \quad 1.40402493517637$

C $-2.87373111273192 \quad-0.97866658602303 \quad 0.06605394512631$

$\begin{array}{llll}\text { H } & -2.24733493136355 & -1.88363832390966 & 0.05314175471872\end{array}$

H $-3.58447261047751 \quad-1.04871727333748$ - 0.77692436116381

C $-3.82807623822672 \quad 1.72568570962077 \quad-0.78090260922109$

H $-3.91300962747123 \quad 2.28417128457244 \quad-1.73244353024415$

H $-4.17121357484375 \quad 2.40941536533990 \quad 0.02259547512524$

H $-4.512073177671330 .86339625206733 \quad-0.81132179073095$

C $3.59252933518283 \quad 2.11834573144646 \quad-0.80167743894805$

H $3.86338476628157 \quad 2.84143217945750 \quad-0.00504099939008$ 


$\begin{array}{llll}\text { H } & 3.61369397079770 & 2.67513359208670 & -1.75792844740651 \\ \text { H } & 4.36526541744728 & 1.33472430579686 & -0.82907310519230 \\ \text { C } & 3.58448533986470 & 2.14865502224114 & 3.34287496821009 \\ \text { H } & 4.30735672714863 & 1.34107022727686 & 3.53811682230748 \\ \text { H } & 3.55958113460847 & 2.82179961899941 & 4.22046649179972 \\ \text { H } & 3.95991320885719 & 2.74868637310999 & 2.48833627766184 \\ \text { C } & -3.79300415755182 & 1.78694371078708 & 3.36670255051123 \\ \text { H } & -4.22030509531945 & 2.37400146559331 & 2.52763064290053 \\ \text { H } & -3.83034475450515 & 2.43570063811705 & 4.26237835223630 \\ \text { H } & -4.43829767599141 & 0.91111849803677 & 3.53681814957402 \\ \text { C } & -1.41329347779937 & -0.70673426278978 & -3.04855291810383 \\ \text { H } & -1.52434618078809 & 0.38379879097976 & -3.18520036449516 \\ \text { H } & -2.32767984215319 & -1.12634808553241 & -2.60013473848481 \\ \text { H } & -1.24557802483690 & -1.17944189140367 & -4.03543065178563 \\ \text { C } & 0.12277116280382 & -2.84695684665444 & -1.79745597249780 \\ \text { H } & -0.75661265572236 & -3.19594921646338 & -1.22868540496942 \\ \text { H } & 1.04173684698184 & -3.11247995905308 & -1.24604654612619 \\ \text { H } & 0.13529210161445 & -3.32443950734757 & -2.79693654496719 \\ \text { C } & 1.43870736882707 & -0.57133295412021 & -3.05996704993205 \\ \text { H } & 1.30565926551134 & -1.05638174596281 & -4.04623815127885 \\ \text { H } & 2.39281145015969 & -0.90565256823508 & -2.62244827184228 \\ \text { H } & 1.44607358573290 & 0.52508054588906 & -3.19512854087281 \\ \text { H } & -0.28474434135307 & 5.67971791483235 & 3.35944646057642 \\ \text { H } & -0.30914180359663 & 5.55576510108428 & -1.14393938546265\end{array}$


H $8.14604898514419 \quad 14.72317175469135$

H $9.13610007311940 \quad 13.24395929321336$

H $7.49919835760722 \quad 13.15025749225327$

C $7.45399979166928 \quad 12.82922359169197$

H $6.71653204399925 \quad 12.21527528063626$

H $8.45313137603146 \quad 12.38978966944132$

H $7.45488227515418 \quad 13.84148107951476$

C $0.43033642731405 \quad 12.11822135886807$

H $0.11828903625752 \quad 13.10277113149255$

H $-0.48843746750450 \quad 11.50814558308294$

H $1.09910869427953 \quad 11.63621823147864$

C $1.06200504203913 \quad 12.93024652218136$

H $1.88617984631279 \quad 12.70070963221997$

H $0.21651885651746 \quad 12.24171215650990$

H $0.69889922003517 \quad 13.95667427992475$

C $4.36342360323844 \quad 16.53511683205019$

H $4.48697968840738 \quad 17.62666988590767$

H $3.89041894893485 \quad 16.34237676915819$

H $3.71634701679514 \quad 16.13327606194956$

C $7.01048790517516 \quad 16.54709880396772$

H $7.13131577775640 \quad 17.61331447200440$

H $8.00682893321841 \quad 16.07014494786109$

H $6.52574253405933 \quad 16.48007792191141$

C $6.73864233979435 \quad 16.24021783783845$

H $6.07583575987680 \quad 15.97830921015053$

H $7.71928241035041 \quad 15.74687220461909$

H $6.88603974369031 \quad 17.33703420974470$

C $4.61566954625921 \quad 13.06846923303135$

H $11.37891718845277 \quad 12.30420970388981$

H $-2.68411706134953 \quad 13.96312620476063$

H $4.01908120398526 \quad 13.65231636628300$

H $5.37843020931740 \quad 12.52322869267032$

H $3.31054056413730 \quad 11.36114204664228$

H $4.33119876455113 \quad 11.34138573090608$

H 4.1039556677043914 .14193999673427

H $5.14559776974736 \quad 14.04730530834635$

H $5.79239335431942 \quad 14.84611576828022$

H 4.5155258300565014 .65436764532959

cis- $\left[\left({ }^{3} \mathrm{PDI}_{2}\right) \mathrm{Co}_{2}\left(\mu-\mathrm{N}_{3} \mathrm{PMe}_{3}\right)\left(\mathrm{PMe}_{3}\right)\right]^{3+}\left(\operatorname{cis}-{ }^{3}<\mathrm{Co}_{2} \mathrm{~N}_{3} \mathrm{P}>^{3+}, \mathrm{S}=0\right)$

$\mathrm{E}=-5111.70433713 E_{\mathrm{h}}$

Co -0.14683837471559

Co -0.23400905962854

P -0.07425229343504

N -2.06091003209992

N -0.11433124376662

N 1.78458645726206

N 1.67097232821589

N -0.23116431618777

N -2.14883743206333

C -2.42068562449185

C -1.29391295390328

C -1.30446589182888

H -2.24958124550741

C -0.07640425790969

C 1.13318309171837

H 2.09300043838357

C 1.08443178787946

C 2.73706087799088

H 3.45904890285064

H 2.15963679663476

C 3.51039895932147

H 4.19608626049897

H 4.14052863567741
12.50607836123583 12.34055973458244 13.05578119575273 4.87593486856808 4.33073552829869 4.72020331404554 4.41904084593404 5.61135633116844 5.20507901243722

5.71858166368361 4.88156267114841 12.93534812299945 13.62793548453960 13.13739473533293 13.14690343549812 8.52542129031811 8.38564514445068 9.50517955426226 7.72498813588689 9.72300582207584 9.45202004112200 9.76418081156942 10.71167101744228 6.88376393085267 6.04185581453048 6.75332247867772 6.89747111176553 12.50282555151142

8.35701897751502 9.40732043208506

13.23027322710650 13.08954877452841 12.66208034107413 11.20869403986731 6.47619750526413 5.04001228866194 12.25073020504878 11.03142003576745
$-0.09270467564217$ $-0.27799203585612$ $-0.84440730741393$ 0.19690886086294 1.72253148892031 0.15601650027482 $-0.08964654365013$ 1.43442553990275 $-0.01792114380176$ 1.43586957116520 2.37512345979107 3.77241029516789 4.31759546637992 4.45705406256325 3.74769340265113 4.27434053590555 2.35082156834149 1.38875429184852 $-0.96350369816657$ $-0.90088501874110$ $-1.89207903772768$ $-1.01616965638413$

$-1.88315048560309$ $-0.11597957575807$
$-0.18374386472037$

2.28491549345960

$-2.29811378935954$

$-0.37599156787077$

$-0.50691860286538$

$-0.22453758811951$

2.59363731861625

2.96743930947119

2.44020649267581

$-0.61466322737350$

$-0.63280010312777$

$-0.76070623963266$

$-0.85111014467783$

$-0.76154262159074$

$-0.65910162191491$

$-0.67132665409104$

$-0.53374698980635$

$-0.42717891519849$

$-0.13722529228118$

$-0.97137402964335$

$-0.26146996719446$

1.18325433407006

1.13030423286894

1.29469821637795 
C $2.62493308462926-1.19492596641728$

H $2.04047188366832-2.12118784629553$

H $3.25328105234378-1.29874916592915$

C $2.06014057489228 \quad 1.07710765498464$

C $0.95994227377084 \quad 2.04157677758619$

C $1.00529531291715 \quad 3.40985813625950$

H $1.96335130509402 \quad 3.91419335909184$

C $-0.20593212529947 \quad 4.11956907174726$

C $-1.43146675467876 \quad 3.45648526531819$

H $-2.38042580886060 \quad 3.99659728905053$

C $-1.41242206586547 \quad 2.08681294374086$

C $-2.53343224172515 \quad 1.16374786340374$

C $-3.11065640702112-1.10247670079114$

H $-3.79599298539612 \quad-1.19638609214779$

H $-2.52767326512234-2.03293998348993$

C $-3.90569207202007 \quad-0.92592156692882$

H $-4.53021594435147 \quad-0.01680769708991$

H $-4.59888681155772 \quad-1.78387831019319$

C $-3.03843727396834 \quad-0.90298655348224$

H $-2.46929252365628 \quad-1.84253052321573$

H $-3.69594155764184-0.83800088605346$

C -3.80846079626490

H -3.79574309317536

H -4.19096627171015

H -4.52083711380820

C 3.60231086307367

H 3.93680973440981

H 3.67235558143734

H 4.30530402371614

C 3.44713380384255

H 4.14003062640063

H 3.41739190487102

H 3.86523191862984

C -3.93474718529726

H -4.27217703276673

H -3.96686232241397

H -4.65526978628274

C -1.44420406315206

H -1.49387636887013

H -2.40466913680976

H -1.25251857688365

C -0.09464234478966

H -1.02146565171156

H 0.76867876621635

H -0.04588722157094

C 1.38775485316055

H 1.26823774110588

H 2.30758248483019

H 1.46512468827740

H -0.19502070739031

H -0.06149571508593

H 0.95715014571763

H -1.94107665201252

N -0.00960632104370

C 1.02043388556937

C -1.84335813010305

P -0.24397551365291

H -2.66744524339876

H 2.01632694310727

H 0.85518005769815

H -1.88925904807637

C -0.13879530457006

H 0.90628339702408

H -0.76400661423834

H -0.56664170641401

N -0.08638697568922
1.92379224580861

2.60941652989434

2.50819694115285

1.11051059702723

1.84804332534437

2.38756122757255

2.56492023862393

1.02146720487948

1.46952958048681

0.61699242119225

1.98456655575547

2.19590131139350

1.60476358472547

2.32186169579706

2.14518293155643

0.77296953945499

$-0.24382937627518$

0.85891982102235

$-0.67338186360547$

$-0.54723192832580$

$-2.65117984695181$

$-3.05640689277677$

$-3.08571856708642$

$-2.91352914995568$

$-0.28399867871496$

$-0.58267933142653$

$-0.74086964693712$

0.81697289812000

5.19199192718531

5.54919944722675

$-5.78852216261935$

$-5.40620405626875$

$-3.74708967710349$

$-4.81164467698386$

$-4.46215163459380$

$-3.72023620619164$

$-3.78132034063445$

$-4.36534041050108$

$-4.94542296163933$

$-4.66871701518466$

$-2.19973540647492$

$-1.87805822995878$

$-1.36846085942992$

$-2.40808418625568$

$-2.80989584223919$
2.41711594292952

2.30584648592999

3.32069153083852

3.05234696405750

3.17978332745568

3.48573977544265

3.64931106865752

3.57217048059322

3.38196688787108

3.46390309117331

3.07940936265539

2.86225704026558

2.20346798991098

3.06550580909181

2.13150097587911

0.90987479482841

0.96484178799852

0.81946981317615

$-0.35144961956162$

$-0.42449723755521$

$-1.23715464869442$

$-0.89061079534237$

$-1.75951009560537$

$-0.02932190636301$

$-1.09890279325563$

$-0.58407888425408$

0.32529402298097

$-1.42404426992410$

$-0.77118976474258$

3.45345674412339

3.52502543506323

4.43323050223820

2.72644512973837

3.14450713398349

2.36805564865576

4.11013998937419

3.18176889379525

$-3.33776159743127$

$-3.28570668322703$

$-3.01057990994247$

$-4.38439247795183$

$-2.46433977946824$

$-2.02253520211818$

$-1.93190298068224$

$-3.53921041043103$

$-3.22922343396348$

$-4.28804955076861$

$-2.83017351864086$

$-3.17207699277434$

3.79814038011297

$-0.85065654283391$

3.49800738059134

3.16865479515922

1.70339568936120

4.01085852867888

3.73465500693470

3.34231728789947

3.45819565682752

3.83492038654155

5.09621349908221

4.82061087534645

4.33320848016763

4.45710671762304

3.92301883379900

5.33197423886994

0.77655852985404 


\begin{tabular}{|c|c|c|}
\hline \multicolumn{3}{|l|}{$E=-5572.62485894 E_{h}$} \\
\hline Co 0.02572864805233 & 0.00066014740165 & -0.06468812728447 \\
\hline Co 0.03426679248064 & -0.09426955154522 & 2.46307883782677 \\
\hline P 0.07537890741236 & -0.92368374841308 & -2.11521754406673 \\
\hline P 0.09915734684358 & -1.14249849254948 & 4.46674625271788 \\
\hline N -1.89977019079068 & 0.18821954682111 & -0.18900436530850 \\
\hline$N-0.05272510568648$ & 1.80255367606860 & -0.41543392589440 \\
\hline N 1.92569132402961 & 0.35156622456914 & -0.19532242977551 \\
\hline N 1.93395249074876 & 0.23701412898005 & 2.61237953700567 \\
\hline N -0.03169740893811 & 1.69563574831629 & 2.89040383053700 \\
\hline$N-1.88518548460354$ & 0.09483515250091 & 2.62529424248780 \\
\hline C -2.33841628576932 & 1.40092018999051 & -0.45406289099538 \\
\hline C -1.27080890078648 & 2.39339231842721 & -0.52495765545586 \\
\hline C -1.35780375426421 & 3.78251468892415 & -0.70123642142490 \\
\hline H -2.33381823060452 & 4.27333947070962 & -0.77673235767498 \\
\hline C -0.16974027953752 & 4.53104245645932 & -0.77263912515101 \\
\hline C 1.07806571086284 & 3.88667357623007 & -0.70546716724553 \\
\hline H 2.00872395377420 & 4.45836704955929 & -0.78457619017972 \\
\hline C 1.11040901745231 & 2.49517510030991 & -0.52839233216210 \\
\hline C 2.25922796708189 & 1.59754350238075 & -0.45982203664014 \\
\hline C 2.93654838392753 & -0.71269249141648 & -0.11722851531916 \\
\hline H 3.61095007824366 & -0.65935078834199 & -0.99141636101154 \\
\hline H 2.39979941513444 & -1.67252585770739 & -0.16701567880897 \\
\hline C 3.77083962728178 & -0.66269453388419 & 1.16265869100008 \\
\hline H 4.49198687966623 & -1.50193420903626 & 1.12689376282803 \\
\hline H 4.36695005643101 & 0.26561527456569 & 1.19872150432217 \\
\hline C 2.94515643568667 & -0.81677023327577 & 2.43877094123346 \\
\hline H 2.40866842987169 & -1.77608630891965 & 2.41400199765619 \\
\hline H 3.62390198749255 & -0.83102923267899 & 3.31110108146665 \\
\hline C 2.27764151265780 & 1.47113876059116 & 2.92420462165760 \\
\hline C 1.13618843594626 & 2.37562107204582 & 3.02397055954480 \\
\hline C 1.11269864291095 & 3.76160630104687 & 3.24403607179401 \\
\hline H 2.04542959840270 & 4.32732253626700 & 3.34049330187212 \\
\hline C -0.13163224972404 & 4.41002176395913 & 3.33555679925905 \\
\hline C -1.32487594704870 & 3.67078509440218 & 3.24930850903702 \\
\hline H -2.29707194157652 & 4.16492080923072 & 3.34886745602568 \\
\hline C -1.24602144901239 & 2.28699533287257 & 3.03035175963674 \\
\hline C -2.31774130559824 & 1.30004609999108 & 2.93683176854292 \\
\hline C -2.81491631724217 & -1.03467814260022 & 2.46377304803142 \\
\hline H $\quad-3.49474512360470$ & -1.08693819436469 & 3.33283652574236 \\
\hline H -2.20610996814322 & -1.94963515544501 & 2.46041526119350 \\
\hline C -3.64757864473112 & -0.96825568539052 & 1.18389279549136 \\
\hline H -4.33181689086735 & -0.10185842979674 & 1.20808137540395 \\
\hline H $\quad-4.28834745565248$ & -1.87126718671235 & 1.15867923965050 \\
\hline C -2.81928374370793 & -0.95491383263341 & -0.10158677665951 \\
\hline H -2.20774334394776 & -1.86877607896520 & -0.15656874963977 \\
\hline H $\quad-3.50199896253167$ & -0.95543003975343 & -0.97042148642195 \\
\hline C -3.75651898846737 & 1.79705655024588 & -0.73769859461729 \\
\hline H -3.79118200525349 & 2.41544721137273 & -1.65565935843228 \\
\hline H $\quad-4.16259136717068$ & 2.42320822952327 & 0.08201487851763 \\
\hline H -4.42772324701693 & 0.93539587461080 & -0.87598637194178 \\
\hline C 3.63661784136053 & 2.11594407401955 & -0.74560598844563 \\
\hline H 3.61872276148368 & 2.71903037302385 & -1.67444888056807 \\
\hline H 4.38687317405226 & 1.31967500262069 & -0.86592797025892 \\
\hline H 3.97620448793477 & 2.79263974880099 & 0.06414166997123 \\
\hline C 3.66719247305093 & 1.96391702647809 & 3.19630399880306 \\
\hline H 4.37453382613786 & 1.14868764674323 & 3.41843697323991 \\
\hline H 3.65671137825757 & 2.65710499343225 & 4.05842299206797 \\
\hline H 4.06326200887288 & 2.53377637345597 & 2.33188042242167 \\
\hline C -3.73965986370507 & 1.68990231987444 & 3.20998331020956 \\
\hline H -4.16389883674882 & 2.25676242484593 & 2.35699197341087 \\
\hline H -3.78313648465516 & 2.35789518989575 & 4.09105335004899 \\
\hline
\end{tabular}




\begin{tabular}{|c|c|c|c|}
\hline & & & \\
\hline & 308400253362 & 7068839141320 & \\
\hline & 19 & 19330 & \\
\hline & & & \\
\hline & 523 & & \\
\hline & & & \\
\hline & & & \\
\hline & & & \\
\hline & & & \\
\hline & & & \\
\hline & & & \\
\hline & & & \\
\hline & & & \\
\hline & & & \\
\hline & & & \\
\hline & & & \\
\hline & & & \\
\hline & & & \\
\hline & & & \\
\hline & & & \\
\hline & & & \\
\hline & & & \\
\hline & & -2.3 & \\
\hline & -1.6 & -2.8 & \\
\hline & -2.2 & -1.3 & \\
\hline & & & \\
\hline & & & \\
\hline & 2.02 & -5.5 & \\
\hline & 1.065 & -5.70 & \\
\hline & & & \\
\hline & & & \\
\hline & 0.63 & -5.5 & \\
\hline & 0.31 & -5.7 & 3 \\
\hline & -0.278380 & -4.9 & 1.8 \\
\hline & -0.4436 & -6.8 & \\
\hline & -1.1 & -5.3 & \\
\hline & -1.81 & -5.3 & \\
\hline & & -6.4 & \\
\hline & & & \\
\hline & & & \\
\hline & & & \\
\hline & & & \\
\hline & & & \\
\hline
\end{tabular}

\begin{tabular}{|c|c|c|}
\hline \multicolumn{3}{|c|}{$\left[\left({ }^{3} \mathrm{PDI}_{2}\right) \mathrm{Co}_{2}\left(\mu-\mathrm{NPMe}_{3}\right)\left(\mathrm{PMe}_{3}\right)\right]^{3+}\left({ }^{3}<\mathrm{Co}_{2} \mathrm{NP}>^{3+}, \mathrm{S}=0\right)$} \\
\hline Co -0.09626044610567 & -0.07213054967197 & 0.08835440107970 \\
\hline Со -0.13846190292101 & 0.19134543684583 & 2.52947374555337 \\
\hline 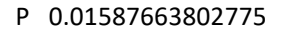 & -1.00988095792231 & -1.95062781765200 \\
\hline$N-2.03895255712552$ & 0.13625547600816 & -0.08476642066549 \\
\hline$N-0.14504973016651$ & 1.67978657501711 & -0.47370981370638 \\
\hline N 1.82453373651971 & 0.25658393961736 & -0.00584972143024 \\
\hline N 1.76389899543005 & 0.51320969230780 & 2.79445171612705 \\
\hline$N-0.20550089136523$ & 1.98689045567297 & 2.90456653411399 \\
\hline N -2.06578827304422 & 0.38470225110959 & 2.72076091856898 \\
\hline$N-0.07602356351257$ & -1.40768112126767 & 1.45806716401270 \\
\hline C -2.44300458956943 & 1.31514744580457 & -0.48772557293596 \\
\hline C -1.34856664026720 & 2.27428647525926 & -0.65351464982666 \\
\hline C -1.41464588591945 & 3.64368245735096 & -0.95063375453094 \\
\hline H $\quad-2.38257312276337$ & 4.135723 & -1.08932 \\
\hline C -0.21679500387632 & 4.370278 & -1.059806 \\
\hline C 1.01857212116171 & 3.72165746005793 & -0.89381979377142 \\
\hline H 1.95886202142946 & 4.27424712877904 & -0.98869713351603 \\
\hline C 1.02 & 52206 & -0.59888705016696 \\
\hline 16076070208660 & 1.46144280449379 & -0.38923571076336 \\
\hline
\end{tabular}


C 2.82707191993808

H 3.51832309242867

H 2.27609010277474

C 3.62497407287482

H 4.33926949919497

H 4.23011798973005

C 2.77372954415136

H 2.23402219689736

H 3.42777448626154

C 2.09933097255915

C 0.95577053192845

C 0.94058262908709

H 1.87689020488304

C -0.30041894313967

C -1.49384365094001

H -2.46675395638389

C -1.41300318762551

C -2.49199890923132

C -3.00015799911016

H -3.67703032709962

H -2.39962637122846

C -3.82027570437813

H -4.48515289945554

H -4.47903270732990

C -2.98803225590902

H -2.39131924390362

H -3.66654614454999

C -3.85206830613217

H -3.87948297219571

H -4.23995203306923

H -4.53860686422637

C 3.55988023530973

H 3.87722474012122

H 3.58193013520549

H 4.30156491614556

C 3.46390710717912

H 4.20945187587637

H 3.41048651714249

H 3.82976364591009

C -3.89838242427683

H -4.28723884005695

H -3.91567226616110

H -4.58880799503837

C -1.57373846683349

H -2.14664869526960

H -2.16517015682592

H -1.35992104597471

C 0.82166118404333

H 0.26072274393121

H 1.86404089134940

H 0.81651339910915

C 0.94574638783789

H 0.92727695757258

H 1.99436669107800

H 0.47570357867878

H -0.33803752480182

H -0.24579068583937

H 1.60896787600824

C 1.47865339173240

H -1.33690723734535

H 1.35564676253112

H 2.38053959189765

C -1.33983515880953

H -1.16636318590065

P -0.01535156313229

H -2.32397419570554
$-0.78531910660132$

$-0.85124403767733$

$-1.72836308094629$

$-0.57764935157933$

$-1.41925512963259$

0.34390037994505

$-0.55034098894595$

$-1.50013515121345$

$-0.43898234143084$

1.75946337777303

2.67805948727069

4.07848094672380

4.64293266449523

4.73897885214331

3.99680573671092

4.49852716708389

2.59854648900847

1.60450271853095

$-0.74625045251546$

$-0.69341657120309$

$-1.65907558253314$

$-0.81631236367453$

0.06288116148864

$-1.70395950696751$

$-0.96189611579675$

$-1.87935933414003$

$-1.04485487265305$

1.72370908766913

2.30250950469920

2.39109176919271

0.86786065724632

1.95628252438072

2.64860264142329

2.53481772315080

1.14587402838685

2.28743141467478

1.49913138068057

2.93958615036152

2.92350560933570

2.03622448599967

2.63995683095248

2.69447697631771

1.19635152374463

$-1.27662228032492$

$-0.33470666080735$

$-2.04117658885518$

$-1.63559764743494$

$-2.63809568011320$

$-3.37082112401115$

$-2.58017849921313$

$-2.96568342914562$

0.02729787250775

$-0.45867602693278$

0.13710067938290

1.02421056434233

5.83337371858117

5.44290986612472

$-3.95266295722690$

$-3.90059871092728$

$-3.96733862989863$

$-4.93007577014385$

$-3.45788911168547$

$-4.00167658240404$

$-5.04551971484485$

$-2.93815909499987$

$-3.67048939440509$
0.25057523468526

$-0.61018790804196$

0.31830590323306

1.54218732234359

1.62662189167038

1.47478697074423

2.81541376689361

2.92309194070081

3.69903771211646

3.03061297030400

2.99804969040899

3.07565140543949

3.13590263607737

3.07328469247241

3.03265427524468

3.05983444726021

2.95560738704185

2.94875249669937

2.70392725488948

3.57589257787025

2.80574443928223

1.41330828140000

1.33860904696030

1.47754275142282

0.13576162266010

0.18998256768837

$-0.73189598593460$

$-0.78679640509804$

$-1.72958353286691$

0.00906906285735

$-0.87947178347192$

$-0.64017791079779$

0.16548708551201

$-1.58343689057949$

$-0.70914652568628$

3.34535742061753

3.53067895890800

4.23896490303680

2.51345511210844

3.22382288581457

2.37821290838345

4.11438942199786

3.39547190265494

$-2.79738704879386$

$-2.85588218645115$

$-2.26685230084998$

$-3.82227906032937$

$-2.05281863897540$

$-1.44795647063033$

$-1.70011861167195$

$-3.11034200222469$

$-3.12459188171507$

$-4.11818719203794$

$-2.79958334972527$

$-3.20149648608612$

3.11950653668753

$-1.28207749347997$

0.35851133528605

1.45286539431559

0.11425822907058

1.83967906879684

1.90972771809429

1.21927607897831

1.54131440872390

1.87959274802838

1.59286824004941 


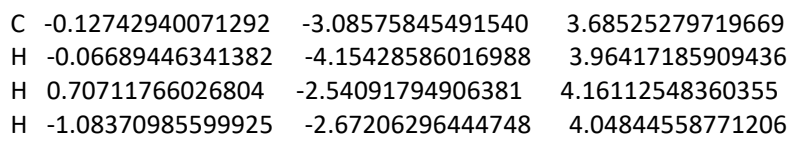




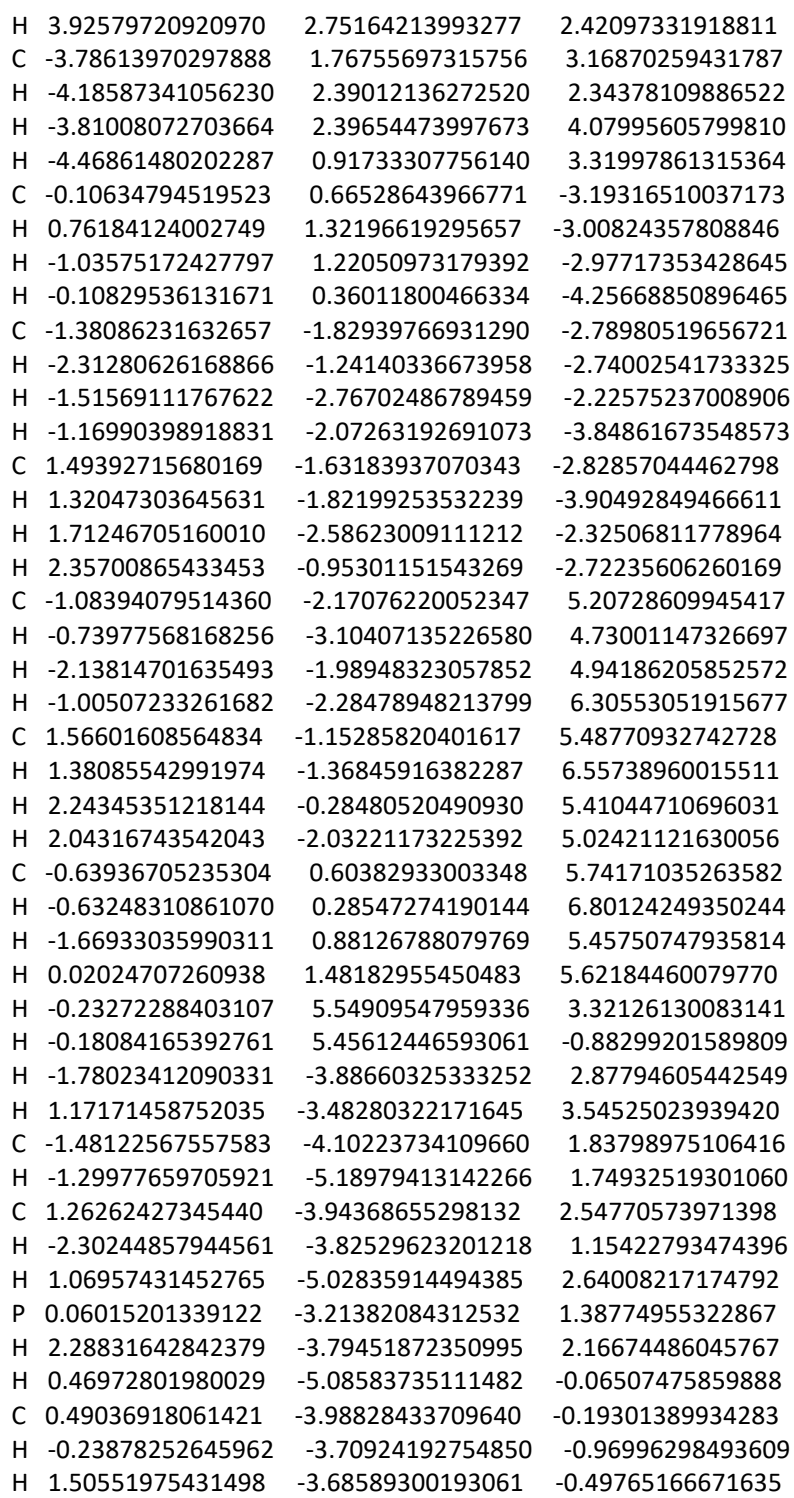

\footnotetext{
trans- $\left[\left({ }^{3} \mathrm{PDI}_{2}\right) \mathrm{Co}_{2}\left(\mu-\mathrm{NPMe}_{3}\right)\left(\mathrm{PMe}_{3}\right)_{2}\right]^{3+}\left(\right.$ trans $\left.-{ }^{3}\left[\mathrm{Co}_{2} \mathrm{NP}\right]^{3+}, \mathrm{S}=0\right)$ $\mathrm{E}=-5463.13298826 E_{\mathrm{h}}$

Co $-0.14366245418109 \quad 0.09037270834607 \quad 0.26522760609878$

Co $-0.12270321119034 \quad 0.70992384448246 \quad 3.24982233034604$

$\begin{array}{llll}\text { P } & 0.06541415761528 & 0.98543056312190 & -1.78260089877036\end{array}$

$\begin{array}{llll}\text { P } & 0.58324440226344 & -1.21895383695131 & 4.20220064745289\end{array}$

$\begin{array}{llll}\text { P } & -0.68796295716604 & 3.18632389829899 & 1.48432376984639\end{array}$

$\begin{array}{llll}\text { N } 1.77924778480448 & -0.08145418874223 & 0.33703399848746\end{array}$

N $0.03973292864082 \quad-1.65498209437221 \quad-0.37281289760847$

N $-2.03028328507629 \quad-0.35377871952719 \quad-0.07540518370829$

$\begin{array}{llll}\text { N } & -1.98850631995480 & 0.03858225809773 & 3.41099914394831\end{array}$

$\begin{array}{llll}\mathrm{N} & -0.66330813282888 & 1.62337950980368 & 4.79177338302350\end{array}$

$\begin{array}{llll}\mathrm{N} & 1.62458551615872 & 1.68968935510087 & 3.75542093366125\end{array}$

$\begin{array}{llll}\mathrm{N} & -0.41444200036535 & 1.61189884831710 & 1.49813819240216\end{array}$

C $2.29918752109759-1.22391382262586 \quad-0.07470712577655$

C $1.29198443093962 \quad-2.17622558858992 \quad-0.51190641995073$

$\begin{array}{llll}\text { C } 1.47101096302083 & -3.44101559313632 & -1.09393579596235\end{array}$

H $2.47308552349538 \quad-3.87357085728334 \quad-1.18039722078714$

$\begin{array}{llll}\text { C } & 0.34991134994157 & -4.13241394495022 & -1.57911788270465\end{array}$

C $-0.92287358014540 \quad-3.54134086021112 \quad-1.50332406829100$
} 
H -1.80231799657480

C -1.05236036826736

C -2.25740820507055

C -3.14116416532703

C -2.64019824833589

C -2.66105226300044

C -1.92481710064190

C -2.38120660552456

H -3.40376930475705

C -1.51328313792785

C -0.20851387095184

H 0.48529247477699

C 0.19891181804278

C 1.50341905588828

C 3.00685043026254

C 2.64641270929567

C 3.76202600644273

H 3.98518107062525

H 4.38368678727216

H 4.07757504208619

C -3.58642772192238

H -3.48917115092721

H -3.92964372964356

H -4.36541322340432

C -4.04591418848647

H -4.05335484458904

H -4.74411783659394

H -4.44213103121538

C 2.62132743534911

H 2.27224351049857

H 3.41892571949023

H 3.09316273410936

C -1.50467625124564

H -2.14354622913590

H -1.28760172645215

H -2.04472524560342

C 1.21712935941933

H 2.24728575538482

H 1.07728166207301

H 1.08129177880882

C 0.76458159029744

H 1.76444046317786

H 0.09635317283939

H 0.85765310694103

C 0.93421177466552

H 1.75080018201366

H 1.23129259299444

H 0.03200759334994

C -0.62613437010438

H -0.92430174876314

H -1.52077726640417

H -0.16364029826748

C 2.07766872443892

H 2.96595100239079

H 1.92962584198424

H 2.24640004159901

C -1.80982746078813

H -2.16259595411038

H -2.67362986449364

H -1.28991756817195

C 0.74892917898202

H 0.40574489347581

H 1.26432523596822

H 1.45868235275483

C -1.51264735207726

H -1.64617719884970
$-4.05064451387260$ $-2.28577235293114$

$-1.49249595277204$

0.41020042859630 $-0.98012843529521$ 0.48549580558641 1.43234940306549 2.09951180028080 1.94358739088268 2.97215470717275 3.14308976786838 3.81296923256107

2.44754159501521 2.46848479947101 1.63684687796293 0.99091931408351

$-1.54188429755426$ $-2.22829797691432$ $-0.63789178947414$ $-2.04814641537239$ $-1.99674974955095$

$-2.38029211484770$ $-2.84462330845334$

$-1.21768938422496$ 0.08622566599668 $-0.13805688823559$ 0.93218654847158 $-0.78986829034194$ 3.29185552223938 3.95206960463663 2.64112007874306 3.92261383338999 1.43099664452687 2.05761662412337 1.96087368856903 0.49422221070792

2.39259530187963

2.00122127694787

2.84946281146356

3.16197847113774 $-0.19862684954450$ $-0.53674262914975$ $-1.07030294284185$ 0.31084552721855

$-2.61167723480766$ $-2.33943942416604$ $-3.50640272952243$ $-2.83870362425221$ $-1.92167715451292$ $-1.14835145863142$ $-2.28068492296369$ $-2.76981783747957$ $-1.08456392472920$ $-0.84981603792700$ $-0.28457109552165$ $-2.04527612465419$ 3.74927417815038 4.76706551703559 3.06571061419696 3.78644519907072 4.29922769178237 5.35114218096259 4.09784315680073 4.15108529586375 3.81786319625767 4.90794261325852
$-1.91079954121659$

$-0.89294515400775$

$-0.69975846392885$

0.48597442184924

2.57636937340103

4.44885647830082

5.26153743796023

6.40793662370366

6.76548258345835

7.07776382357147

6.59532629600739

7.11161071836053

5.44462063473638

4.81337784615605

3.22834568607932

0.80099566665648

$-0.12099849831781$

$-0.95730895228652$

$-0.23847570910532$

0.81469134477594

$-1.17414849181735$

$-2.20850306682633$

$-0.54747324489789$

$-1.16651430840276$

4.87106084699593

5.95481644346731

4.70893886980186

4.33790463075646

5.38214142825109

6.19124413626170

5.79511728914319

4.60503742451431

$-2.60429080211284$

$-1.96534568254471$

$-3.55136961275286$

$-2.83052854593802$

$-1.96921089960844$

$-1.89681109081982$

$-2.96754332052821$

$-1.19407520477762$

$-2.98486794223727$

$-2.66229687276495$

$-3.09563114714037$

$-3.96289190762715$

3.07232269856700

2.38171374926367

3.65310449921912

2.47761792630555

5.37807933321235

6.10946066786712

4.84260823891906

5.91727908021206

5.24425125932085

4.63496501760254

5.99288891836502

5.76742949720973

2.80206991267973

2.55341244515492

2.86654849838089

3.77171449183613

1.66018441638412

1.65424955352168

2.61418544900838

0.82633289733791

$-0.00918170486171$

0.11663558885078 


$\begin{array}{clcc}\text { H } & -0.91167049388444 & 3.65109217344720 & -0.91430970487066 \\ \text { H } & -2.50503246565215 & 3.35036004317349 & -0.12378804009654 \\ \text { H } & -1.84748355707500 & 3.51083117152677 & 7.97157591609568 \\ \text { H } & 0.47019657488150 & -5.12049081675054 & -2.03782649441618 \\ \text { H } & -3.91914596783196 & 0.62200681122127 & -0.27071912346904 \\ \text { H } & -2.72247595515029 & 1.35767135838164 & 0.84793794216558 \\ \text { C } & -3.72289757991745 & -0.39083394902687 & 1.66036709188057 \\ \text { H } & -4.33305391945675 & -1.23263759963210 & 1.28553273059950 \\ \text { H } & -4.40461683257901 & 0.26528732988417 & 2.23086332640540 \\ \text { H } & -3.08764448239214 & -1.75287151333082 & 3.22530806860313 \\ \text { H } & -1.86090885874518 & -1.48107188601353 & 1.98615954244917 \\ \text { C } & 3.28002311638236 & 0.61792295956512 & 2.14920503793425 \\ \text { H } & 2.01144009188733 & 1.87975741631227 & 0.90682809040133 \\ \text { H } & 3.41902178217305 & 1.21020098715488 & 0.04082248416154 \\ \text { H } & 4.37698530720284 & 0.53358985429416 & 2.05089485407821 \\ \text { H } & 2.91893947114616 & -0.37401634520391 & 2.46884671909631 \\ \text { H } & 3.68278217889976 & 1.43006770692384 & 4.07815582114823 \\ \text { H } & 3.27800138297177 & 2.64283370346093 & 2.85150924722005\end{array}$

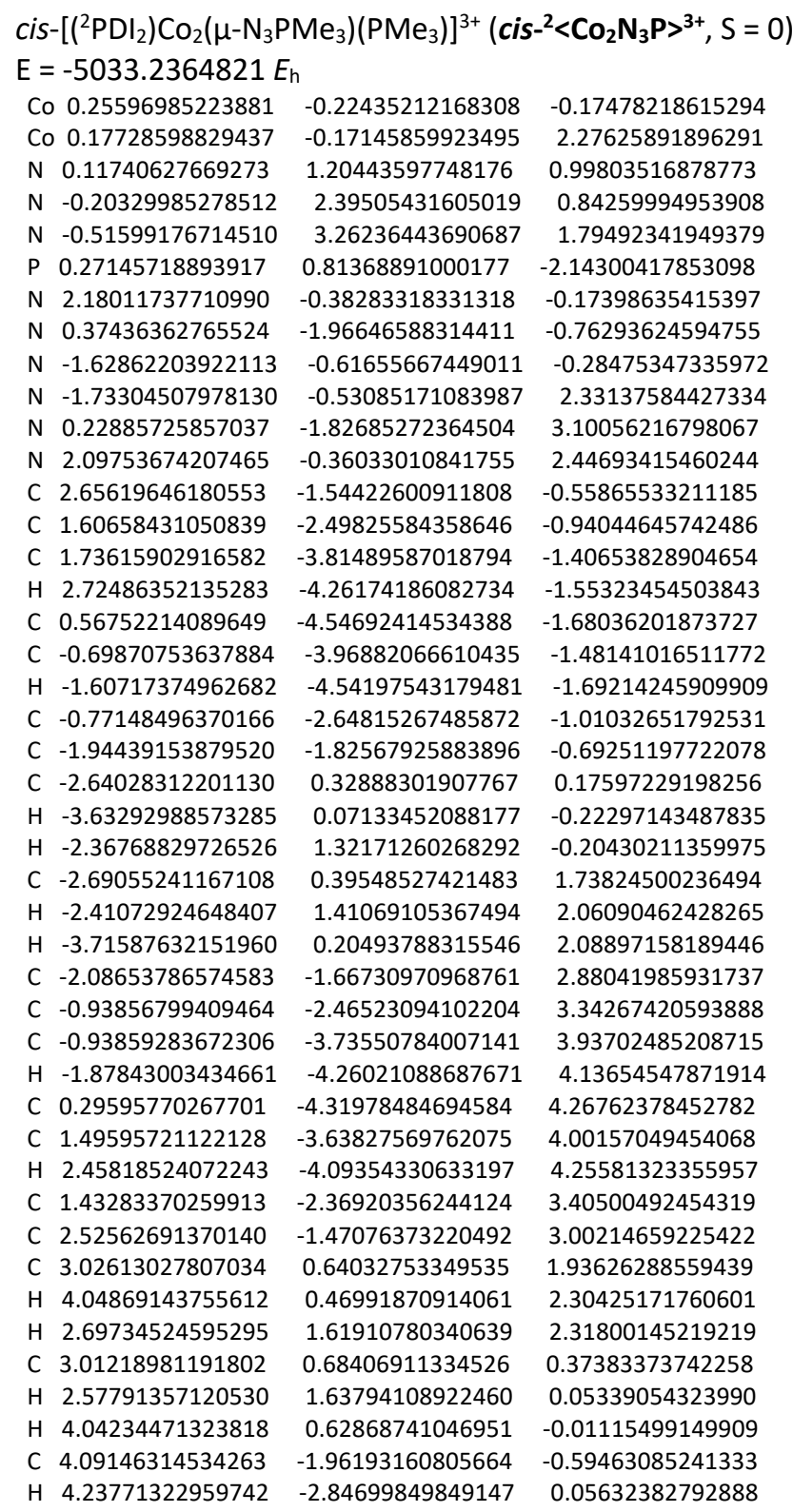




\begin{tabular}{|c|c|c|c|}
\hline & & & \\
\hline & & & \\
\hline & & & \\
\hline & 816 & 10 & \\
\hline & 211 & & \\
\hline & 9569778 & -1.807802 & \\
\hline & & -2.21080469 & \\
\hline & 671 & -2.39 & \\
\hline & 6184 & -3.1 & \\
\hline & 3639 & 00 & \\
\hline & 9629126 & 182860 & \\
\hline & 747 & -1.2 & \\
\hline & 8 & 1.6 & \\
\hline & 00 & -2.89 & \\
\hline & 720 & 52 & \\
\hline & 005 & 2.00636 & -3. \\
\hline & 3 & 506 & -1 \\
\hline & 80 & & \\
\hline & 6 & 88 & \\
\hline & 208525 & 0.20 & -4.4 \\
\hline & $-0.0 \mathrm{~s}$ & -1.13 & \\
\hline & 1.65 & -0.8 & -3. \\
\hline & & & \\
\hline & & & \\
\hline & 1.3 & 2.8 & -1 \\
\hline & 1.3 & 0 & -3.2 \\
\hline & & & \\
\hline & & & \\
\hline & -2.4 & 3.8 & \\
\hline & -1.4 & & \\
\hline & -1.4 & & \\
\hline & -1.2 & & \\
\hline & -0.2 & & 3.4 \\
\hline & -1.3 & 5 & 4 \\
\hline & -0.31 & & \\
\hline & -0.0 & & \\
\hline & 1.4 & & \\
\hline & 1.40 & 3.8 & \\
\hline & 1.5 & & \\
\hline & & & \\
\hline & 19908040493048 & 3.24449469369750 & 3.3889997693985 \\
\hline
\end{tabular}

\begin{tabular}{|c|c|c|c|}
\hline \multicolumn{4}{|c|}{$E=-5033.24219180 E_{h}$} \\
\hline Co & -0.10325056252190 & -0.19717090094947 & 0.00982524105205 \\
\hline Co & -0.32864232709316 & -0.32276049139485 & 2.44937521506138 \\
\hline $\mathrm{N}$ & -0.57631885843892 & 1.07611391333182 & 1.23680585081453 \\
\hline $\mathrm{N}$ & -0.89447446788837 & 2.26897790399522 & 1.24150799196319 \\
\hline $\mathrm{N}$ & -1.17277665863347 & 2.83818501946114 & 2.43841550391857 \\
\hline $\mathrm{P}$ & -0.20427928366052 & 0.94652214112648 & -1.89069194069279 \\
\hline $\mathrm{N}$ & 1.80476886305028 & 0.03348886709891 & 0.15926635323849 \\
\hline $\mathrm{N}$ & 0.41276869160739 & -1.83722133560655 & -0.64540774586405 \\
\hline $\mathrm{N}$ & -1.86603493268541 & -0.95848460190098 & -0.23455763508895 \\
\hline $\mathrm{N}$ & -2.10003451834305 & -1.06611300832692 & 2.37622905766086 \\
\hline $\mathrm{N}$ & 0.03084886150220 & -1.95636211933880 & 3.22757624974562 \\
\hline $\mathrm{N}$ & 1.55132755150857 & -0.07101850816591 & 2.78007253367053 \\
\hline C & 2.53735252791728 & -0.98178048324916 & -0.24228854827644 \\
\hline C & 1.73818247240441 & -2.10243708363150 & -0.75334267194653 \\
\hline C & 2.15097336316449 & -3.34093241236509 & -1.27050445634089 \\
\hline $\mathrm{H}$ & 3.21378008182235 & -3.58220159757260 & -1.37285040989903 \\
\hline C & 1.17104395649831 & -4.27263003731658 & -1.65595706987498 \\
\hline C & -0.19508490882126 & -3.97203168904508 & -1.51282015364221 \\
\hline $\mathrm{H}$ & -0.96152532772075 & -4.69935165887011 & -1.80006484545299 \\
\hline C & -0.55320311916140 & -2.71951695148389 & -0.99248122170439 \\
\hline C & -1.89173191955602 & -2.18307521269163 & -0.70669219556478 \\
\hline
\end{tabular}


C -3.05764736919484

H -3.96610278904294

H -2.95556693871392

C -3.19322187944027

H -3.12454026340177

H -4.17592804022415

C -2.23429051721177

C -0.97247753266691

C -0.71672036697019

H -1.51886790477062

C 0.59511294231113

C 1.62036253765755

H 2.64471373546832

C 1.29997344758780

C 2.18144669972363

C 2.20876703546696

H 3.18404742319253

H 1.55231989782643

C 2.39453705351787

H 1.88045471447037

H 3.46248575126204

C 4.03255913727600

H 4.48387754496551

H 4.41367784682537

H 4.39290317749571

C -3.09594841976369

H -4.03673382342169

H -2.96923113943822

H -3.18870812100859

C -3.47495068983261

H -3.76955405299923

H -3.26866907587154

H -4.32892463187095

C 3.63242928819352

H 4.20643293819500

H 4.08814665314619

H 3.74150709078202

C -1.84612543484120

H -1.75789726854884

H -2.19947069214723

H -2.56561496422669

C 0.30029539168012

H 0.25883361522591

H -0.38317053386090

H 1.33182448847274

C 0.86168648037489

H 1.92410042312946

H 0.60490962958548

H 0.68811304556851

H 0.82019048563120

H 1.47524370297908

H $\quad-0.04172277664517$

H 0.64526010800792

C -0.25969567574961

H -0.57080144239829

P -1.58191570631027

H -2.85128222253926

C -3.05087492665606

H -3.29201509659664

H -1.00711740339853

C -1.91492641098108

H -2.19564939561468

H -3.90008986491463

H -2.74469839969747
$-0.24973704748283$ $-0.65194791275555$ 0.79957983523976 $-0.30455367259725$ 0.71712147770545 $-0.71737058979048$ $-2.29318057692683$ $-2.85633742787805$ $-4.13302481471819$

$-4.87278699194394$

$-4.44119325500693$

$-3.48593400790670$

$-3.71979153971317$

$-2.22755092646961$

$-1.07564836952145$

1.16086274448888

1.29653731152965 1.99028139222636

1.20191945884511 2.09232613342375

1.29121725563508

$-1.03353059278407$

$-0.29601940127714$

$-2.03166178324808$

$-0.79338606163179$

$-3.04541891065873$

$-2.57782662670618$

$-3.99923640159247$

$-3.30607200242662$

$-3.12686922008259$

$-3.34917852368535$

$-4.10181619310761$

$-2.65670197139856$

$-1.16560727787734$

$-0.25636875167771$

$-2.02237751628411$

$-1.37813193667229$

1.58101942696000

2.10723069531548

2.29600679909423

0.75071581523940

$-0.09239098142631$

0.49828580700104

$-0.95672479336387$

$-0.45562278811064$

2.41548913486384

2.12476608586550

3.10985764998645

2.92122221800469

$-5.43233102748370$

$-5.24262298234259$

5.03801821784740

5.33907826099764

5.42388595772081

6.48317586163576

4.43403846753757

4.26869479406869

4.67195599155778

5.74911131159263

4.91091226182814

5.03124515810044

6.09950260322439

4.12824953326252

4.44660546264589
0.22720363248960

$-0.24648644805173$

$-0.07783007806460$

1.78653513172745

2.19411689237207

2.06221250153421

2.81964487569070

3.33368322366686

3.85583114580981

3.94507446111791

4.25957119325313

4.13614156786923

4.44410103295523

3.60533640621012

3.34006864533623

2.36494249933383

2.85749216335645

2.67449596951082

0.80925761440005

0.42582747269058

0.55836180108730

$-0.16382550089977$

$-0.85794403376130$

$-0.43452143282765$

0.85371812251922

$-0.91460057525326$

$-0.58749482322607$

$-0.36524684650773$

$-1.98835938684750$

2.82642508211219

3.87253031589970

2.34143138553754

2.31476545124598

3.68631380120543

3.45030086360966

3.14970128153714

4.76905920461773

$-2.34477575630215$

$-3.31491079299901$

$-1.58018626406955$

$-2.44854320844578$

$-3.29401183905384$

$-4.22888031530339$

$-3.37796230747854$

$-3.13674946574893$

$-1.97373107857489$

$-1.90419257702837$

$-1.15310030814678$

$-2.94300032692378$

4.67001029591375

$-2.06524718459539$

0.50934298228626

2.15349238281470

1.52314350694210

1.45457404487445

2.25217073355427

0.21976900540319

1.23058813091630

1.15543314518455

4.52829308593317

3.90897333594878

3.86024666170184

1.68475373030269

4.34659066686061

$\left[\left({ }^{2} \mathrm{PDI}_{2}\right) \mathrm{Co}_{2}\left(\mu-\mathrm{NPMe}_{3}\right)\left(\mathrm{PMe}_{3}\right)\right]^{3+}\left({ }^{2}<\mathrm{Co}_{2} \mathrm{NP}>^{3+}, \mathrm{S}=0\right)$ 
$\mathrm{E}=-4923.80005075 E_{\mathrm{h}}$

Co 0.11787065914961 Co 0.14278026088674 N -0.13881387048426

P -0.06891736159257

N 2.04408999803631

N 0.44128316442242

N -1.71160174883718

N -1.70539272901570

N 0.40869094887210

N 2.07744903194272

C 2.65452392771605

C 1.72537183784132

C 1.99329530381319

H 3.02093829702183

C 0.91819709060763

C -0.39967188799923

H -1.24396496783129

C -0.61227337404447

C -1.87476641595817

C -2.80002890849779

H -3.77247450393258

H -2.62663385315413

C -2.81472101900540

H -2.69554728796185

H -3.78310325356019

C -1.91295628160189

C -0.66828008313762

C -0.48561455416022

H -1.34297127976173

C 0.82322664725124

C 1.92129814735721

H 2.94474107734931

C 1.67609205875419

C 2.64635546009781

C 2.84422811659371

H 3.89052356838996

H 2.38796113906280

C 2.78690976888406

H 2.26139985757463

H 3.80848099281376

C 4.14162789804095

H 4.58338292959258

H 4.41765402089659

H 4.61493819969475

C -3.15551039459241

H -4.03579963874729

H -3.07893687882220

H -3.32530577085486

C -3.25702231081931

H -3.16262589924085

H -3.83781611005642

H -3.85044221033273

C 4.10504299319611

H 4.76028433091198

H 4.39147864742849

H 4.30193706024183

C 0.83573754047826

H 0.74988755439351

H 1.90204391400940

H 0.39048328404412

C -1.76526731750327

H -1.72470228750229

H -2.22447482662464

H -2.37773519206392

C 0.57029433142638
$-0.12179461380971$ $-0.45914109465152$ 1.14073445772636 1.00343238485062 $-0.03473586387845$

$-1.75379946793252$

$-0.76313035527548$

$-1.02275205113599$

2.17400996018845

$-0.42065521270953$

$-1.07031316017989$

$-2.10771734862575$

$-3.35000565168661$

$-3.65528902843424$

$-4.20084757701436$

$-3.81155563060901$

$-4.47059719914410$

$-2.55930339909262$

$-1.95053343193176$

$-0.00584954604953$

$-0.29777912621636$

1.04904816061103

$-0.18465821614102$

0.79855070635199

$-0.59814597335751$

$-2.25124616641197$

$-2.96860205039259$

$-4.28718823483534$

$-4.94339094023788$

$-4.75426221332533$

$-3.90625643122469$

$-4.25628306523554$

$-2.59809545846404$

$-1.53029179712274$

0.76353948547004

0.68936295637873

1.61340420474318

1.02209299071941

1.96433417554659

1.10691120218011

$-1.22225447186982$

$-0.38813258114103$

$-2.16605278513817$

$-1.21314466991414$

$-2.70740732188637$

$-2.17095092833326$

$-3.68162452571389$

$-2.93661411535979$

$-2.90092871029021$

$-3.96435687632059$

$-2.83952100126468$

$-2.39824392043426$

$-1.80520770746897$

$-0.96864831849768$

$-2.69806399700850$

$-2.05408346395232$

2.57578652387092

2.97582998494291

2.42610960596966

3.30196567777109

1.42841857507355

1.92781454382093

2.11677819837643

0.51322108470556

$0.02924501994263-3.09613436182587$
0.21101780643218

2.62258943663051

1.60288494001766

$-1.69901857865311$

0.15657939240249

$-0.56631776727952$

0.12223642205325

2.71732406298224

3.22628876495803

2.73777413944779

$-0.37294686061397$

$-0.82823211511422$

$-1.42462787454343$

$-1.64569808245053$

$-1.73215744211574$

$-1.43725649043045$

$-1.66440530438137$

$-0.84270077537731$

$-0.40767482404245$

0.72610776072282

0.30145921663495

0.48755036102849

2.27746732423419

2.75471386980940

2.59583735662021

3.13164064696556

3.44594936455244

3.89080335342744

4.07081696847405

4.10290707767722

3.87927987258553

4.04855976120038

3.43730419127199

3.14334126469431

2.37092615778530

2.89917996526902

0.83019257642344

0.64261550866663

0.42716995694568

$-0.47060045582894$

$-1.05056610367971$

$-0.96790547198232$

0.52985572545670

$-0.56154103084567$

$-0.17645550948806$

$-0.03870697676122$

$-1.63284355718898$

3.26982476465651

3.54264420727833

2.33063962614452

4.05971945301275

3.32516331552680

3.03898060529993

2.73432153767500

4.38786938663361

$-1.78613052860860$

$-2.81482495105970$

$-1.54540568102033$

$-1.08526856314656$

$-2.19838740862394$

$-3.18556001837931$

$-1.46884925857252$
2.70170598377898 


$\begin{array}{clcc}\text { H } & -0.01276694418001 & -0.90312027946193 & -3.19985568909602 \\ \text { H } & 1.63333970798645 & -0.21969227387778 & -2.93031876533436 \\ \text { H } & 0.47870894620711 & 0.62290571583573 & -4.02538430582348 \\ \text { H } & 0.98641254032333 & -5.78172195933918 & 4.44788255029004 \\ \text { H } & 1.10911982485445 & -5.17338841434530 & -2.19947140129822 \\ \text { H } & -1.76823290730572 & 3.51855128934312 & 0.34473071719929 \\ \text { H } & -2.77264384172212 & 2.89891545303646 & 1.71150255716256 \\ \text { C } & -1.85403921301965 & 3.45030933428173 & 1.44497107018929 \\ \text { H } & -1.92006062599610 & 4.47572165225207 & 1.85412862072761 \\ \text { P } & -0.38438762057242 & 2.62048259630056 & 2.12436040914715 \\ \text { H } & 1.10606679687127 & 4.02842801804875 & 0.78637462059824 \\ \text { C } & 0.91396923076729 & 3.87802983542525 & 1.86278704326971 \\ \text { H } & -1.44942562647360 & 1.94123274147478 & 4.20011650805708 \\ \text { H } & 0.56513519901200 & 4.83623371408155 & 2.29222974357636 \\ \text { C } & -0.59143453309320 & 2.57914755949568 & 3.92458712770168 \\ \text { H } & -0.76453091989030 & 3.60645552571153 & 4.29627870350949 \\ \text { H } & 1.85196408612202 & 3.58905123322645 & 2.36801659033891 \\ \text { H } & 0.32852805901210 & 2.17843397936697 & 4.38918088463310\end{array}$

$\left[\left({ }^{2} \mathrm{PDI}_{2}\right) \mathrm{Co}_{2}\left(\mu-\mathrm{NPMe}_{3}\right)\left(\mathrm{PMe}_{3}\right)\right]^{3+}\left({ }^{2}\left\langle\mathrm{Co}_{2} \mathrm{NP}\right\rangle^{3+}, \mathrm{S}=1,\left\langle\mathrm{~S}^{2}\right\rangle=2.431513\right)$ $\mathrm{E}=-4923.79401632 E_{\mathrm{h}}$

Co $-0.01871060735754 \quad 0.19161172700258 \quad-0.09910469225930$

Co $-0.09801516788572 \quad 0.40079868648123 \quad 2.40640561401562$

P $-0.24319857480348 \quad 1.09337505355361 \quad-2.12476124149012$

$\begin{array}{llll}P & -0.54938794700447 & 3.21199536577084 & 1.45104056102501\end{array}$

N $1.91043516982291 \quad 0.23685393231148 \quad-0.07191078782442$

N $0.31561529304495 \quad-1.50887018013785 \quad-0.71285064960251$

N $-1.82433259994346 \quad-0.49123355808344 \quad-0.09146508421050$

N $-2.13576928576533 \quad-0.09466662797083 \quad 2.84160478118282$

$\begin{array}{llll}N & -0.10869391130044 & 0.37150226432492 & 4.38982925776004\end{array}$

N $1.98568927508961 \quad 0.63217058050948 \quad 2.88313773758442$

N $-0.28729146876095 \quad 1.65765481310444 \quad 1.08798377412306$

C $2.52540457133620 \quad-0.81921610698013 \quad-0.56150295559294$

C $1.59901979010716-1.86658222816912-0.97766193260946$

C $1.87448215891499-3.12052865541283-1.54185485199209$

H $2.90584652382740 \quad-3.41549339588891 \quad-1.76028601870520$

C $0.80527126432608 \quad-3.99073199176149 \quad-1.81438433290831$

C $-0.51191743456555 \quad-3.60094755135944 \quad-1.52120923906031$

H $-1.35308365679636 \quad-4.27252132593610 \quad-1.72124580207548$

C $\quad-0.73371529677945 \quad-2.33314117260644 \quad-0.96348204314041$

C $-1.98999208945674 \quad-1.70928780760498 \quad-0.56396230157064$

C $-2.93744679171715 \quad 0.25582631446228 \quad 0.47978455152659$

H $-3.85120415143247 \quad 0.13670573386875 \quad-0.12821143124571$

H $-2.66177005975902 \quad 1.31472565657273 \quad 0.47113043810851$

C $-3.25966500732196 \quad-0.19265676370965 \quad 1.90390780931752$

$\begin{array}{llll}\text { H } & -4.11566387575091 & 0.41858484597056 & 2.24600667524061\end{array}$

H $-3.60325385240937 \quad-1.24571182457239 \quad 1.89456454844875$

C $-2.41551427286454 \quad-0.10044031955748 \quad 4.11509647168890$

C $-1.24934381399885 \quad 0.01277614658699 \quad 5.02036437099344$

C $-1.25279437961257 \quad-0.25643215219247 \quad 6.39906659335012$

H $-2.17252260375007 \quad-0.54308345329190 \quad 6.91652045265086$

C $-0.04387377289437 \quad-0.17945519310343 \quad 7.10475857410681$

C $1.13834128733029 \quad 0.13920670045689 \quad 6.42204291562032$

H $2.09144328492994 \quad 0.16343532743331 \quad 6.95833276521383$

$\begin{array}{llll}\text { C } 1.07510221377377 & 0.39923824885584 & 5.04275147045232\end{array}$

C $2.23037810442547 \quad 0.68614282277181 \quad 4.16261805862701$

C $3.09086224776153 \quad 0.96404537462797 \quad 1.97413238160216$

H $3.78417773222451 \quad 0.09990258225660 \quad 1.94622551808646$

H $3.66784575449566 \quad 1.82731928179377 \quad 2.35722776060512$

C $2.65701710863080 \quad 1.32153589636922 \quad 0.55303053849443$

H $2.01195512369130 \quad 2.20809919446630 \quad 0.56952645647914$

H $3.562074280480551 .57266062577006-0.02826725391368$

C $4.00448491528859-1.02744131931078-0.65172430704252$

H $4.26242266338760 \quad-1.47858857024643 \quad-1.62882925213574$

H $4.58100705044602-0.09658614667594 \quad-0.53599728178833$ 


\begin{tabular}{|c|c|c|c|}
\hline & & & \\
\hline & -3.28226655739755 & 2380 & \\
\hline & -3.3256 & -3.01196825754267 & \\
\hline & 3.34948519805727 & -3.20572131821724 & \\
\hline & -4.16629367600658 & -1.80151236596732 & \\
\hline & -3.80717036956849 & -0.22674603642316 & 4.663367 \\
\hline & -3.8271 & -0.2 & 5 \\
\hline & 5844 & & \\
\hline & -4.30383842651923 & -1.13163832 & 4.26 \\
\hline & 73 & 1.01 & \\
\hline & & & \\
\hline & & & \\
\hline & & & \\
\hline & & & \\
\hline & & & \\
\hline & & & \\
\hline & & & \\
\hline & & & \\
\hline & & & \\
\hline & & & \\
\hline & & & \\
\hline & & & \\
\hline & & & \\
\hline & & & \\
\hline & & & \\
\hline & & & \\
\hline & & & \\
\hline & & & \\
\hline & & & \\
\hline & & & \\
\hline & & & \\
\hline & & & \\
\hline & & & \\
\hline & & 4.3 & 0.2 \\
\hline & & & \\
\hline & & & \\
\hline & -0.3 & & \\
\hline & -0.0 & & \\
\hline & . & $92 \angle 14$ &  \\
\hline
\end{tabular}

\begin{tabular}{|c|c|c|c|}
\hline \multicolumn{4}{|c|}{$\left[\left({ }^{2} \mathrm{PDI}_{2}-\mu-\mathrm{NH}\right) \mathrm{Co}_{2}\left(\mathrm{PMe}_{3}\right)_{2}\right]^{3+}\left({ }^{2}\left[\mathrm{Co}_{2} \mathrm{NCH}\right]^{3+}, \mathrm{S}=0\right)$} \\
\hline \multicolumn{4}{|c|}{$\mathrm{E}=-4923.82054409 E_{\mathrm{h}}$} \\
\hline & 0.09534682519166 & -0.29803941469604 & -0.0044778528346 \\
\hline & 0.12071667616872 & -0.27685 & 2.591654 \\
\hline & -0.86058792701339 & 0.870722 & 43 \\
\hline & 0.10950813917899 & 1.075427 & -1.7 \\
\hline & 481812976 & 1.01 & \\
\hline & 2.05613652058118 & -0.41 & \\
\hline & 0.25768325829217 & -1.88 & \\
\hline & -1.6 & -0.7 & \\
\hline & & -1.1 & \\
\hline & 0.393 & -1.7 & \\
\hline & 1.769 & 0.05 & \\
\hline & 2.530 & .45 & \\
\hline & 1.488 & -2.3 & \\
\hline & 591 & -3.52 & \\
\hline & 23 & -3.92 & \\
\hline & & -4.1 & \\
\hline & & -3.6 & \\
\hline & & -4.21 & \\
\hline & & -2.51 & \\
\hline & & $-1.7 \mathrm{c}$ & \\
\hline & & & \\
\hline & & & \\
\hline & & & \\
\hline
\end{tabular}


H -3.26980728444492

H -3.70377472545034

C -1.88017066997852

C -0.57558375812928

C -0.21463837460928

H -0.96956990527927

C 1.13278732002025

C 2.10411673310351

H 3.15442372781658

C 1.70275088384298

C 2.49803590942398

C 2.36928855130258

H 3.14858938259826

H 1.57126563263677

C 2.97167409775455

H 3.14727551015953

H 3.95099988029452

C 3.98979706387959

H 4.49124647759347

H 4.14264646297504

H 4.50335128345209

C -3.48382105570401

H -4.19726762142276

H -3.66187754727754

H -3.69426145503344

C -3.15904734616597

H -3.01979394157781

H -3.53619208571479

H -3.94128696734956

C 3.98498792503855

H 4.40580556345815

H 4.46878698584510

H 4.26653451508070

C 0.44686009781808

H 0.47980953309726

H 1.41819115429986

H -0.35392445799428

C -1.46912232486101

H -1.37816502144003

H -2.27269716670730

H -1.72265546982631

C 1.34481127736166

H 1.19226016329210

H 2.36380122246476

H 1.22814847038711

C -1.71654014057709

H -1.44672377791047

H -2.70129154357511

H -1.77497872173634

C 1.03030058856251

H 0.76037207329401

H 1.81490238358068

H 1.41165080376062

C -0.96248433772300

H -1.07065213667231

H -1.93084918116769

H -0.19963940395826

H 1.42841708134592

H 0.47906823485134

H $\quad-0.86278073632069$
0.39697147005108

$-1.02668079868337$

$-2.24179195482721$

$-2.66608000508583$

$-3.79128394116740$

$-4.52516817553505$

$-3.96088769752454$

$-3.01926284389810$

$-3.15350803436433$

$-1.90746362162723$

$-0.81439313198790$

1.14282965427839

1.68327953312752

1.86237478268839

0.56669975392560

1.38482579648834

0.10980210683765

$-1.72935716172374$

$-0.86668640060621$

$-2.61526448905637$

$-1.91888836458784$

$-2.27123698098086$

$-1.46153200039660$

$-3.11613451628181$

$-2.64312993214074$

$-2.99386963751153$

$-3.85878424122726$

$-3.37320063188002$

$-2.33885388952926$

$-0.77596893320451$

0.22889793063044

$-1.47880087677390$

$-1.10404765751843$

2.81347290212505

3.41966198650046

2.89555543175485

3.20059286998448

1.09586568908278

1.74872368252059

1.48102775141389

0.07044000097438

0.63652271788643

$-0.40949574087834$

0.74720645471494

1.30708174555133

0.37761492824209

$-0.64606154210553$

0.35761138924245

1.03111356475632

1.20594905181297

1.76918586690568

1.75336994120906

0.20965265396901

2.71194448149468

3.30575815133341

2.68668632656543

3.18538570414357

$-4.83558312197769$

$-5.11785267994572$

1.89460851824849
2.73360189651923

1.73805751486649

3.34765930305918

3.85549154951889

4.61159140200934

4.91288959174310

4.97755555286309

4.59237072212098

4.87168322958097

3.83685283154815

3.27846072024723

1.83408224878994

2.40148259150908

1.59901447081441

0.54862931845790

$-0.17732188927003$

0.76629642519735

$-0.90342334417781$

$-1.38339967467835$

$-1.54116528615268$

0.06181107948580

$-0.77349810793532$

$-0.54119651109871$

$-0.07537314627396$

$-1.79460486363238$

3.53886163314245

4.20894999843898

2.56660089465758

3.96955483157761

3.44116046127326

3.27048820795840

2.73031028976879

4.45925428090912

$-1.32667785724797$

$-2.25268927620630$

$-0.80727508156071$

$-0.67081212033232$

$-2.64899131201781$

$-3.53818037544690$

$-1.99603609936981$

$-2.97456118923356$

$-3.00689259030328$

$-3.32959222507548$

$-2.59710966007993$

$-3.87957897794239$

5.50310709477868

5.82130981908203

5.00450937555407

6.39443156718737

5.41541621964177

6.32921881761014

4.86455006403952

5.70459679904989

3.95969877726093

4.88784206332853

3.42777760636776

3.31404934730874

5.56810716116764

$-2.93000763292026$

1.27833219065452

$\left[\left({ }^{2} \mathrm{PDI}_{2}-\mathrm{H}_{2}\right) \mathrm{CO}_{2}\left(\mu-\mathrm{NH}_{2}\right)\left(\mathrm{PMe}_{3}\right)_{2}\right]^{3+}\left({ }^{2}\left[\mathrm{Co}_{2} \mathrm{NH}_{2}{ }^{*}\right]^{3+}, \mathrm{S}=0\right)$

$\mathrm{E}=-4923.83193827 E_{\mathrm{h}}$

Co $0.09102243099573 \quad-0.22543700375923 \quad 0.04587766233965$

Co $0.08509000108251 \quad-0.22228918963976 \quad 2.58672256387880$ 
N -0.09911370285363

P -0.11128025972098

P -0.13309783691373

N 2.01896908365998

N 0.34082585619496

N -1.73661648242700

N -1.74225669735918

N 0.34228584655547

N 2.01389896389799

C 2.57705513631241

C 1.60209272868013

C 1.79931053062444

H 2.80372685015963

C 0.68658045819406

C -0.60051847512269

H -1.46847922617512

C -0.75466228135054

C -1.96876331275300

C -2.82642100356173

H -3.80012667605562

H -2.68518732790000

C -2.84208463118931

H -2.73754600804288

H -3.81273576325255

C -1.96604091274123

C -0.74834098124490

C -0.58778454192159

H -1.45204375207559

C 0.70048418462968

C 1.80829103625691

H 2.81400320218340

C 1.60483909323119

C 2.57545712848678

C 2.79778791271832

H 3.38551947835250

H 0.67770525602080

C 2.80196222354026

H 3.39233111392827

C 4.05340048065861

H 4.40944785439409

H 4.33278455399732

H 4.59151421287550

C -3.32553322257880

H -3.92314752040059

H -3.89459997928716

H -3.24627902894436

C -3.31872721310045

H -3.23214048139081

H -3.88573571930883

H -3.92244190241434

C 4.05226338735568

H 4.40610667025443

H 4.58938212284241

H 4.33525243214934

C -0.43807617104162

H -0.51373581199795

H 0.38903939513415

H -1.38601647357928

C -1.45336449018460

H -1.45070439086126

H -2.42904339143911

H -1.29598015594455

C 1.36822792942490

H 1.64280671456230

H 2.21150051441086

H 1.15026532722300
1.17695194266994 1.02506117868837 1.03685632293865 $-0.23151142349250$ $-1.81780640106333$

$-0.79415372763618$ $-0.80351312078919$

$-1.80633409257572$

$-0.21685752232430$

$-1.21156003583837$

$-2.16736856619351$

$-3.33819267910472$

$-3.62847866739549$

$-4.13164138835574$

$-3.75328182205467$

$-4.37223152370014$

$-2.56896147642750$

$-1.94553489440093$

0.01226994123046

$-0.32385673320704$

1.04598791446060

$-0.01053182456529$

1.01648465860121

$-0.38579839774024$

$-1.95160528956469$

$-2.56269989344999$

$-3.74026706380955$

$-4.36336919865063$

$-4.10682068072272$

$-3.30812880926453$

$-3.58815083716947$

$-2.14483468050988$

$-1.18789863533177$

0.75129916654821

1.46658106623590

1.85176338938262

0.74236177645027

1.45060399470101

$-1.32312571775461$

$-0.51307411466768$

$-2.29064748073575$

$-1.20949092291163$

$-2.55569478641104$

$-1.97199759767327$

$-2.58839906864731$

$-3.58785020812184$

$-2.56822227705977$

$-3.59101112553463$

$-2.62735850728922$

$-1.97365713787634$

$-1.29080890732747$

$-0.46754837072049$

$-1.19280731661222$

$-2.24907943889916$

2.78559431878167

3.32751539049707

3.21080773732487

2.89969349353538

0.47478976884766

1.09262889740870

0.57886487444984

$-0.58382468671199$

1.01306468034366

$-0.02615265103075$

1.49777705233114

1.56949403832329
1.31431403697370

$-1.78773163787095$

4.41324389718081

$-0.01680060713157$

$-0.82020683073609$

$-0.00527228196613$

2.63880562756490

3.46490727161277

2.65272967517396

$-0.70741889216032$

$-1.20607322616492$

$-1.95513875170640$

$-2.28019647419595$

$-2.27832554966321$

$-1.85842408937309$

$-2.10723878765496$

$-1.12146131036320$

$-0.60483836869547$

0.53721689662507

0.15117712785054

0.18267773232022

2.09563714164335

2.48155071163358

2.45095128598418

3.24883710615457

3.77047923773606

4.51696151980706

4.76814142157683

4.94317512179927

4.61617127001199

4.94614120136732

3.85726782104490

3.35313292910538

1.98847551403278

2.57830684146770

1.31538789552555

0.63919169593243

0.04342808307424

$-0.91287698082133$

$-1.58288354769906$

$-1.36211755770160$

0.04850461171782

$-0.79043845138218$

$-1.52027584392694$

0.15750597929999

$-1.16925683821131$

3.44516523384913

3.84702210298944

2.49697185839691

4.16081165881455

3.56045302257125

4.21519306399224

2.59678688189881

4.02682294866852

$-1.46932988953768$

$-2.43170830604039$

$-0.87218139348443$

$-0.91324108521417$

$-2.88643390361524$

$-3.80472088777146$

$-2.38018301415365$

$-3.16265191493629$

$-2.84834758044149$

$-3.10548925669623$

$-2.32504472827260$

$-3.78006586133038$ 


$\begin{array}{llcc}\text { C } & -1.44338344488583 & 0.45383807788089 & 5.53334740449883 \\ \text { H } & -1.25472812381481 & -0.59939939628374 & 5.81035998425935 \\ \text { H } & -2.42840567296590 & 0.53068006706903 & 5.04052570358020 \\ \text { H } & -1.44420769179198 & 1.07480706756831 & 6.44948976700225 \\ \text { C } & 1.35859163997222 & 1.08143333397347 & 5.45533834362349 \\ \text { H } & 1.13753200540155 & 1.64705435809506 & 6.38078670438278 \\ \text { H } & 2.18219888075952 & 1.57893803786530 & 4.91316699203232 \\ \text { H } & 1.66302128856708 & 0.05385583509770 & 5.72572251292304 \\ \text { C } & -0.52445720307300 & 2.78186158972734 & 4.08303894551945 \\ \text { H } & -0.61591869552898 & 3.32855821344245 & 5.04135403477133 \\ \text { H } & -1.47908815512529 & 2.85505833534897 & 3.53130573818543 \\ \text { H } & 0.28276578417835 & 3.23293303496282 & 3.47797424052848 \\ \text { H } & 0.84043516765248 & -5.02006795277499 & 5.53259826928781 \\ \text { H } & 0.82177663273144 & -5.05038543270324 & -2.86028518512529 \\ \text { H } & -0.96717669792991 & 1.72175912170237 & 1.30833374183295\end{array}$

$\left[\left({ }^{2} \mathrm{PDI}_{2}-\mathrm{H}\right) \mathrm{Co}_{2}(\mu-\mathrm{NH})\left(\mathrm{PMe}_{3}\right)_{2}\right]^{3+}\left({ }^{2}\left[\mathrm{Co}_{2} \mathrm{NH}\right]^{3+}, \mathrm{S}=1,\left\langle\mathrm{~S}^{2}\right\rangle=2.021200\right)$

$\mathrm{E}=-4923.76622811 E_{\mathrm{h}}$

$\begin{array}{llll}\text { N } & -0.19094105771112 & 0.89029433135840 & 1.32337506574836\end{array}$

$\begin{array}{llll}\text { P } & -0.38728525048857 & 0.99091981125660 & -1.67058178508684\end{array}$

$\begin{array}{llll}\text { P } & -0.00405916903062 & 1.17709969266588 & 4.32771470561843\end{array}$

N $1.92116134080196 \quad-0.13102263339939 \quad-0.02431791978562$

N $0.45572655274909 \quad-1.84352996424120 \quad-0.98505501197479$

N $-1.78126774502911 \quad-1.24529221258130 \quad-0.02414032299123$

$\begin{array}{llll}\text { N } & -1.78486160407878 & -0.75243197981083 & 2.74995744658218\end{array}$

N $0.21433681828093 \quad-1.82661822895188 \quad 3.66351836282876$

N $2.01934989750128 \quad-0.42386644888523 \quad 2.71161641747776$

C $2.60523955445831 \quad-0.99682552567417 \quad-0.74217173760242$

C $1.75433534916163 \quad-2.00707327736343 \quad-1.35877125848784$

C $2.10798105187889-3.02902113133239 \quad-2.25143944466163$

H $3.15052919350306 \quad-3.17462739363402 \quad-2.55071290413887$

C $1.09242320741001 \quad-3.86080072074107 \quad-2.76351064091833$

$\begin{array}{llll}\text { C } & -0.23771744057009 & -3.66677726376253 & -2.38077353030877\end{array}$

H $-1.02853010550963 \quad-4.30722042214006 \quad-2.78376721306806$

C $-0.55398800525976 \quad-2.63267194607636 \quad-1.46553566204131$

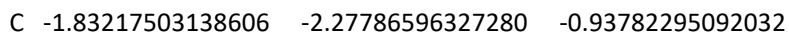

$\begin{array}{llll}\text { C } & -2.87605445161692 & -0.87452903413487 & 0.62701418978778\end{array}$

H $-3.85362952743583 \quad-1.26282747425050 \quad 0.31815961558061$

C $-2.79496314656171 \quad-0.09445839767292 \quad 1.90356813538325$

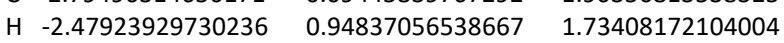

$\begin{array}{llll}\text { H } & -3.78536953901815 & -0.07754829322321 & 2.38566985157068\end{array}$

C $-2.09911930184941 \quad-1.80557176494443 \quad 3.47730915533793$

$\begin{array}{llll}\text { C } & -0.92955997910769 & -2.44360758605738 & 4.06977554749477\end{array}$

C $-0.85426221903853 \quad-3.56251883926960 \quad 4.91246687090630$

H $-1.76296283048523 \quad-4.07099022436602 \quad 5.25026865118472$

C $0.41114318875670 \quad-4.02401105760478 \quad 5.31248000598381$

$\begin{array}{llll}\text { C } 1.57446500421914 & -3.37419904567320 & 4.86896070249732\end{array}$

H $2.56044941849353 \quad-3.73612272196761 \quad 5.17686289965236$

C $1.44938665187581 \quad-2.25506773532689 \quad 4.02904941083643$

C $2.49020405279772 \quad-1.41293357689252 \quad 3.44846239622472$

$\begin{array}{llll}\text { C } 2.95817395230608 & 0.53291944051763 & 2.11790583696108\end{array}$

H $3.97015111817308 \quad 0.10052293120928 \quad 2.10206712235359$

H $3.00418804656886 \quad 1.43238994805870 \quad 2.76150519499302$

$\begin{array}{llll}\text { C } & 2.56979778943457 & 0.96376220760983 & 0.69385301814385\end{array}$

H $1.86076537031474 \quad 1.80201506565705 \quad 0.72667126627022$

H $3.47605743090240 \quad 1.30884051536056 \quad 0.16791754427298$

C $4.09046887059644 \quad-0.93974234406772 \quad-0.93215428250143$

H $4.37920852090448-0.02591568471621 \quad-1.49037460347585$

$\begin{array}{llll}\text { H } & 4.45647832106737 & -1.81354431665809 & -1.49555758175004\end{array}$

H $4.61882230776170 \quad-0.91606621734145 \quad 0.04005436077792$

$\begin{array}{llll}\text { C } & -3.10468384354451 & -2.98380634611775 & -1.26825478615487\end{array}$

H $-3.56123019205752 \quad-3.43038235691355-0.36001404356552$

H $-2.93671271729437 \quad-3.79552540816952-1.99468824280595$ 


\begin{tabular}{|c|c|c|c|}
\hline & -3.85033296 & $-2.28 / 42140 / 66 / 15$ & \\
\hline & & -2.340465 & \\
\hline & & & \\
\hline & & & \\
\hline & 00 & -1.5 & \\
\hline & 206 & 1.66 & \\
\hline & 3373 & -1.86 & \\
\hline & 369 & 05 & \\
\hline & 409 & -0.79 & \\
\hline & 33 & 2.7 & 777 \\
\hline & 83 & 3.25 & \\
\hline & .066 & 3.15 & \\
\hline & -1.690 & 2.72844 & -0.58 \\
\hline & 17 & 0.46 & -2 \\
\hline & 62 & & \\
\hline & -2.7 & & \\
\hline & & & -3. \\
\hline & 1 & .1 & 2.8 \\
\hline & 99 & & 77 \\
\hline & .87 & & 2. \\
\hline & 0.73 & & 5670 \\
\hline & -1.5 & 1.17 & 5.2 \\
\hline & -1.7 & 0.1 & 5.6 \\
\hline & -2.4 & 1.4 & \\
\hline & -1 & & \\
\hline & & 09 & 5.6 \\
\hline & 75 & 86 & 64 \\
\hline & $2 . \angle 0$ & & \\
\hline & $1.15<4$ & 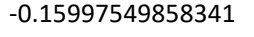 & \\
\hline & & & \\
\hline & 0 & 3.5 & 4.7 \\
\hline & -0.60400288104640 & 3.23306302796298 & $3.190386404 / 3 / /$ \\
\hline & 1.18417523328055 & & \\
\hline & 39 & 996 & 5.9 \\
\hline & & -4.6 & 3.4 \\
\hline & & & \\
\hline
\end{tabular}


C 2.93082902649208

H 2.37985925987686

H 3.75845880009418

C 0.82841143599171

C 0.11371193823725

C -1.18604938672734

H -1.80730808058298

C -1.67199578268318

C -0.86472004221686

H -1.24321180725543

C 0.43128560616213

C 1.43355101646058

C 3.60033879842892

C 5.43023041128058

C 8.19309765718044

H 8.13125861481243

H 9.25702739222005

H 7.69194838533144

C 6.80551698767842

H 5.98152108659325

H 7.75898281191345

H 6.75572020038005

C 0.17134769237882

H 0.14617466540233

H -0.87703207654158

H 0.67834979891593

C 1.14514819171470

H 1.28065135300455

H 0.11286263468132

H 1.83096678523704

C 5.40997090897762

H 5.89337623293589

H 4.85703908137383

H 4.69768036356754

C 7.89981614289046

H 8.31879311799295

H 8.71553497410834

H 7.44381286085068

C 7.56904985683411

H 6.87029067050948

H 8.36681116244524

H 8.02875829913256

C 2.90872143754146

H 3.98085753261656

H 2.74485309480867

H 2.60828182530281

C 0.19020153620507

H -0.04481192099244

H 0.07174790314086

H -0.50923465980246

C 1.88242218046244

H 1.44512341315224

H 1.26283494584225

H 2.90549707660043

C 5.04071232729679

H 10.88198389232218

H -2.68276574512911

H 5.86058356891337

H 4.53137475284002

H 5.21892739900494

H 5.69370527481786

H 3.56808913440875

H 3.29714438374117
11.48152510385021 10.82840236608082 10.89160770374082 12.31150427047436 12.70779830621849

13.22012269686418 13.37308038290685 13.53614345597931 13.32984393260168 13.56965313058845 12.81479388665291 12.49572820039565 11.62226080852811 10.36573945883002 9.31996284086795 9.72749866027541 9.15980869974368 8.33116543745210 13.54680698635026 13.25790081490480 13.30718730373943 14.64674419114930 12.43159848907646

13.49479661114220 12.08517070549719 11.85059979791613 12.63030576487658 11.66215088291122 12.97180110200832 13.35921367145905 15.04388187980881 15.95931578482293 14.54450790313476 15.31298941855878 13.60544064760691 14.56317929172272 12.99248679638833 13.06279443153741 14.99103297878432 15.39371915463105 14.41685776654381 15.83173646262853 8.54364283180876 8.66496474665976 8.80591472570144 7.49059629080393 9.39465478452863 8.31312272661823 9.78553376958487 9.91842276393575 8.83509641564956 7.82360747642764 9.42983243449764 8.74242295500019 11.58293199430490 10.64638050281134 13.94176404491185 9.56603932472034 9.95273737113724 12.50187092095779 11.62927019930790 12.33366190913442 10.63964038328755
6.10495836967984 5.40493004038592 6.52752148155512 6.98385622686595 8.19083150304258 8.30889137941846 7.42079944125040 9.58825292355079 10.71820321360399 11.71645669218198 10.54650455293104 11.55343799414272 12.05194000382442 10.70133681957645 10.47620957529749 11.50375588132591 10.23588105958543 10.47268056271733 4.46353833509310 3.78479643797624 3.96344386457379 4.59645683964770 5.64288269418344 5.32873593908352 5.70991979921963 4.85664576595579 13.01852568603583 13.53896259251299 13.19384173923815 13.49517346419602 9.91931567756988 10.31036715885909 10.73290603506917 9.11889291590384 10.52064824474331 10.88273720125537 10.09296209614589 11.36493983767815 8.03768393748296 7.28380312887848 7.53449570535858 8.59132850062085 8.13992166728942 8.37818261037794 7.07990957000282 8.30415770731021 8.61039093232875 8.60990310813546 7.58459358814753 9.28655799473459 10.83394414214307 10.73279007362541 11.52894134835208 11.23449890498169 11.55693503448914 6.13735196779977 9.70579642496349 11.32526841143297 10.21586427127034 10.98382821476395 12.44663770720409 12.89416769808584 12.46174630120855

$\operatorname{lin}-\left[\left({ }^{2} \mathrm{PDI}_{2}\right) \mathrm{Co}_{2}(\mu-\mathrm{N})\left(\mathrm{PMe}_{3}\right)_{2}\right]^{3+}\left(\operatorname{lin}^{2}{ }^{2}\left[\mathrm{CO}_{2} \mathrm{~N}\right]^{3+}, \mathrm{S}=0\right)$ 
$E=-4923.76657387 E_{\mathrm{h}}$

Co $5.68177778033849 \quad 11.59550512231129$

Co $2.64440854936644 \quad 11.35407170658221$

$\begin{array}{lll}\text { P } 5.90804338512625 & 13.73691371732503\end{array}$

$\begin{array}{lll}\text { P } & 1.57405685064768 & 9.39726115921389\end{array}$

N $6.31457839525626 \quad 10.74452967600553$

N $7.46942247944956 \quad 11.42599019580258$

N $5.51468628355818 \quad 12.23174184027856$

N $2.156777466663038 \quad 12.05691248486152$

N $1.17358950349394 \quad 12.35326436753591$

N $3.12577557221598 \quad 11.62378479832564$

N $4.15781153566608 \quad 11.06099337379648$

C $7.61747681516598 \quad 10.52194939691397$

C $8.33222545850772 \quad 10.94166497043907$

$\begin{array}{ll}\text { C } 9.69855774874614 & 10.88609332749867\end{array}$

H $10.43184080854516 \quad 10.51144225753898$

C $10.11289632633572 \quad 11.31860540362173$

C $9.18416902404579 \quad 11.79502777896829$

H $9.52128790620504 \quad 12.12031780474403$

C $7.82678022593920 \quad 11.84180178305470$

C $6.65255491997842 \quad 12.29493779100783$

C $4.23986414785807 \quad 12.73758727767169$

C $2.99641425977389 \quad 11.80358901473787$

H $2.40514120986648 \quad 11.93120488322562$

H $3.32562217775909 \quad 10.75084977430555$

C $0.99189881341462 \quad 12.68562903832132$

$\begin{array}{lll}\text { C } 0.40689868366859 & 12.90853850245047\end{array}$

C $-0.71331121100475 \quad 13.67219494696300$

H $-1.36173402359721 \quad 14.11508990949856$

C $-0.96907115382657 \quad 13.89305264422078$

C $-0.08727023390936 \quad 13.40526172031189$

H $-0.25620991750506 \quad 13.63897347602997$

C $1.02269861800188 \quad 12.64241963289092$

C $2.14539142866317 \quad 12.15761529489614$

C $4.36145500865211 \quad 11.20555052763086$

$\begin{array}{ll}\text { C } 5.35876707254270 & 10.23057786196817\end{array}$

C $8.32032594193566 \quad 9.86810443157210$

H 7.99853333813206

H 9.41240017635702

H 8.10327451375340

C 6.78296562208885

H 6.10368237737020

H 7.81310569143362

H 6.52848013385826

C 0.35781248507271

H 1.02219222976025

H -0.59703931616264

H 0.15071440878231

C 2.18933572466350

H 2.25859606026536

H 1.28866579179319

H 3.07620990392532

C 4.35625960694376

H 4.55616027180830

H 3.93839697733042

H 3.61997183010733

C 7.06070682142665

H 7.18709321111795

H 8.04185770979045

H 6.68359197672239

C 6.59812470524298

H 5.88010573675641

H 7.54529517318752

H 6.79882149989436

C 2.59581072543626

H 3.48920315937574
11.01031363682534

. 10.92485743382750

$8.78058595532710 \quad 11.02950139136796$

$12.81521990300735 \quad 4.29844340295936$

$12.27896190586734 \quad 3.60799985674225$

$12.69305322973735 \quad 3.92655219168664$

$13.89355320456788 \quad 4.25539460027013$

$13.17210500501141 \quad 5.79520232614377$

$13.88384097744222 \quad 5.26690019165309$

$13.68426393283748 \quad 5.99892733633688$

$12.32802935266487 \quad 5.10746078464823$

$12.30723313946576 \quad 12.98723944779747$

$11.31535789971350 \quad 13.47917547191926$

$12.81288453060239 \quad 13.37149840291037$

$12.89119165480907 \quad 13.30388212562113$

$\begin{array}{ll}14.52420898253994 & 9.52328609787389\end{array}$

$15.57981197310377 \quad 9.78887887315425$

$13.99510949977622 \quad 10.39502982016501$

$14.48311692644961 \quad 8.70110945631937$

$13.88139748217110 \quad 10.39532002436801$

$14.94915222496868 \quad 10.65764196915800$

$13.46339905329126 \quad 10.10369784504518$

$13.33108828359760 \quad 11.27431595801429$

$\begin{array}{ll}14.86818478659290 & 7.74755734538464\end{array}$

$\begin{array}{ll}15.00690381364574 & 6.92093340805922\end{array}$

$14.46112874191726 \quad 7.34925761465705$

$\begin{array}{ll}15.84827867006150 & 8.22103990558520\end{array}$

$8.02971545959835 \quad 8.28984479787512$

$7.91874887871503 \quad 8.93063840123869$ 

H 2.91956964884151
8.26128993168487
7.25872780646841
H 2.01308547496694
7.08831080958833
8.29672956073314
C 0.06647690690220
9.39253107925325
7.89971645185182
H -0.43721817964663
H 0.33299462979356
H -0.61991677927340
8.41034397863574
7.98582928540850
$9.57783397828053 \quad 6.84324567309318$
C 1.05024450874064
$10.18622677449673 \quad 8.24646987361886$
$\begin{array}{ll}8.92417337925717 & 10.58837750578981\end{array}$
H 0.48761721551592
7.97108526762386
10.55580313856287
$9.71601073330965 \quad 11.00988041104032$
H 0.40364138631263
H 1.94269179120369
8.80544034678885
11.22943858551429
H 11.17618755979465
11.27957735367850
6.78921630424981
H -1.83699801013931
14.49115006748365
H 4.01727853708162
H 4.37235961369000
13.69336415952968
12.96794574818011
10.69262672896122
4.07520166740
12.12381077354052
10.41347915125055
6.32668244439984
4.74761527868244
H 4.89533013524249
9.77708971346374
12.41927410039591
H 5.92100943787376
9.41357699460586
11.79415762835547
11.64606954891414
H 4.80176805195272
10.32029282807654

$\operatorname{lin}-\left[\left({ }^{2} \mathrm{PDI}_{2}\right) \mathrm{Co}_{2}(\mu-\mathrm{N})\left(\mathrm{PMe}_{3}\right)_{2}\right]^{3+}\left(\operatorname{lin}^{-2}\left[\mathrm{Co}_{2} \mathrm{~N}\right]^{3+}, \mathrm{S}=1,\left\langle\mathrm{~S}^{2}\right\rangle=2.026192\right)$

$\mathrm{E}=-4923.76627723 E_{\mathrm{h}}$

Co $5.58476256995534 \quad 11.42761373274783 \quad 8.18845152644092$

Co $2.83555196495776 \quad 11.18848666851994 \quad 8.84057097738557$

$\begin{array}{llll}\text { P } & 5.60011523736750 & 13.66017001434492 & 8.90348680495693\end{array}$

$\begin{array}{llll}P & 1.49713707242124 & 9.39709771476034 & 8.92147403378897\end{array}$

N $6.30823558863983 \quad 10.63506372718131 \quad 9.82099525929433$

N $7.43067136577305 \quad 11.54204381033031 \quad 7.75584626409215$

\begin{tabular}{llll} 
N 5.46924646808427 & 12.06373629807951 & 6.21858143581241 \\
\hline
\end{tabular}

$\begin{array}{llll}\text { N } & 2.28240840679454 & 11.96978967438807 & 7.07718145164834\end{array}$

$\begin{array}{llll}\text { N } & 1.45147737742157 & 12.39488319790739 & 9.40511255115309\end{array}$

N $3.29510362191929 \quad 11.37737578118530 \quad 10.83642434332119$

$\begin{array}{llll}\text { N } & 4.18796877903528 & 10.51254640501844 & 8.17298404535902\end{array}$

C $7.61757661843996 \quad 10.63289552202148 \quad 9.91604346065620$

C $8.30648721792513 \quad 11.15104474747316 \quad 8.71431674893564$

C $9.68317184353916 \quad 11.20963181507985 \quad 8.45024520300593$

H $10.41800776312199 \quad 10.91208312864867 \quad 9.20491499250879$

C $10.10414693629196 \quad 11.64739495565504 \quad 7.18188558970262$

$\begin{array}{llll}\text { C } & 9.16544470053394 & 12.01595409530362 & 6.20370262092309\end{array}$

H $9.50141264705863 \quad 12.34426798013213 \quad 5.21525717736226$

C $7.79766337060017 \quad 11.95152427071929 \quad 6.52122980484428$

C $6.63655529476070 \quad 12.30093765287096 \quad 5.67335875374363$

$\begin{array}{llll}\text { C } & 4.23233551946444 & 12.45560915689152 & 5.52871257700541\end{array}$

C $2.97495662524537 \quad 11.60895358276048 \quad 5.83525066512697$

H $2.29873142523557 \quad 11.72002616871093 \quad 4.97152548037775$

H $3.25834563792204 \quad 10.54554469234433 \quad 5.90574863277392$

C $1.22396121090830 \quad 12.74397835124034 \quad 7.09538198146092$

C $0.72392900220517 \quad 13.02862901924376 \quad 8.45270004711449$

$\begin{array}{llll}\text { C } & -0.30729647443188 & 13.90046150694887 & 8.83762507707828\end{array}$

H $-0.91847725955696 \quad 14.41842252375919 \quad 8.09191619930220$

C $-0.52935703006484 \quad 14.11738531067436 \quad 10.20795473639512$

C $0.29556636230102 \quad 13.50665681783005 \quad 11.16676211713643$

H $0.15229915508916 \quad 13.71800145256890 \quad 12.23081456151119$

C $1.31592901972412 \quad 12.64512817803610 \quad 10.72923061247465$

C $2.37850260333251 \quad 12.01582030276318 \quad 11.53260289989611$

C $4.42785873151908 \quad 10.78669381669865 \quad 11.56710127516682$

C $5.43778595815253 \quad 9.94315742653622 \quad 10.78069251918280$

$\begin{array}{lll}\text { C } 8.38796818713237 & 10.09780139283538 & 11.08537733609432\end{array}$

H $7.92211491642325 \quad 10.38867475123138 \quad 12.04410618849337$

H $9.42480427903002 \quad 10.47323250447815 \quad 11.07964090769017$

H $8.42919779171406 \quad 8.98956932648064 \quad 11.04528558259437$

C $6.82158199334514 \quad 12.92507043597639 \quad 4.32183269329885$

H $6.34146995874158 \quad 12.30928306636154 \quad 3.53546627078976$

H $7.88617362897989 \quad 13.04011554031357 \quad 4.06372235410058$

H $6.34907304893397 \quad 13.92697019447441 \quad 4.29137952659909$

C $0.58286193729146 \quad 13.32442569287669 \quad 5.86984677534674$ 


\begin{tabular}{|c|c|c|c|}
\hline $\mathrm{H}$ & 1.31351949865571 & 13.90980380608184 & 5.27910693087339 \\
\hline & -0.25849091597119 & 13.98723077945465 & 5848 \\
\hline & 0.19428762302339 & 12.51686719101044 & 5.21710218105783 \\
\hline & 2.41920826167888 & 12.14688639478796 & 13.02347925387032 \\
\hline & 2.42138903290021 & 11.14564224899918 & 13.49949859183196 \\
\hline & 1.55935532753340 & 12.71266794744282 & 13.41532025785179 \\
\hline & 3.34854376464895 & 12.65661240932958 & 13.34791037218494 \\
\hline & 4.01831171644292 & 14.51770574511470 & 5988102077 \\
\hline & 4.20944586224388 & 15.59031487113541 & 9.38325688779630 \\
\hline & 3.51340819352024 & 14.07608422519093 & 10.06333822359736 \\
\hline & 3.37153323585951 & 14.41337658948892 & 8.30135095326973 \\
\hline & 6.52249700164157 & 71143675429 & 83064511828 \\
\hline & 6.5986174 & 14.9 & 22171626755 \\
\hline & 7.54000788224489 & 13.44903660008739 & 21293443804 \\
\hline 7 & 6.00678711637057 & 13.33728678786665 & 11.28099983858663 \\
\hline - & 6.46905583135429 & 14.77636648443541 & 8450887546 \\
\hline 7 & 5.92107151250301 & 14.84 & 6177877478 \\
\hline & 7.49 & 14.4 & 347 \\
\hline 1 & 6.52326647257655 & 15.78452391072059 & 8.20360833443696 \\
\hline - & 2.31517132934617 & 7.87490618717989 & 2951993904 \\
\hline $\mathrm{H}$ & 3.18266033827775 & 7.66 & 9951199670 \\
\hline 1 & 43067382462 & 8.014 & 7.32 \\
\hline & 1.60237144453645 & 7.02880688 & 1312290708 \\
\hline$C$ & 0.02841295307339 & 9.58026197235722 & 7.86619657131839 \\
\hline $\mathrm{H}$ & -0.60826836789589 & 8.67893863563611 & 7.95730960215942 \\
\hline $\boldsymbol{H}$ & 0.34425199484923 & 9.70083986756073 & 6.81422928008652 \\
\hline $\mathrm{H}$ & -0.55245490172069 & 10.46723897396177 & 8.17841472360521 \\
\hline - & 0.86331776904772 & 9.04642785568451 & 10.58845022579619 \\
\hline$H$ & 0.18444172160034 & 8.17249902865880 & 10.55116254648661 \\
\hline $\mathrm{H}$ & 0.30540217464595 & 9.92162598264028 & 10.96835327576133 \\
\hline H & 1.70763842088874 & 8.82659576392579 & 11.26682627796728 \\
\hline 7 & 11.17466746698171 & 11.69325045108635 & 6.95120110537478 \\
\hline $\mathrm{H}$ & -1.32982974450494 & 14.79342066154366 & 10.52961060914624 \\
\hline דו & 4.02789874086146 & 13.51939152320356 & 5.76735656188816 \\
\hline $\mathrm{H}$ & 4.38442837832088 & 12.39530813584861 & 4.43739757683349 \\
\hline $\mathrm{H}$ & 4.03364455921464 & 10.10832130456287 & 12.34949402975087 \\
\hline 7 & 4.95957058708249 & 11.59476579438240 & 12.10611652356848 \\
\hline 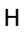 & 6.04395395608632 & 9.41789502222624 & 11.53629633352674 \\
\hline & 4.90041153369427 & 9.16758395359207 & 10.20432183191450 \\
\hline
\end{tabular}

\begin{tabular}{|c|c|c|}
\hline \multicolumn{3}{|c|}{$\left[\left({ }^{3} \mathrm{PDI}_{2}-\mathrm{RF}\right) \mathrm{Co}_{2}(\mu-\mathrm{N})\left(\mathrm{PMe}_{3}\right)_{2}\right]^{3+}\left({ }^{3}\left[\mathrm{Co}_{2} \mathbf{N}-\mathbf{R F}\right]^{3+}, \mathrm{S}=0\right)$} \\
\hline 927609 & 13.80196863692924 & 8.45649820520681 \\
\hline Co 2.843 & 15.17035972507605 & 6.42587518959553 \\
\hline P 2.71916966532520 & 17.20428933647177 & 5.42386461472365 \\
\hline P 2.85331146860610 & 13.60659795139072 & 10.72374391163959 \\
\hline N 4.78834005837306 & 15.10760386032690 & 6.09520445041877 \\
\hline N 2.91899817366520 & 11.9951635138 & 7.853 \\
\hline N 0.96614 & 13.423016345 & 8.418 \\
\hline N 0.9262 & 14.91776898220 & 6.11035575421848 \\
\hline C 3.01318170222370 & 11.85947982504594 & 3.17710078362676 \\
\hline N 4.82617 & 13.4728985028 & 8.44380804 \\
\hline C 1.73675 & 10.16390 & 6.88786795402221 \\
\hline H 0.7985 & 9.66292 & 6.629023650 \\
\hline N 2.9072 & 13.95239138886223 & 4.95840002154802 \\
\hline C 1.74004 & 11.40491774621475 & 7.54722115969317 \\
\hline C 4.11550 & 11.44145293168076 & 7.56011971532486 \\
\hline C 1.7689 & 12.3368263842 & 3.62347519494761 \\
\hline H $\quad 0.8427$ & 11.8736 & 3.26880207322175 \\
\hline C 2.9679 & 9.57253723947861 & 6.55626182224666 \\
\hline C 5.73343719578906 & 15.94869790399688 & 6.84594249092014 \\
\hline H 6.39577863860941 & 16.48089075735651 & 6.14026859336171 \\
\hline H 5.13930831878245 & 16.69551452157968 & 7.39554264775869 \\
\hline C 4.11461 & 13.52631426040943 & 4.52843670092275 \\
\hline C -0.02698012756549 & 14.37160019186825 & 8.94015165402011 \\
\hline
\end{tabular}


H 0.11758566792431

H -1.04535949001727

C -0.10655178228678

H -0.08681925666680

H -1.10497711619139

C 4.17561925205507

H 5.13775781104029

C 1.74365858538259

C 0.59779676633824

C 5.19441322050993

C 4.20410438758988

H 5.17680579421011

C 5.78545962710685

H 5.20655115171302

H 6.48306740680965

C 1.64234413493833

H 0.60956951980039

H 1.71405895702419

H 1.89431701506277

C 2.48929196913337

H 3.28674871151409

H 2.46007379991102

H 1.52246784667733

C 5.21563127611040

C 6.60680710055937

H 7.25757021568375

H 6.62213046031848

H 7.05032153608867

C -0.80445323161845

H -1.47383276395440

H -0.82582612226420

H -1.23632136918990

C 0.61266948076434

C 4.38691236418064

H 4.74382381081973

H 4.16356212158642

H 5.16708996351609

C 6.62459215462884

H 6.98610399685850

H 6.65731463887444

H 7.32877079879401

C -0.79366255874750

H -1.50686159353922

H -1.13662304013562

H -0.85709683492322

C 4.23630537589582

H 5.00527597723270

H 3.98462247069966

H 4.62334354101545

C 2.25475876823871

H 1.27919334927783

H 2.19917889592199

H 3.02337647053911

C 1.53025450548012

H 1.82636551544150

H 1.56836308886478

H 0.49894006215130

C 0.08770858179854

C 6.58585103378791

H 7.27424382753774

H 7.21812721610843

N 3.01613984243786

H 2.98577790289632

H 3.05397446060454

H -0.70122477968088

H 1.05745586414369
14.45704591011791 13.97861313589162 15.70520173034641 16.73109820348478 15.29666914427537 10.20527685801900 9.74406537256927 13.40664744189190 14.06875435017421 14.29901264289347 12.46314002423347 12.10674990008582 14.47143001863962 15.23002374455909 13.99242161787707 12.36786340588016 12.64069803546254 12.27694329561392 11.39458444084632 15.16862483924733 15.89394920196770 14.99910314155124 15.58071883686844 12.29755462439535 14.13466255194182 14.97623039375273 14.03932853371644 13.20414409045691 13.81415081610051 13.49705844173869 13.03124303675817 14.73607140559669 12.23344678069233 13.01040435916543 12.09494441723813 12.77784439200649 13.78720015927464 11.78878822338718 11.72948380198042 10.75987346736080 12.41242910590163 11.71154213702816 12.43391150926569 11.49158327000770 10.77624324540196 17.80270729675589 18.05708816790786 18.71306443412094 17.03184883327377 18.55212617093462 18.34757354225575 19.50578308657179 18.62781151815457 17.21677385579816 16.43379642789446 18.20401281313434 17.02725921162229 15.75202073417710 15.15502917731352 15.87078906730956 14.42204379686625 15.48353030201442 8.61046030164440 11.01750028128990 16.39457667179513 16.22123522212712
10.03103066958111 8.79064748707843 6.79931394773952 6.38892912790999 6.57523250232908 6.90031290605055 6.65389480480430 4.53424034425060 5.15861022142739 5.14569618386585 3.61754554004738 3.26257485176447 8.94358024349854 9.49250529308309 9.65278268536303 11.29583104769491 11.02587285664635 12.39655372658880 10.83472201246846 11.58278666148721 11.33775043641897 12.67661556595656 11.24658119417646 8.01131452305785 4.67161671365348 4.95818067141005 3.56993990620105 5.08112617486789 4.68846510145222 5.50998512847007 3.91324800506891 4.25012389704380 7.97856732859691 11.50932279714797 11.00475304800215 12.56818540438344 11.47554849932837 7.97340278801050 6.92683368154772 8.37968275342761 8.54508741896089 7.95172759465351 7.51608061214003 8.98351711077501

7.37219013277149 4.61051884575725 5.35713637654745 4.03325707137169 3.92050342200391 6.55355914546280 7.02701910430316 5.99365313522019 7.34462653184069 4.03995686476246 3.31718988088041 3.54136538171162 4.37876242159392 8.30718755069633 7.83567601368400 8.32452440991626 7.30441175794198 8.11197751283906 6.03175772573175 2.47681927285870 8.73945495452134 8.56624027085834 
$\mathrm{PMe}_{3}(\mathrm{~S}=0)$

$\mathrm{E}=-460.86033745 E_{\mathrm{h}}$

$\begin{array}{llll}\text { P } & 6.44630184126779 & 13.82298499902034 & 9.19017647970709\end{array}$

C $5.27110317588477 \quad 15.07209422798065 \quad 9.88922720193752$

H $5.80785053158865 \quad 15.95041753756186 \quad 10.30378471922705$

H $4.6703317447818214 .60398569043401 \quad 10.69380300239043$

H $4.58035485216479 \quad 15.41403427476438 \quad 9.09335976101111$

C $7.60819313826600 \quad 13.64251538771693 \quad 10.62082188684791$

H $7.97481945704910 \quad 14.62848113560106 \quad 10.97371623724719$

H $8.47505284437229 \quad 13.02232870286618 \quad 10.31821665137314$

H $7.08908186969099 \quad 13.13097006890056 \quad 11.45536175559610$

C $7.45982701730107 \quad 14.91614790897009 \quad 8.09184032659977$

H $6.84852624880498 \quad 15.23906444807600 \quad 7.22646140270951$

H $8.33222612338886 \quad 14.35065128788021 \quad 7.70981578393423$

H $7.82079115543888 \quad 15.81419433022774 \quad 8.63434479141893$

$\mathrm{N}_{2}(\mathrm{~S}=0)$

$\mathrm{E}=-109.51784399 E_{\mathrm{h}}$

$\begin{array}{llll}\text { N } & 0.00000000000000 & 0.00000000000000 & -0.00426439985789\end{array}$

$\begin{array}{llll}\mathrm{N} & 0.00000000000000 & 0.00000000000000 & 1.09426439985789\end{array}$

$\mathrm{H}_{2}(\mathrm{~S}=0)$

$\mathrm{E}=-1.17786675 E_{\mathrm{h}}$

H $\quad 0.000000 \quad 0.000000 \quad 0.116861$

$\begin{array}{llll}H & 0.000000 & 0.000000 & 0.883139\end{array}$ 